- Supporting Information for

\title{
Palladium-Catalyzed Three-Component Silylalkoxylation of 1,3-Diene with Alcohol and Disilane via Oxidative Coupling
}

\author{
Kazuyuki Torii, Kazuki Tabaru, Yasushi Obora* \\ Department of Chemistry and Materials Engineering, Faculty of Chemistry, \\ Materials and Bioengineering, Kansai University, Suita, Osaka 564-8680 Japan
}

Table of Contents:

S2: $\quad$ General Experimental Details

S2: $\quad$ Experimental Procedures

S3: $\quad$ Product Characterization

S10: $\quad$ Mechanistic Studies

S13: $\quad$ Time Course

S14: References

S15: NMR Data 


\section{General Experimental Details}

GLC analysis was performed using a Shimadzu GC-2010 with a flame ionization detector and a $0.22 \mathrm{~mm} \times 25 \mathrm{~m}$ capillary column (BP-5). NMR spectra were acquired at $400 \mathrm{MHz}\left({ }^{1} \mathrm{H} \mathrm{NMR}\right), 100$ $\mathrm{MHz}\left({ }^{13} \mathrm{C} \mathrm{NMR}\right)$, and $79 \mathrm{MHz}\left({ }^{29} \mathrm{Si} \mathrm{NMR}\right)$ respectively, in $\mathrm{CDCl}_{3}$ or $\mathrm{C}_{6} \mathrm{D}_{6}$ with a JEOL JNM-ECZ400S. Chemical shifts are reported in ppm relative to the trimethylsilyl (TMS) group in each product or hexamethyldisilane. The products were characterized by ${ }^{1} \mathrm{H} \mathrm{NMR},{ }^{13} \mathrm{C} \mathrm{NMR}$, gas chromatographymass spectrometry (GC-MS, EI ionization), infrared spectroscopy (IR), and High-resolution mass spectra (HR-MS, EI-TOF). GC-MS spectra were recorded on a Shimadzu GCMS-QP2010 SE. IR spectrum were obtained on a FT-IR (Shimadzu IRAffinity-1). HR-MS were recorded on a JEOL JMS-T100GCV with TOF mass analyzer at the Global Facility Center, Hokkaido University. The reagents, 2,3-dibuthyl-1,3-butadiene ${ }^{[1]}$ and 2,3-diphenyl-1,3-butadiene ${ }^{[2]}$ were prepared according to literature procedures. $\mathrm{Pd}(\mathrm{dba})_{2}$ was purchased from Sigma-Aldrich at reagent grade. Other reagents were purchased from commercial suppliers (TCI, FUJIFILM Wako Pure Chemical Corporation, Sigma-Aldrich, and Alfa Aesar Chemical Company) at reagent grade or higher purity (typically $>95 \%$ ) and used without further purification. We used $\mathrm{O}_{2}$ gas from gas cylinder at 99.9 vol. \%

\section{Experimental Procedures}

Typical reaction and product isolation for palladium-catalyzed three-component silylalkoxylation of 1,3-diene with alcohol and disilane via oxidative coupling (Table 1, entry 1): $\operatorname{Pd}(\mathrm{dba})_{2}\left(29 \mathrm{mg}, 5 \mathrm{~mol} \%\right.$; dba = (E, E)-dibenzylideneacetone), $\mathrm{CuBr}_{2}$ (22 mg, $\left.10 \mathrm{~mol} \%\right)$, and 1,4benzoquinone (43 mg, $40 \mathrm{~mol} \%$ ) were stirred in $N, N$-dimethylformamide (DMF; $3 \mathrm{~mL}$ ) for $30 \mathrm{~min}$ at room temperature. Then, 2,3-dimethyl-1,3-butadiene (1a; $246 \mathrm{mg}, 3 \mathrm{mmol}$ ), benzyl alcohol (2a; $108 \mathrm{mg}, 1 \mathrm{mmol})$, and hexamethyldisilane (3a; $585 \mathrm{mg}, 4 \mathrm{mmol})$ were added to the solution. The reaction mixture was stirred for $16 \mathrm{~h}$ at $70{ }^{\circ} \mathrm{C}$ (oil bath temperature) under $\mathrm{O}_{2}$ (balloon). Then, the reaction was quenched with toluene $(10 \mathrm{~mL})$. The product yields were estimated following GC analysis using an internal standard (decane). The product (4a) was isolated by filtration, evaporation in vacuo, and silica gel column chromatography (using hexane as the eluent) and obtained in $75 \%$ yield (197 mg) as a colorless liquid.

\section{Multi-gram scale synthesis of 4 a}

$\operatorname{Pd}(\mathrm{dba})_{2}(58 \mathrm{mg}, 1 \mathrm{~mol} \%), \mathrm{CuBr}_{2}$ (44 mg, $2 \mathrm{~mol} \%$ ), and 1,4-benzoquinone (1.30 g, 1.2 equiv.) were stirred in DMF (10 mL) for $30 \mathrm{~min}$ at room temperature. Then, 2,3-dimethyl-1,3-butadiene (1a; 2.48 g, $30.1 \mathrm{mmol})$, benzyl alcohol (2a; $1.12 \mathrm{~g}, 10.4 \mathrm{mmol})$, and hexamethyldisilane (3a; $5.85 \mathrm{~g}, 40.0$ 
mmol) were added to the solution, and the reaction mixture was stirred for $16 \mathrm{~h}$ at $70{ }^{\circ} \mathrm{C}$ (oil bath temperature) under $\mathrm{O}_{2}$ (balloon). The resulting mixture was filtered, and the solvent was evaporated in vacuo. The solution was extracted using hexane $(20 \times 5 \mathrm{~mL})$ and DMF $(20 \mathrm{~mL})$. The combined organic layer was evaporated in vacuo. Then, the solution was purified by silica gel column chromatography (using hexane as the eluent) to obtain $\mathbf{4 a}$ in $67 \%$ yield (1.84 g) as a pale orange liquid.

\section{Product Characterization}

4a: The reaction and purification were performed using the typical reaction and isolation procedure; yield 75\% (197 mg), colorless liquid; IR (neat): 2952, 2857, 1419, 1248, 1164, 1066, 847, 733, 697 $\mathrm{cm}^{-1} ;{ }^{1} \mathrm{H}$ NMR (400 MHz; $\left.\mathrm{CDCl}_{3}\right) \delta: 7.40-7.29(\mathrm{~m}, 5 \mathrm{H}), 4.49$ (s, 2H), 3.94 (s, 2H), 1.78 (dd, $J=1.8$, $0.9 \mathrm{~Hz}, 3 \mathrm{H}), 1.71(\mathrm{dd}, J=1.7,0.8 \mathrm{~Hz}, 3 \mathrm{H}), 1.62(\mathrm{~d}, J=0.9 \mathrm{~Hz}, 2 \mathrm{H}), 0.00(\mathrm{~s}, 9 \mathrm{H}) ;{ }^{13} \mathrm{C} \mathrm{NMR}(100$ $\left.\mathrm{MHz} ; \mathrm{CDCl}_{3}\right) \delta: 139.8(\mathrm{C}), 132.8(\mathrm{C}), 129.2(2 \mathrm{CH}), 128.8(2 \mathrm{CH}), 128.3(\mathrm{CH}), 123.1(\mathrm{C}), 72.8\left(\mathrm{CH}_{2}\right)$, $71.9\left(\mathrm{CH}_{2}\right), 26.5\left(\mathrm{CH}_{2}\right), 22.6\left(\mathrm{CH}_{3}\right), 17.5\left(\mathrm{CH}_{3}\right), 0.0\left(3 \mathrm{CH}_{3}\right)$; GC-MS (EI) $\mathrm{m} / z$ (relative intensity) 262 (1) $[\mathrm{M}]^{+}, 73$ (100), 91 (64), 82 (59); HR-MS (EI) $\mathrm{m} / z$ calcd for $\mathrm{C}_{16} \mathrm{H}_{26} \mathrm{OSi}[\mathrm{M}]^{+}: 262.1753$, found 262.1751 .

4a- $d_{7}$ : The reaction and purification were performed using the typical reaction and isolation procedure; yield 65\% (175 mg), colorless liquid; IR (neat): 2952, 2858, 1414, 1249, 1163, 1064, 969, 845, 762, $690 \mathrm{~cm}^{-1} ;{ }^{1} \mathrm{H} \mathrm{NMR}\left(400 \mathrm{MHz} ; \mathrm{CDCl}_{3}\right) \delta: 3.94(\mathrm{~s}, 2 \mathrm{H}), 1.78(\mathrm{~d}, J=0.9 \mathrm{~Hz}, 3 \mathrm{H}), 1.71(\mathrm{~d}, J$ $=0.8 \mathrm{~Hz}, 3 \mathrm{H}), 1.62(\mathrm{~d}, J=0.8 \mathrm{~Hz}, 2 \mathrm{H}), 0.00(\mathrm{~s}, 9 \mathrm{H}) ;{ }^{13} \mathrm{C} \mathrm{NMR}\left(100 \mathrm{MHz} ; \mathrm{CDCl}_{3}\right) \delta: 139.5(\mathrm{C}), 132.8$ (C), 128.7-127.9 (m, 5CD), $123.1(\mathrm{C}), 71.8\left(\mathrm{CH}_{2}\right), 26.5\left(\mathrm{CH}_{2}\right), 22.6\left(\mathrm{CH}_{3}\right), 17.5\left(\mathrm{CH}_{3}\right), 0.0\left(3 \mathrm{CH}_{3}\right)$; GC-MS (EI) $m / z$ (relative intensity) 269 (1) [M] $]^{+}, 73$ (100), 82 (63), 98 (61); HR-MS (EI) $\mathrm{m} / z$ calcd for $\mathrm{C}_{16} \mathrm{H}_{19} \mathrm{D}_{7} \mathrm{OSi}[\mathrm{M}]^{+}:$269.2192, found 269.2183.

4b: The reaction and purification were performed using the typical reaction and isolation procedure; yield 75\% (207 mg), colorless liquid; IR (neat): 2953, 2857, 1456, 1349, 1248, 1164, 1066, 845, 742, $692 \mathrm{~cm}^{-1} ;{ }^{1} \mathrm{H}$ NMR $\left(400 \mathrm{MHz} ; \mathrm{CDCl}_{3}\right) \delta: 7.35(\mathrm{~d}, J=7.1 \mathrm{~Hz}, 1 \mathrm{H}), 7.22-7.16(\mathrm{~m}, 3 \mathrm{H}), 4.47$ (s, 2H), 3.95 (s, 2H), 2.37 (s, 3H), 1.76 (s, 3H), 1.70 (s, 3H), 1.63 (s, 2H), 0.00 (s, 9H); ${ }^{3} \mathrm{C}$ NMR (100 MHz; $\left.\mathrm{CDCl}_{3}\right) \delta$ : $137.6(\mathrm{C}), 137.6(\mathrm{C}), 132.6(\mathrm{C}), 131.0(\mathrm{CH}), 129.7(\mathrm{CH}), 128.5(\mathrm{CH}), 126.6(\mathrm{CH}), 123.2$ (C), $72.1\left(\mathrm{CH}_{2}\right), 71.2\left(\mathrm{CH}_{2}\right), 26.5\left(\mathrm{CH}_{2}\right), 22.6\left(\mathrm{CH}_{3}\right), 19.7\left(\mathrm{CH}_{3}\right), 17.6\left(\mathrm{CH}_{3}\right), 0.0\left(3 \mathrm{CH}_{3}\right) ; \mathrm{GC}-\mathrm{MS}$ (EI) $m / z$ (relative intensity) $276(1)[\mathrm{M}]^{+}, 73$ (100), 105 (74), 104 (49); HR-MS (EI) $\mathrm{m} / z$ calcd for $\mathrm{C}_{17} \mathrm{H}_{28} \mathrm{OSi}[\mathrm{M}]^{+}:$276.1909, found 276.1910. 
4c: The reaction and purification were performed using the typical reaction and isolation procedure; yield 74\% (204 mg), colorless liquid; IR (neat): 2953, 2856, 1458, 1247, 1163, 1064, 845, 775, 740, $694 \mathrm{~cm}^{-1} ;{ }^{1} \mathrm{H}$ NMR (400 MHz; $\left.\mathrm{CDCl}_{3}\right) \delta: 7.29-7.11(\mathrm{~m}, 4 \mathrm{H}), 4.45$ (s, 2H), $3.93(\mathrm{~s}, 2 \mathrm{H}), 2.38$ (s, 3H), $1.77(\mathrm{~d}, J=0.8 \mathrm{~Hz}, 3 \mathrm{H}), 1.71(\mathrm{~d}, J=0.8 \mathrm{~Hz}, 3 \mathrm{H}), 1.62(\mathrm{~d}, J=0.7 \mathrm{~Hz}, 2 \mathrm{H}), 0.00(\mathrm{~s}, 9 \mathrm{H}) ;{ }^{13} \mathrm{C}$ NMR

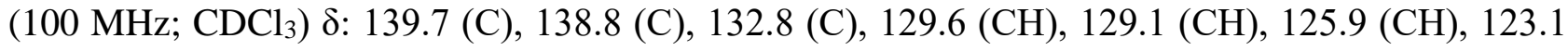
(C), $72.8\left(\mathrm{CH}_{2}\right), 71.9\left(\mathrm{CH}_{2}\right), 26.5\left(\mathrm{CH}_{2}\right), 22.6\left(\mathrm{CH}_{3}\right), 22.3\left(\mathrm{CH}_{3}\right), 17.6\left(\mathrm{CH}_{3}\right), 0.0\left(3 \mathrm{CH}_{3}\right)$; GC-MS (EI) $m / z$ (relative intensity) $276(1)[\mathrm{M}]^{+}, 73$ (100), 105 (72), 179 (42); HR-MS (EI) $\mathrm{m} / z$ calcd for $\mathrm{C}_{17} \mathrm{H}_{28} \mathrm{OSi}[\mathrm{M}]^{+}:$276.1909, found 276.1911.

4d: The reaction and purification were performed using the typical reaction and isolation procedure; yield 76\% (210 mg), pale yellow liquid; IR (neat): 2954, 2856, 1458, 1375, 1246, 1163, 1082, 844, 805, 754, $692 \mathrm{~cm}^{-1} ;{ }^{1} \mathrm{H}$ NMR $\left(400 \mathrm{MHz} ; \mathrm{CDCl}_{3}\right) \delta: 7.28(\mathrm{~d}, J=8.7 \mathrm{~Hz}, 2 \mathrm{H}), 7.18(\mathrm{~d}, J=7.9 \mathrm{~Hz}, 2 \mathrm{H})$, $4.45(\mathrm{~s}, 2 \mathrm{H}), 3.92(\mathrm{~s}, 2 \mathrm{H}), 2.38(\mathrm{~s}, 3 \mathrm{H}), 1.77(\mathrm{~s}, 3 \mathrm{H}), 1.71(\mathrm{~s}, 3 \mathrm{H}), 1.62(\mathrm{~s}, 2 \mathrm{H}), 0.00(\mathrm{~s}, 9 \mathrm{H}) ;{ }^{13} \mathrm{C} \mathrm{NMR}$ $\left(100 \mathrm{MHz} ; \mathrm{CDCl}_{3}\right)$ 8: $138.0(\mathrm{C}), 136.7(\mathrm{C}), 132.7(\mathrm{C}), 129.9(2 \mathrm{CH}), 128.9(2 \mathrm{CH}), 123.2(\mathrm{C}), 72.6$ $\left(\mathrm{CH}_{2}\right), 71.7\left(\mathrm{CH}_{2}\right), 26.5\left(\mathrm{CH}_{2}\right), 22.6\left(\mathrm{CH}_{3}\right), 22.1\left(\mathrm{CH}_{3}\right), 17.6\left(\mathrm{CH}_{3}\right), 0.0\left(3 \mathrm{CH}_{3}\right) ; \mathrm{GC}-\mathrm{MS}(\mathrm{EI}) \mathrm{m} / \mathrm{z}$ (relative intensity) 276 (1) $[\mathrm{M}]^{+}, 73$ (100), 105 (74), 179 (37); HR-MS (EI) $m / z$ calcd for $\mathrm{C}_{17} \mathrm{H}_{28} \mathrm{OSi}$ $[\mathrm{M}]^{+}:$276.1909, found 276.1908.

4e: The reaction and purification were performed using the typical reaction and isolation procedure; yield 75\% (239 mg), colorless liquid; IR (neat): 2955, 2861, 1360, 1248, 1164, 1064, 847, 764, 691 $\mathrm{cm}^{-1} ;{ }^{1} \mathrm{H}$ NMR $\left(400 \mathrm{MHz} ; \mathrm{CDCl}_{3}\right) \delta: 7.43-7.30(\mathrm{~m}, 4 \mathrm{H}), 4.47(\mathrm{~s}, 2 \mathrm{H}), 3.93(\mathrm{~s}, 2 \mathrm{H}), 1.79(\mathrm{~d}, J=0.9$ $\mathrm{Hz}, 3 \mathrm{H}), 1.71(\mathrm{~d}, J=0.6 \mathrm{~Hz}, 3 \mathrm{H}), 1.62(\mathrm{~d}, J=0.8 \mathrm{~Hz}, 2 \mathrm{H}), 1.36(\mathrm{~s}, 9 \mathrm{H}), 0.00(\mathrm{~s}, 9 \mathrm{H}) ;{ }^{13} \mathrm{C}$ NMR $(100$ $\left.\mathrm{MHz} ; \mathrm{CDCl}_{3}\right) \delta: 151.3(\mathrm{C}), 136.7(\mathrm{C}), 132.7(\mathrm{C}), 128.7(2 \mathrm{CH}), 126.1(2 \mathrm{CH}), 123.1(\mathrm{C}), 72.6\left(\mathrm{CH}_{2}\right)$, $71.8\left(\mathrm{CH}_{2}\right), 35.4(\mathrm{C}), 32.3\left(\mathrm{CH}_{3}\right), 26.4\left(\mathrm{CH}_{2}\right), 22.6\left(\mathrm{CH}_{3}\right), 17.6\left(\mathrm{CH}_{3}\right), 0.0\left(3 \mathrm{CH}_{3}\right)$; GC-MS (EI) $\mathrm{m} / z$ (relative intensity) 318 (1) $[\mathrm{M}]^{+}, 73$ (100), 147 (40), 179 (132); HR-MS (EI) $m / z$ calcd for $\mathrm{C}_{20} \mathrm{H}_{34} \mathrm{OSi}$ $[\mathrm{M}]^{+}:$318.2379, found 318.2376.

4f: The reaction was performed using the typical reaction procedure. The resulting mixture was filtered and evaporated in vacuo. The solution was extracted with hexane $(20 \times 5 \mathrm{~mL})$ and DMF $(20$ $\mathrm{mL}$ ). The combined organic layer was evaporated in vacuo, then, the desired product was isolated by gel permeation chromatography; yield 80\% (234 mg), yellow liquid; IR (neat): 2953, 2855, 1612, 1513, 1248, 1171, 1038, 846, 766, $691 \mathrm{~cm}^{-1} ;{ }^{1} \mathrm{H}$ NMR (400 MHz; $\left.\mathrm{CDCl}_{3}\right) \delta: 7.32-7.30$ (m, 2H), 6.93-6.89 (m, 2H), $4.42(\mathrm{~s}, 2 \mathrm{H}), 3.91(\mathrm{~s}, 2 \mathrm{H}), 3.84(\mathrm{~s}, 3 \mathrm{H}), 1.76(\mathrm{~d}, J=0.9 \mathrm{~Hz}, 3 \mathrm{H}), 1.70(\mathrm{~d}, J=0.8$ 
$\mathrm{Hz}, 3 \mathrm{H}), 1.62$ (d, $J=0.8 \mathrm{~Hz}, 2 \mathrm{H}), 0.00(\mathrm{~s}, 9 \mathrm{H}) ;{ }^{13} \mathrm{C} \mathrm{NMR}\left(100 \mathrm{MHz}, \mathrm{CDCl}_{3}\right) \delta: 160.0$ (C), 132.7 (C), $131.9(\mathrm{C}), 130.4(2 \mathrm{CH}), 123.1(\mathrm{C}), 114.6(2 \mathrm{CH}), 72.4\left(\mathrm{CH}_{2}\right), 71.6\left(\mathrm{CH}_{2}\right), 56.2\left(\mathrm{CH}_{3}\right), 26.5\left(\mathrm{CH}_{2}\right)$, $22.6\left(\mathrm{CH}_{3}\right), 17.6\left(\mathrm{CH}_{3}\right), 0.0\left(3 \mathrm{CH}_{3}\right)$; GC-MS (EI) $m / z$ (relative intensity) $292(1)[\mathrm{M}]^{+}, 121(100), 73$ (83), 209 (17); HR-MS (EI) $m / z$ calcd for $\mathrm{C}_{17} \mathrm{H}_{28} \mathrm{O}_{2} \mathrm{Si}[\mathrm{M}]^{+}:$292.1859, found 292.1858.

4g: The reaction and purification were performed using the typical reaction and isolation procedure; yield 78\% (231 mg), pale yellow liquid; IR (neat): 2954, 2857, 1491, 1248, 1163, 1087, 845, 763, $692 \mathrm{~cm}^{-1} ;{ }^{1} \mathrm{H}$ NMR $\left(400 \mathrm{MHz} ; \mathrm{CDCl}_{3}\right) \delta: 7.35-7.29(\mathrm{~m}, 4 \mathrm{H}), 4.44(\mathrm{~s}, 2 \mathrm{H}), 3.93(\mathrm{~s}, 2 \mathrm{H}), 1.75(\mathrm{~d}, J=$ $0.9 \mathrm{~Hz}, 3 \mathrm{H}), 1.70(\mathrm{~d}, J=0.8 \mathrm{~Hz}, 3 \mathrm{H}), 1.61(\mathrm{~d}, J=0.9 \mathrm{~Hz}, 2 \mathrm{H}), 0.00$ (s, 9H); ${ }^{13} \mathrm{C} \mathrm{NMR}(100 \mathrm{MHz}$; $\left.\mathrm{CDCl}_{3}\right) \delta: 138.3(\mathrm{C}), 134.1(\mathrm{C}), 133.1(\mathrm{C}), 130.0(2 \mathrm{CH}), 129.3(2 \mathrm{CH}), 122.9(\mathrm{C}), 72.0\left(\mathrm{CH}_{2}\right), 71.9$ $\left(\mathrm{CH}_{2}\right), 26.5\left(\mathrm{CH}_{2}\right), 22.6\left(\mathrm{CH}_{3}\right), 17.5\left(\mathrm{CH}_{3}\right), 0.0\left(3 \mathrm{CH}_{3}\right)$; GC-MS (EI) $\mathrm{m} / z$ (relative intensity) 296 (1) $[\mathrm{M}]^{+}, 73$ (100), 82 (60), 125 (40); HR-MS (EI) m/z calcd for $\mathrm{C}_{16} \mathrm{H}_{25} \mathrm{ClOSi}[\mathrm{M}]^{+}: 296.1363$, found 296.1362 .

4h: The reaction and purification were performed using the typical reaction and isolation procedure; yield 56\% (185 mg), colorless liquid; IR (neat): 2953, 2860, 1456, 1381, 1326, 1249, 1161, 1120, 1066, 1019, 791, 775, $692 \mathrm{~cm}^{-1} ;{ }^{1} \mathrm{H}$ NMR $\left(400 \mathrm{MHz} ; \mathrm{CDCl}_{3}\right) \delta: 7.62(\mathrm{~d}, J=8.0 \mathrm{~Hz}, 2 \mathrm{H}), 7.49$ (d, $J$ $=8.0 \mathrm{~Hz}, 2 \mathrm{H}), 4.53(\mathrm{~s}, 2 \mathrm{H}), 3.97(\mathrm{~s}, 2 \mathrm{H}), 1.77(\mathrm{~d}, J=0.9 \mathrm{~Hz}, 3 \mathrm{H}), 1.71(\mathrm{~d}, J=0.8 \mathrm{~Hz}, 3 \mathrm{H}), 1.62(\mathrm{~d}$, $J=0.7 \mathrm{~Hz}, 2 \mathrm{H}), 0.00(\mathrm{~s}, 9 \mathrm{H}) ;{ }^{13} \mathrm{C} \mathrm{NMR}\left(100 \mathrm{MHz} ; \mathrm{CDCl}_{3}\right) \delta: 144.0$ (C) 133.3 (C), $130.6\left({ }^{2} J_{\mathrm{C}-\mathrm{F}}=\right.$ $32.3 \mathrm{~Hz}), 128.7(2 \mathrm{CH}), 126.2\left({ }^{3} J_{\mathrm{C}-\mathrm{F}}=3.7 \mathrm{~Hz}\right), 125.2\left({ }^{1} J_{\mathrm{C}-\mathrm{F}}=273.2 \mathrm{~Hz}\right), 122.8(\mathrm{C}), 72.3\left(\mathrm{CH}_{2}\right), 71.9$ $\left(\mathrm{CH}_{2}\right), 26.6\left(\mathrm{CH}_{2}\right), 22.6\left(\mathrm{CH}_{3}\right), 17.5\left(\mathrm{CH}_{3}\right), 0.0\left(3 \mathrm{CH}_{3}\right)$; GC-MS (EI) $\mathrm{m} / z$ (relative intensity) 330 (1) $[\mathrm{M}]^{+}, 82$ (100), 73 (90), 140 (27); HR-MS (EI) $\mathrm{m} / z$ calcd for $\mathrm{C}_{17} \mathrm{H}_{25} \mathrm{~F}_{3} \mathrm{OSi}[\mathrm{M}]^{+}:$330.1627, found 330.1626.

4i: The reaction and purification were performed using the typical reaction and isolation procedure; yield 66\% (182 mg), yellow liquid; IR (neat): 2953, 2861, 1451, 1368, 1246, 1164, 1089, 1040, 850, 761, $695 \mathrm{~cm}^{-1} ;{ }^{1} \mathrm{H}$ NMR (400 MHz; $\left.\mathrm{CDCl}_{3}\right) \delta: 7.46-7.36(\mathrm{~m}, 5 \mathrm{H}), 4.46$ (q, $\left.J=6.5 \mathrm{~Hz}, 1 \mathrm{H}\right), 3.83-$ $3.77(\mathrm{~m}, 2 \mathrm{H}), 1.82(\mathrm{~d}, J=0.6 \mathrm{~Hz}, 3 \mathrm{H}), 1.75$ (s, 3H), 1.59 (s, 2H), 1.56 (d, J = 6.5 Hz, 3H), 0.00 (s, 9H); ${ }^{13} \mathrm{C} \mathrm{NMR}\left(100 \mathrm{MHz} ; \mathrm{CDCl}_{3}\right) \delta: 145.3(\mathrm{C}), 132.8(\mathrm{C}), 129.3(2 \mathrm{CH}), 128.3(\mathrm{CH}), 127.3(2 \mathrm{CH})$, $123.3(\mathrm{C}), 78.1(\mathrm{CH}), 70.6\left(\mathrm{CH}_{2}\right), 26.5\left(\mathrm{CH}_{2}\right), 25.2\left(\mathrm{CH}_{3}\right), 22.6\left(\mathrm{CH}_{3}\right), 17.8\left(\mathrm{CH}_{3}\right), 0.0\left(3 \mathrm{CH}_{3}\right)$; GCMS (EI) $m / z$ (relative intensity) 276 (1) [M] $]^{+}, 73$ (100), 105 (50), 82 (25); HR-MS (EI) $m / z$ calcd for $\mathrm{C}_{17} \mathrm{H}_{28} \mathrm{OSi}[\mathrm{M}]^{+}:$276.1909, found 276.1904. 
$\mathbf{4 j}$ : The reaction and purification were performed using the typical reaction and isolation procedure; yield 62\% (179 mg), colorless liquid; IR (neat): 2953, 2856, 1653, 1448, 1352, 1248, 1164, 1072, 965, 842, 742, $691 \mathrm{~cm}^{-1} ;{ }^{1} \mathrm{H}$ NMR $\left(400 \mathrm{MHz} ; \mathrm{CDCl}_{3}\right) \delta: 7.41-7.22(\mathrm{~m}, 5 \mathrm{H}), 6.61(\mathrm{~d}, J=15.9 \mathrm{~Hz}$, $1 \mathrm{H}), 6.32(\mathrm{dt}, J=15.9,6.0 \mathrm{~Hz}, 1 \mathrm{H}), 4.09$ (dd, $J=6.1,1.4 \mathrm{~Hz}, 2 \mathrm{H}), 3.94(\mathrm{~s}, 2 \mathrm{H}), 1.75$ (s, 3H), 1.69 (s, 3H), $1.66(\mathrm{~s}, 2 \mathrm{H}), 0.00(\mathrm{~s}, 9 \mathrm{H}) ;{ }^{13} \mathrm{C} \mathrm{NMR}\left(100 \mathrm{MHz} ; \mathrm{CDCl}_{3}\right) \delta: 137.8(\mathrm{C}), 133.0(\mathrm{CH}), 132.7(\mathrm{C})$, $129.4(2 \mathrm{CH}), 128.4(\mathrm{CH}), 127.6(\mathrm{CH}), 127.3(2 \mathrm{CH}), 123.1(\mathrm{C}), 71.9\left(\mathrm{CH}_{2}\right), 71.2\left(\mathrm{CH}_{2}\right), 26.5\left(\mathrm{CH}_{2}\right)$, $22.6\left(\mathrm{CH}_{3}\right), 17.5\left(\mathrm{CH}_{3}\right), 0.0\left(3 \mathrm{CH}_{3}\right)$; GC-MS (EI) $m / z$ (relative intensity) 288 (1) $[\mathrm{M}]^{+}, 73$ (100), 117 (51), 115 (16); HR-MS (EI) $m / z$ calcd for $\mathrm{C}_{18} \mathrm{H}_{28} \mathrm{OSi}[\mathrm{M}]^{+}: 288.1909$, found 288.1907 .

4k: The reaction and purification were performed using the typical reaction and isolation procedure; yield 65\% (203 mg), colorless liquid; IR (neat): 3043, 2951, 2857, 1456, 1247, 1163, 1065, 844, 807, 764, $694 \mathrm{~cm}^{-1} ;{ }^{1} \mathrm{H}$ NMR (400MHz; $\left.\mathrm{CDCl}_{3}\right) \delta: 8.22(\mathrm{~d}, J=8.0 \mathrm{~Hz}, 1 \mathrm{H}), 7.92-7.85(\mathrm{~m}, 2 \mathrm{H}), 7.60-7.46$ $(\mathrm{m}, 4 \mathrm{H}), 4.97(\mathrm{~s}, 2 \mathrm{H}), 4.04(\mathrm{~s}, 2 \mathrm{H}), 1.80(\mathrm{~s}, 8 \mathrm{H}), 1.73(\mathrm{~s}, 8 \mathrm{H}), 1.65(\mathrm{~s}, 8 \mathrm{H}), 0.00(\mathrm{~s}, 9 \mathrm{H}) ;{ }^{13} \mathrm{C} \mathrm{NMR}$ $\left(100 \mathrm{MHz} ; \mathrm{CDCl}_{3}\right) \delta: 135.2(\mathrm{C}), 134.7(\mathrm{C}), 132.9(\mathrm{C}), 132.8(\mathrm{C}), 129.4(\mathrm{CH}), 129.3(\mathrm{CH}), 127.5(\mathrm{CH})$, 127.0 (CH), $126.6(\mathrm{CH}), 126.1(\mathrm{CH}), 125.2(\mathrm{CH}), 123.1(\mathrm{CH}), 72.1\left(\mathrm{CH}_{2}\right), 71.3\left(\mathrm{CH}_{2}\right), 26.5\left(\mathrm{CH}_{2}\right)$, $22.6\left(\mathrm{CH}_{3}\right), 17.7\left(\mathrm{CH}_{3}\right), 0.0\left(3 \mathrm{CH}_{3}\right)$; GC-MS (EI) $m / z$ (relative intensity) 312 (1) $[\mathrm{M}]^{+}, 73$ (100), 141 (94), 230 (37); HR-MS (EI) $\mathrm{m} / z$ calcd for $\mathrm{C}_{20} \mathrm{H}_{28} \mathrm{OSi}[\mathrm{M}]^{+}:$312.1909, found 312.1913.

4l: The reaction and purification were performed using the typical reaction and isolation procedure; yield 68\% (208 mg), yellow liquid; IR (neat): 2954, 2857, 1490, 1443, 1245, 1164, 1060, 931, 845, 807, 764, $694 \mathrm{~cm}^{-1}$; ${ }^{1} \mathrm{H}$ NMR (400 MHz; $\left.\mathrm{CDCl}_{3}\right) \delta:$ 6.90-6.89 (m, 1H), 6.84-6.78 (m, 2H), 5.97 (s, 2H), $4.37(\mathrm{~s}, 2 \mathrm{H}), 3.90(\mathrm{~s}, 2 \mathrm{H}), 1.75(\mathrm{~d}, J=0.9 \mathrm{~Hz}, 3 \mathrm{H}), 1.70(\mathrm{~d}, J=0.8 \mathrm{~Hz}, 3 \mathrm{H}), 1.62$ (d, $J=0.8 \mathrm{~Hz}$, 2H), 0.00 (s, 9H); ${ }^{13} \mathrm{C}$ NMR (100 MHz; $\left.\mathrm{CDCl}_{3}\right) \delta: 148.6$ (C), 147.8 (C), 133.7 (C), 132.8 (C), 123.0 (C), $122.3(\mathrm{CH}), 109.5(\mathrm{CH}), 108.9(\mathrm{CH}), 101.8\left(\mathrm{CH}_{2}\right), 72.6\left(\mathrm{CH}_{2}\right), 71.7\left(\mathrm{CH}_{2}\right), 26.5\left(\mathrm{CH}_{2}\right), 22.6$ $\left(\mathrm{CH}_{3}\right), 17.6\left(\mathrm{CH}_{3}\right), 0.0\left(3 \mathrm{CH}_{3}\right)$; GC-MS (EI) m/z (relative intensity) 306 (1) [M] $]^{+}, 73$ (100), 135 (26), 224 (18); HR-MS (EI) $m / z$ calcd for $\mathrm{C}_{17} \mathrm{H}_{26} \mathrm{O}_{3} \mathrm{Si}[\mathrm{M}]^{+}: 306.1651$, found 306.1646.

4m: The reaction and purification were performed using the typical reaction and isolation procedure; yield 52\% (131 mg), yellow liquid; IR (neat); 2951, 2857, 1436, 1248, 1150, 1060, 844, 809, 733, $691 \mathrm{~cm}^{-1} ;{ }^{1} \mathrm{H}$ NMR $\left(400 \mathrm{MHz} ; \mathrm{CDCl}_{3}\right) \delta: 7.43-7.42(\mathrm{~m}, 1 \mathrm{H}), 6.37-6.32(\mathrm{~m}, 2 \mathrm{H}), 4.41$ (s, 2H), 3.93 (s, 2H), $1.73(\mathrm{~d}, J=0.9 \mathrm{~Hz}, 3 \mathrm{H}), 1.69$ (d, $J=0.8 \mathrm{~Hz}, 3 \mathrm{H}), 1.62(\mathrm{~d}, J=0.8 \mathrm{~Hz}, 2 \mathrm{H}), 0.00(\mathrm{~s}, 9 \mathrm{H}) ;{ }^{13} \mathrm{C}$

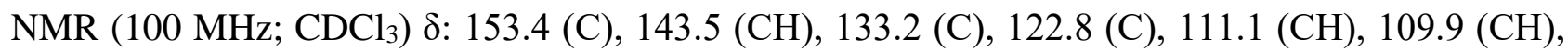
$71.7\left(\mathrm{CH}_{2}\right), 64.4\left(\mathrm{CH}_{2}\right), 26.5\left(\mathrm{CH}_{2}\right), 22.6\left(\mathrm{CH}_{3}\right), 17.5\left(\mathrm{CH}_{3}\right), 0.0\left(3 \mathrm{CH}_{3}\right) ; \mathrm{GC}-\mathrm{MS}(\mathrm{EI}) \mathrm{m} / z$ (relative 
intensity) 252 (1) $[\mathrm{M}]^{+}, 73$ (100), 81 (68), 155 (48); HR-MS (EI) $\mathrm{m} / \mathrm{z}$ calcd for $\mathrm{C}_{14} \mathrm{H}_{24} \mathrm{O}_{2} \mathrm{Si}[\mathrm{M}]^{+}$: 252.1546, found 252.1543.

4n: The reaction and purification were performed using the typical reaction and isolation procedure; yield 52\% (140 mg), pale yellow liquid; IR (neat): 2952, 2857, 1248, 1163, 1059, 847, 764, $697 \mathrm{~cm}^{-}$ 1; ${ }^{1} \mathrm{H}$ NMR (400 MHz; $\mathrm{CDCl}_{3}$ ) $\delta: 7.96$ (d, $\left.J=7.7 \mathrm{~Hz}, 2 \mathrm{H}\right), 7.31-7.24(\mathrm{~m}, 4 \mathrm{H}), 7.10-7.06$ (m, 2H), 4.69 (s, 2H), 1.85 (s, 2H), 1.59 (s, 3H), 1.18 (s, 3H), 0.00 (s, 9H); $\left.{ }^{13} \mathrm{C} \mathrm{NMR} \mathrm{(100} \mathrm{MHz;} \mathrm{CDCl}_{3}\right) \delta$ : $142.6(\mathrm{C}), 133.2(\mathrm{C}), 127.4(\mathrm{CH}), 127.2(\mathrm{CH}), 126.5(\mathrm{CH}), 122.8(\mathrm{C}), 71.6\left(\mathrm{CH}_{2}\right), 67.0\left(\mathrm{CH}_{2}\right), 26.5$ $\left(\mathrm{CH}_{2}\right), 22.6\left(\mathrm{CH}_{3}\right), 17.5\left(\mathrm{CH}_{3}\right), 0.0\left(3 \mathrm{CH}_{3}\right)$; GC-MS (EI) m/z (relative intensity) 268 (1) [M] $]^{+}, 73$ (100), 97 (67), 186 (27); HR-MS (EI) m/z calcd for $\mathrm{C}_{14} \mathrm{H}_{24} \mathrm{OSSi}[\mathrm{M}]^{+}:$268.1317, found 268.1311.

4o: The reaction was performed using the typical reaction procedure. The resulting mixture was filtered and evaporated in vacuo. The solution was then purified by silica gel column chromatography (with hexane as the eluent) and gel permeation chromatography; yield 56\% (144 mg), colorless liquid; IR (neat): 2956, 2858, 1456, 1163, 1094, 843, 764, $694 \mathrm{~cm}^{-1} ;{ }^{1} \mathrm{H} \mathrm{NMR}\left(400 \mathrm{MHz} ; \mathrm{CDCl}_{3}\right) \delta: 3.85$ (s, 2H), 3.35 (t, $J=6.6 \mathrm{~Hz}, 2 \mathrm{H}), 1.70(\mathrm{~s}, 3 \mathrm{H}), 1.66(\mathrm{~s}, 3 \mathrm{H}), 1.63(\mathrm{~s}, 2 \mathrm{H}), 1.57$ (quin, 2H), 1.37-1.27 (m, $6 \mathrm{H}), 0.89(\mathrm{t}, J=6.9 \mathrm{~Hz}, 3 \mathrm{H}), 0.00(\mathrm{~s}, 9 \mathrm{H}) ;{ }^{13} \mathrm{C} \mathrm{NMR}\left(100 \mathrm{MHz} ; \mathrm{CDCl}_{3}\right) \delta: 72.4\left(\mathrm{CH}_{2}\right), 70.9\left(\mathrm{CH}_{2}\right)$, 32.6 $\left(\mathrm{CH}_{2}\right), 30.7\left(\mathrm{CH}_{2}\right), 26.9\left(\mathrm{CH}_{2}\right), 26.4\left(\mathrm{CH}_{2}\right), 23.5\left(\mathrm{CH}_{2}\right), 22.5\left(\mathrm{CH}_{3}\right), 17.3\left(\mathrm{CH}_{3}\right), 14.9\left(\mathrm{CH}_{3}\right), 0.0$ $\left(3 \mathrm{CH}_{3}\right)$; GC-MS (EI) m/z (relative intensity) 256 (1) $[\mathrm{M}]^{+}, 82$ (100), 73 (74), 67 (31); HR-MS (EI) $m / z$ calcd for $\mathrm{C}_{15} \mathrm{H}_{32} \mathrm{OSi}[\mathrm{M}]^{+}:$256.2222, found 256.2219.

4p: The reaction was performed using the typical reaction procedure. The resulting mixture was filtered and evaporated in vacuo. The solution was then purified by silica gel column chromatography (with hexane as the eluent) and vacuum distilled $(0.5 \mathrm{mmHg})$ with a Kugelrohr apparatus at $150{ }^{\circ} \mathrm{C}$ for 45 min; yield 80\% (326 mg), colorless liquid; IR (neat): 2955, 2851, 1464, 1248, 1164, 1092, 847, 762, $691 \mathrm{~cm}^{-1} ;{ }^{1} \mathrm{H}$ NMR (400 MHz; $\left.\mathrm{CDCl}_{3}\right) \delta: 3.85(\mathrm{~s}, 2 \mathrm{H}), 3.35(\mathrm{t}, J=6.7 \mathrm{~Hz}, 2 \mathrm{H}), 1.70(\mathrm{~d}, J=0.8$ $\mathrm{Hz}, 3 \mathrm{H}), 1.66(\mathrm{~d}, J=0.7 \mathrm{~Hz}, 3 \mathrm{H}), 1.63(\mathrm{~d}, J=0.7 \mathrm{~Hz}, 2 \mathrm{H}), 1.59-1.55(\mathrm{~m}, 2 \mathrm{H}), 1.33-1.26(\mathrm{~m}, 30 \mathrm{H})$, $0.88(\mathrm{t}, J=6.9 \mathrm{~Hz}, 3 \mathrm{H}), 0.00(\mathrm{~s}, 9 \mathrm{H}) ;{ }^{13} \mathrm{C} \mathrm{NMR}\left(100 \mathrm{MHz}, \mathrm{CDCl}_{3}\right) \delta: 131.9$ (C), $123.4(\mathrm{C}), 72.4$ $\left(\mathrm{CH}_{2}\right), 70.9\left(\mathrm{CH}_{2}\right), 32.8\left(\mathrm{CH}_{2}\right), 30.8\left(\mathrm{CH}_{2}\right), 30.6\left(7 \mathrm{CH}_{2}\right), 30.5\left(\mathrm{CH}_{2}\right), 30.5\left(\mathrm{CH}_{2}\right), 30.5\left(\mathrm{CH}_{2}\right), 30.4$ $\left(\mathrm{CH}_{2}\right), 30.2\left(\mathrm{CH}_{2}\right), 27.2\left(\mathrm{CH}_{2}\right), 26.4\left(\mathrm{CH}_{2}\right), 23.6\left(\mathrm{CH}_{2}\right), 22.5\left(\mathrm{CH}_{3}\right), 17.3\left(\mathrm{CH}_{3}\right), 15.0\left(\mathrm{CH}_{3}\right), 0.0$ $\left(3 \mathrm{CH}_{3}\right)$; GC-MS (EI) m/z (relative intensity) 424 (1) $[\mathrm{M}]^{+}, 82$ (100), 73 (41), 83 (11); HR-MS (EI) $m / z$ calcd for $\mathrm{C}_{27} \mathrm{H}_{56} \mathrm{OSi}[\mathrm{M}]^{+}:$424.4100, found 424.4091 . 
4q: The reaction was performed using the typical reaction procedure. The resulting mixture was filtered and evaporated in vacuo. The solution was then purified by silica gel column chromatography (with hexane as the eluent) and vacuum distilled using a Kugelrohr apparatus at $0.5 \mathrm{mmHg}\left(100{ }^{\circ} \mathrm{C}\right.$, $30 \mathrm{~min}$, then $90{ }^{\circ} \mathrm{C}, 40 \mathrm{~min}$ ); 51\% (115 mg), colorless liquid; IR (neat): 2954, 2856, 1248, 1164 , 1083, 845, 762, $694 \mathrm{~cm}^{-1} ;{ }^{1} \mathrm{H}$ NMR (400 MHz; $\left.\mathrm{CDCl}_{3}\right) \delta: 3.89$ (s, 2H), 3.19 (d, J=6.9 Hz, 2H), 1.71 $(\mathrm{d}, J=0.9 \mathrm{~Hz}, 3 \mathrm{H}), 1.66(\mathrm{~d}, J=0.8 \mathrm{~Hz}, 3 \mathrm{H}), 1.63(\mathrm{~d}, J=0.7 \mathrm{~Hz}, 2 \mathrm{H}), 1.12-1.02(\mathrm{~m}, 1 \mathrm{H}), 0.53-0.51$ (m, 2H), 0.20-0.18 (m, 2H), $\left.0.00(\mathrm{~s}, 9 \mathrm{H}) ;{ }^{13} \mathrm{C} \mathrm{NMR} \mathrm{(100} \mathrm{MHz;} \mathrm{CDCl}_{3}\right) \delta: 132.2(\mathrm{C}), 123.3(\mathrm{C}), 75.4$ $\left(\mathrm{CH}_{2}\right), 72.2\left(\mathrm{CH}_{2}\right), 26.4\left(\mathrm{CH}_{2}\right), 22.5\left(\mathrm{CH}_{3}\right), 17.3\left(\mathrm{CH}_{3}\right), 11.6(\mathrm{CH}), 3.9\left(\mathrm{CH}_{2}\right), 0.0\left(3 \mathrm{CH}_{3}\right) ; \mathrm{GC}-\mathrm{MS}$ (EI) $\mathrm{m} / \mathrm{z}$ (relative intensity) 226 (1) $[\mathrm{M}]^{+}, 73$ (100), 82 (92), 67 (37); HR-MS (EI) $\mathrm{m} / z$ calcd for $\mathrm{C}_{13} \mathrm{H}_{26} \mathrm{OSi}[\mathrm{M}]^{+}:$226.1753, found 226.1750.

4r: The reaction was performed using the typical reaction procedure. The resulting mixture was filtered and evaporated in vacuo. The solution was then purified by silica gel column chromatography (with hexane as the eluent) and gel permeation chromatography; yield 53\% (287 mg), colorless oil like grease; IR (neat): 2948, 2900, 2862, 1729, 1658, 1247, 1165, 1085, 846, 734, 692; ${ }^{1} \mathrm{H}$ NMR (400

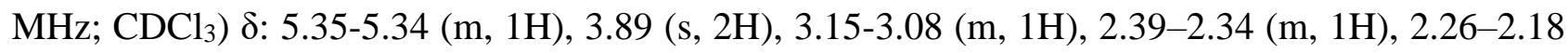
(m, 1H), 2.03-1.80 (m, 5H), $1.71(\mathrm{~d}, J=0.7 \mathrm{~Hz}, 3 \mathrm{H}), 1.65-0.86(\mathrm{~m}, 38 \mathrm{H}), 0.68(\mathrm{~s}, 3 \mathrm{H}), 0.00(\mathrm{~s}, 9 \mathrm{H})$; ${ }^{13} \mathrm{C}$ NMR (100 MHz; $\left.\mathrm{CDCl}_{3}\right) \delta: 142.1(\mathrm{C}), 131.9(\mathrm{C}), 123.5(\mathrm{C}), 122.1(\mathrm{CH}), 79.0(\mathrm{CH}), 69.7\left(\mathrm{CH}_{2}\right)$, 57.6 (CH), $57.0(\mathrm{CH}), 51.0(\mathrm{CH}), 43.1(\mathrm{C}), 40.6\left(\mathrm{CH}_{2}\right), 40.3\left(\mathrm{CH}_{2}\right), 40.1\left(\mathrm{CH}_{2}\right), 38.2\left(\mathrm{CH}_{2}\right), 37.7(\mathrm{C})$, 37.0 $\left(\mathrm{CH}_{2}\right), 36.6(\mathrm{CH}), 32.8\left(\mathrm{CH}_{2}\right), 32.7(\mathrm{CH}), 29.3\left(\mathrm{CH}_{2}\right), 29.1\left(\mathrm{CH}_{2}\right), 28.8\left(\mathrm{CH}_{3}\right), 26.4\left(\mathrm{CH}_{2}\right), 25.1$ $\left(\mathrm{CH}_{2}\right), 24.6\left(\mathrm{CH}_{2}\right), 23.7\left(\mathrm{CH}_{3}\right), 23.4\left(\mathrm{CH}_{2}\right), 22.5\left(\mathrm{CH}_{3}\right), 21.9\left(\mathrm{CH}_{2}\right), 20.2\left(\mathrm{CH}_{3}\right), 19.5\left(\mathrm{CH}_{3}\right), 17.5$ $\left(\mathrm{CH}_{3}\right), 12.7\left(\mathrm{CH}_{3}\right), 0.0\left(3 \mathrm{CH}_{3}\right)$; GC-MS (EI) $\mathrm{m} / z$ (relative intensity) 540 (1) [M] $]^{+}, 73$ (100), 155 (19), 139 (15); HR-MS (EI) $m / z$ calcd for $\mathrm{C}_{36} \mathrm{H}_{64} \mathrm{OSi}[\mathrm{M}]^{+}:$540.4726, found 540.4729 .

4s: The reaction was performed using the typical reaction procedure. The resulting mixture was filtered and evaporated in vacuo. The solution was then purified by silica gel column chromatography (with hexane as the eluent) and gel permeation chromatography; yield 64\% (199 mg), pale yellow liquid; IR (neat): 2952, 2924, 2867, 1455, 1383, 1248, 1166, 1054, 845, $689 \mathrm{~cm}^{-1}$; ${ }^{1} \mathrm{H}$ NMR (400 $\left.\mathrm{MHz} ; \mathrm{CDCl}_{3}\right) \delta: 4.04(\mathrm{~d}, J=9.9 \mathrm{~Hz}, 1 \mathrm{H}), 3.68(\mathrm{~d}, J=10.1 \mathrm{~Hz}, 1 \mathrm{H}), 2.99(\mathrm{td}, J=10.6,4.1 \mathrm{~Hz}, 1 \mathrm{H})$, 2.34-2.26 (m, 1H), 2.12-2.09 (m, 1H), 2.01-1.58 (m, 11H), 1.36-1.33 (m, 1H), 1.28-1.20 (m, 1H), 1.01-0.76 (m, 12H), $0.00(\mathrm{~s}, 9 \mathrm{H}) ;{ }^{13} \mathrm{C} \mathrm{NMR}\left(100 \mathrm{MHz} ; \mathrm{CDCl}_{3}\right) \delta: 132.0(\mathrm{C}), 123.6(\mathrm{C}), 79.2(\mathrm{CH})$, $70.3\left(\mathrm{CH}_{2}\right), 49.1(\mathrm{CH}), 41.3\left(\mathrm{CH}_{2}\right), 35.5\left(\mathrm{CH}_{2}\right), 32.5(\mathrm{CH}), 26.4\left(\mathrm{CH}_{2}\right), 26.1(\mathrm{CH}), 23.9\left(\mathrm{CH}_{2}\right), 23.3$ $\left(\mathrm{CH}_{3}\right), 22.4\left(\mathrm{CH}_{3}\right), 21.9\left(\mathrm{CH}_{3}\right), 17.9\left(\mathrm{CH}_{3}\right), 16.7\left(\mathrm{CH}_{3}\right), 0.0\left(3 \mathrm{CH}_{3}\right) ; \mathrm{GC}-\mathrm{MS}(\mathrm{EI}) \mathrm{m} / \mathrm{z}$ (relative 
intensity) 310 (3) $[\mathrm{M}]^{+}, 73$ (100), 82 (48), 83 (29); HR-MS (EI) $m / z$ calcd for $\mathrm{C}_{19} \mathrm{H}_{38} \mathrm{OSi}[\mathrm{M}]^{+}$: 310.2692, found 310.2691.

4t: The reaction was performed using the typical reaction procedure. The resulting mixture was filtered and evaporated in vacuo. The solution was then purified by silica gel column chromatography (with hexane as the eluent) and gel permeation chromatography; yield 33\% (102mg), colorless liquid; IR (neat): 2946, 2871, 1721, 1455, 1366, 1248, 1091, 840, $733 \mathrm{~cm}^{-1} ;{ }^{1} \mathrm{H}$ NMR (400 MHz; $\left.\mathrm{CDCl}_{3}\right) \delta$ : $3.96(\mathrm{~d}, J=10.4 \mathrm{~Hz}, 1 \mathrm{H}), 3.60(\mathrm{~d}, J=10.4 \mathrm{~Hz}, 1 \mathrm{H}), 3.58-3.58(\mathrm{~m}, 1 \mathrm{H}), 2.05-2.01(\mathrm{~m}, 1 \mathrm{H}), 1.68-1.55$ $(\mathrm{m}, 12 \mathrm{H}), 1.39-1.32(\mathrm{~m}, 1 \mathrm{H}), 0.90-0.78(\mathrm{~m}, 12 \mathrm{H}), 0.00(\mathrm{~s}, 9 \mathrm{H}) ;{ }^{13} \mathrm{C} \mathrm{NMR}\left(100 \mathrm{MHz} ; \mathrm{CDCl}_{3}\right) \delta: 130.8$ (C), $124.2(\mathrm{C}), 75.2(\mathrm{CH}), 70.0\left(\mathrm{CH}_{2}\right), 49.4(\mathrm{CH}), 38.6\left(\mathrm{CH}_{2}\right), 36.1\left(\mathrm{CH}_{2}\right), 29.5\left(\mathrm{CH}_{3}\right), 26.9(\mathrm{CH})$, $26.3\left(\mathrm{CH}_{2}\right), 25.7\left(\mathrm{CH}_{2}\right), 23.4\left(\mathrm{CH}_{3}\right), 22.4(\mathrm{CH}), 22.0\left(\mathrm{CH}_{3}\right), 21.9\left(\mathrm{CH}_{3}\right), 17.5\left(\mathrm{CH}_{3}\right), 0.0\left(3 \mathrm{CH}_{3}\right)$; GCMS (EI) $m / z$ (relative intensity) 310 (1) [M] $]^{+}, 73$ (100), 82 (40), 157 (22); HR-MS (EI) $m / z$ calcd for $\mathrm{C}_{19} \mathrm{H}_{38} \mathrm{OSi}[\mathrm{M}]^{+}:$310.2692, found 310.2684.

4u: The reaction and purification were performed using the typical reaction and isolation procedure; yield 61\% (211 mg), colorless liquid; IR (neat): 2955, 2859, 1456, 1247, 1159, 1068, 849, 732, 696 $\mathrm{cm}^{-1} ;{ }^{1} \mathrm{H}$ NMR $\left(400 \mathrm{MHz} ; \mathrm{CDCl}_{3}\right) \delta: 7.40-7.33(\mathrm{~m}, 5 \mathrm{H}), 4.49(\mathrm{~s}, 2 \mathrm{H}), 3.95(\mathrm{~s}, 2 \mathrm{H}), 2.18(\mathrm{t}, \mathrm{J}=7.5$ $\mathrm{Hz}, 2 \mathrm{H}), 2.01(\mathrm{t}, \mathrm{J}=7.7 \mathrm{~Hz}, 2 \mathrm{H}), 1.62(\mathrm{~s}, 2 \mathrm{H}), 1.47-1.34(\mathrm{~m}, 8 \mathrm{H}), 0.97(\mathrm{~m}, 6 \mathrm{H}), 0.00(\mathrm{~s}, 9 \mathrm{H}) ;{ }^{13} \mathrm{C}$

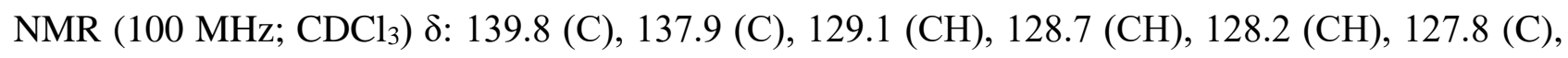
$72.2\left(\mathrm{CH}_{2}\right), 69.4\left(\mathrm{CH}_{2}\right), 34.9\left(\mathrm{CH}_{2}\right), 32.3\left(\mathrm{CH}_{2}\right), 31.9\left(\mathrm{CH}_{2}\right), 30.1\left(\mathrm{CH}_{2}\right), 23.9\left(\mathrm{CH}_{2}\right), 23.8\left(\mathrm{CH}_{2}\right)$, $23.4\left(\mathrm{CH}_{2}\right), 14.9\left(\mathrm{CH}_{3}\right), 14.9\left(\mathrm{CH}_{3}\right), 0.0\left(3 \mathrm{CH}_{3}\right)$; GC-MS (EI) $\mathrm{m} / z$ (relative intensity) $346(1)[\mathrm{M}]^{+}$, 73 (100), 91 (65), 82 (44); HR-MS (EI) $\mathrm{m} / z$ calcd for $\mathrm{C}_{22} \mathrm{H}_{38} \mathrm{OSi}[\mathrm{M}]^{+}:$346.2692, found 346.2695. 


\section{Mechanistic Studies}

\section{4-1. Preparation of benzyl alcohol- $d_{1}$}

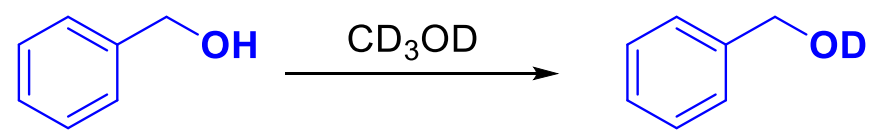

In a pear-shaped flask, benzyl alcohol (1.08 g, $10 \mathrm{mmol})$ was added to $\mathrm{CD}_{3} \mathrm{OD}(>99 \%-\mathrm{D}, 7.5 \mathrm{~mL})$, and the mixture was stirred at ambient temperature for $16 \mathrm{~h}$. Then, the reaction mixture was filtered and the solution was evaporated in vacuo. The desired product $\left(\mathbf{2 a}-d_{1}\right)$ was obtained as a yellow oil (89\% D-incorporation at the $\mathrm{OH}$ moiety determined by ${ }^{1} \mathrm{H}$ NMR $400 \mathrm{MHz}$ in $\mathrm{CDCl}_{3}$ ).

\section{4-2. Deuterium Labeling Experiments $\left(2 \mathrm{a}-\boldsymbol{d}_{1}\right)$}

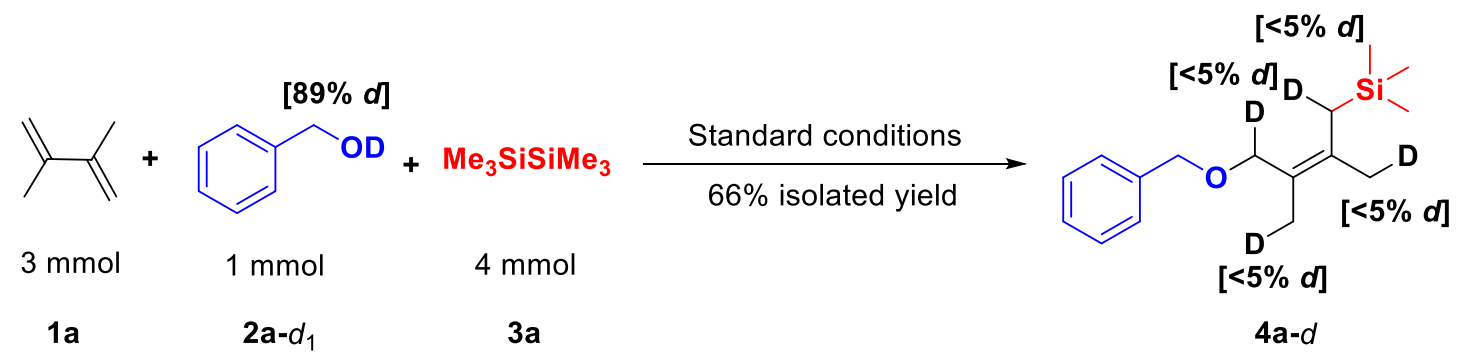

$\operatorname{Pd}(\mathrm{dba})_{2}(29 \mathrm{mg}, 5 \mathrm{~mol} \%), \mathrm{CuBr}_{2}(22 \mathrm{mg}, 10 \mathrm{~mol} \%)$, and 1,4-benzoquinone (43 mg, $40 \mathrm{~mol} \%$ ) were stirred in DMF (3 mL) for $30 \mathrm{~min}$ at room temperature. Then, 2,3-dimethyl-1,3-butadiene (1a; 246 $\mathrm{mg}, 3 \mathrm{mmol})$, benzyl alcohol- $d_{1}\left(\mathbf{2 a}-d_{1} ; 109 \mathrm{mg}, 1 \mathrm{mmol}\right)$, and hexamethyldisilane (3a; $585 \mathrm{mg}, 4$ mmol) were added to the solution. The reaction mixture was stirred for $16 \mathrm{~h}$ at $70{ }^{\circ} \mathrm{C}$ (oil bath temperature) under $\mathrm{O}_{2}$ (balloon). Then, the reaction was quenched with toluene $(10 \mathrm{~mL})$. The product, 4a- $d$, was isolated by silica gel column chromatography (with hexane as the eluent) in $66 \%$ yield (174 $\mathrm{mg}$ ) as a colorless liquid. 


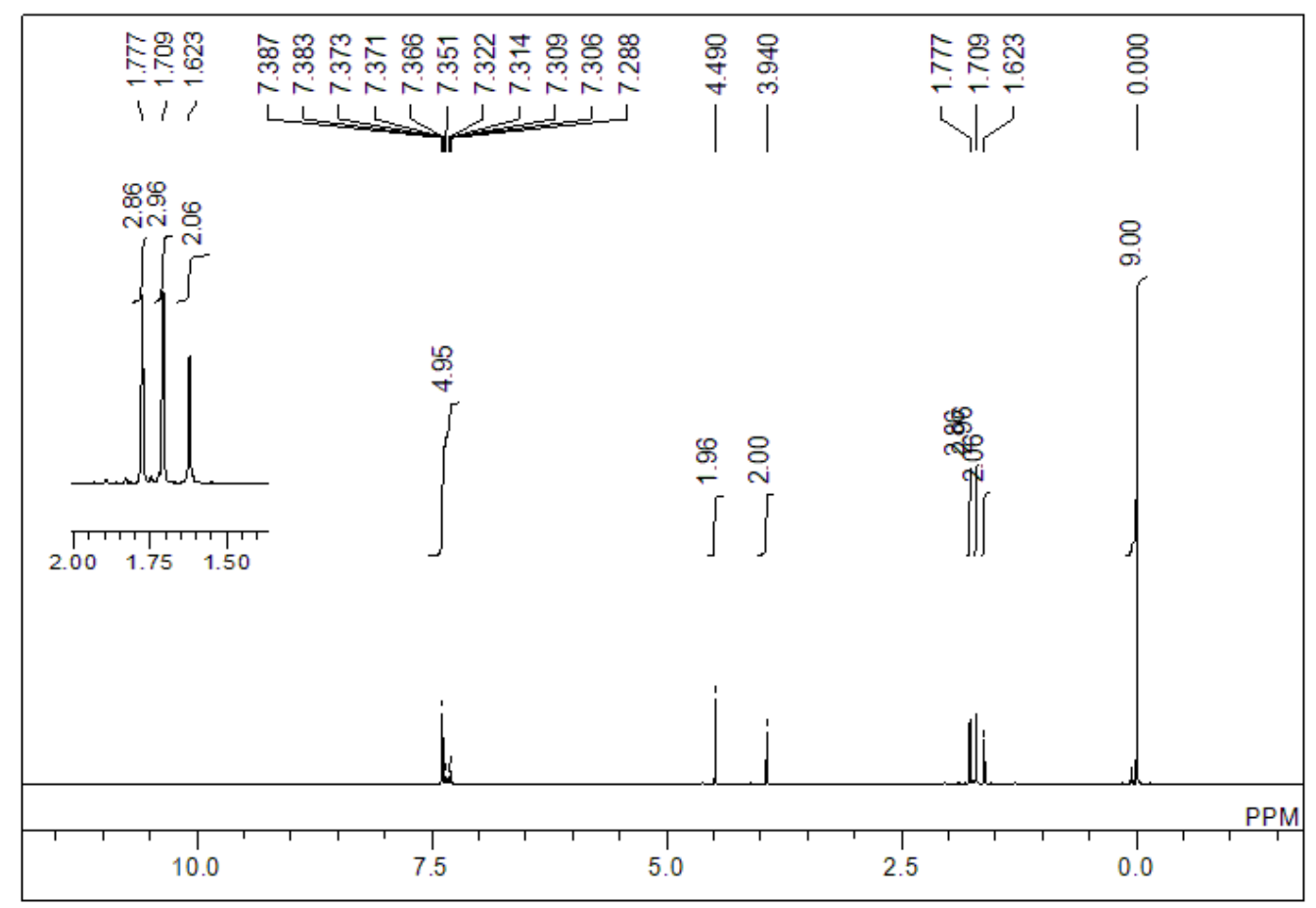

Figure S1. ${ }^{1} \mathrm{H}$ NMR (400 MHz) Spectrum of $\mathbf{4 a}-d$ in $\mathrm{CDCl}_{3}$

\section{4-3. Deuterium Labeling Experiments $\left(2 a-d_{7}\right)$}<smiles>C=C(C)C(=C)C</smiles>

$3 \mathrm{mmol}$

1a

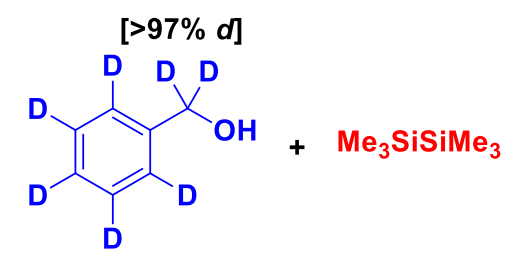

$1 \mathrm{mmol}$

$2 \mathrm{a}-d_{7}$<smiles>CC(C)[GeH3]</smiles>

$3 a$<smiles>[2H]c1c([2H])c([2H])c(C([2H])([2H])OC/C(C)=C(/C)C[Si](C)(C)C)c([2H])c1[2H]</smiles>

$4 \mathrm{a}-d_{7}$

$\operatorname{Pd}(\mathrm{dba})_{2}(29 \mathrm{mg}, 5 \mathrm{~mol} \%), \mathrm{CuBr}_{2}(22 \mathrm{mg}, 10 \mathrm{~mol} \%)$, and 1,4-benzoquinone (43 mg, $40 \mathrm{~mol} \%$ ) were stirred in DMF (3 mL) for $30 \mathrm{~min}$ at room temperature. Then, 2,3-dimethyl-1,3-butadiene (1a; 246 $\mathrm{mg}, 3 \mathrm{mmol})$, benzyl alcohol- $d_{7}\left(\mathbf{2 a}-d_{7} ; 115 \mathrm{mg}, 1 \mathrm{mmol}\right)$, and hexamethyldisilane (3a; $585 \mathrm{mg}, 4$ mmol) were added to the solution. The reaction mixture was stirred for $16 \mathrm{~h}$ at $70{ }^{\circ} \mathrm{C}$ (oil bath temperature) under $\mathrm{O}_{2}$ (balloon). Then, the reaction was quenched with toluene (10 mL). The product, 4a- $d_{7}$, was isolated by silica gel column chromatography (with hexane as the eluent) in $65 \%$ yield $(175 \mathrm{mg})$ as a colorless liquid. 


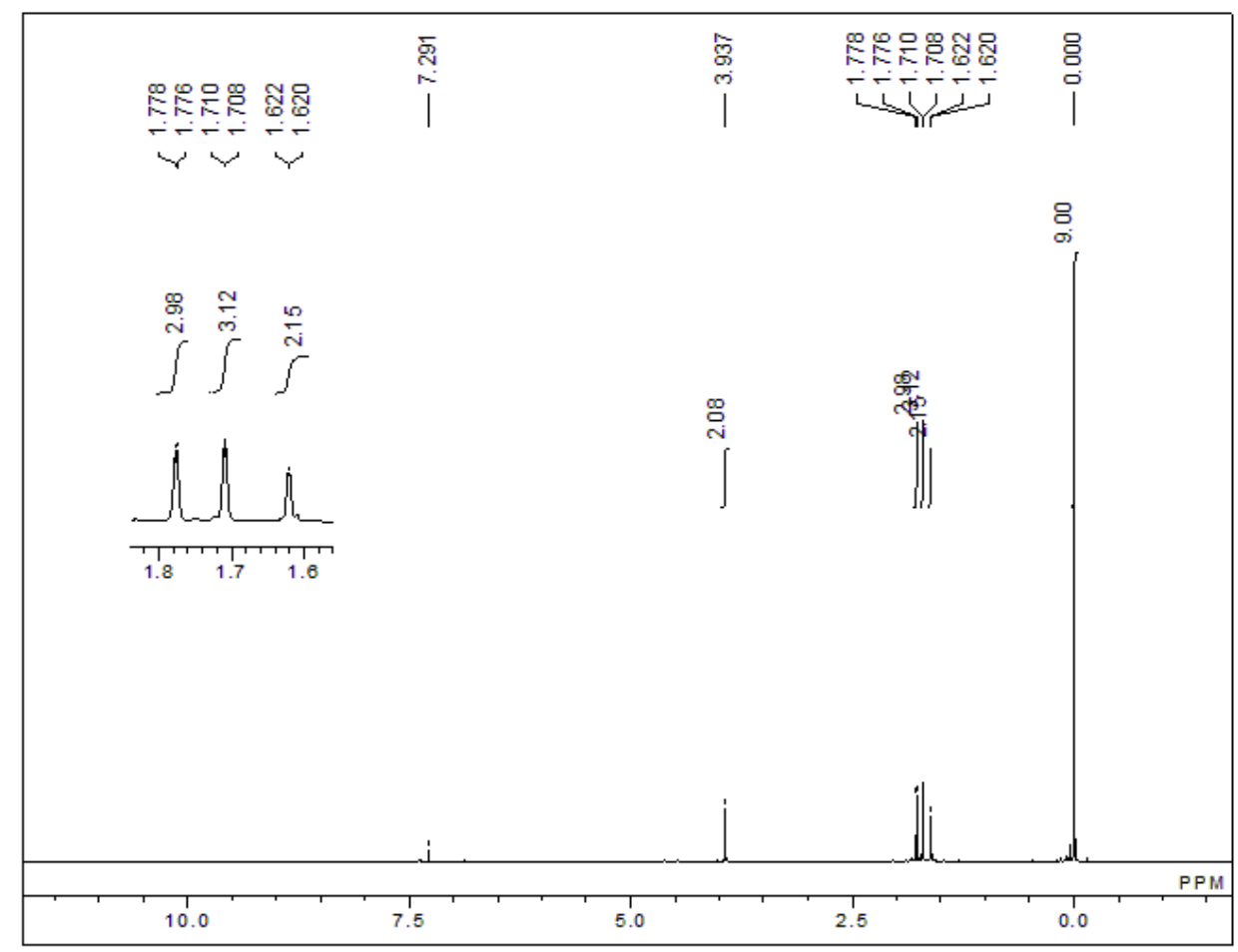

Figure S2. ${ }^{1} \mathrm{H}$ NMR (400 MHz) Spectrum of $\mathbf{4 a}-d_{7}$ in $\mathrm{CDCl}_{3}$

\section{4-4 ${ }^{29}$ Si NMR (DEPT) Measurement of Reaction Mixture}

The silylalkoxylation with 1a, 2a, and 3a was performed under optimized condition (Table 1, entry 1). After 2 hours, the reaction solution $(0.4 \mathrm{~mL})$ was mixed with $\mathrm{C}_{6} \mathrm{D}_{6}(0.1 \mathrm{~mL})$. The liquid was analyzed by ${ }^{29} \mathrm{Si} \mathrm{NMR}$ (DEPT) (79 MHz) measurement. We set a peak of hexamethyldisilane as internal standard (-19.5 ppm).

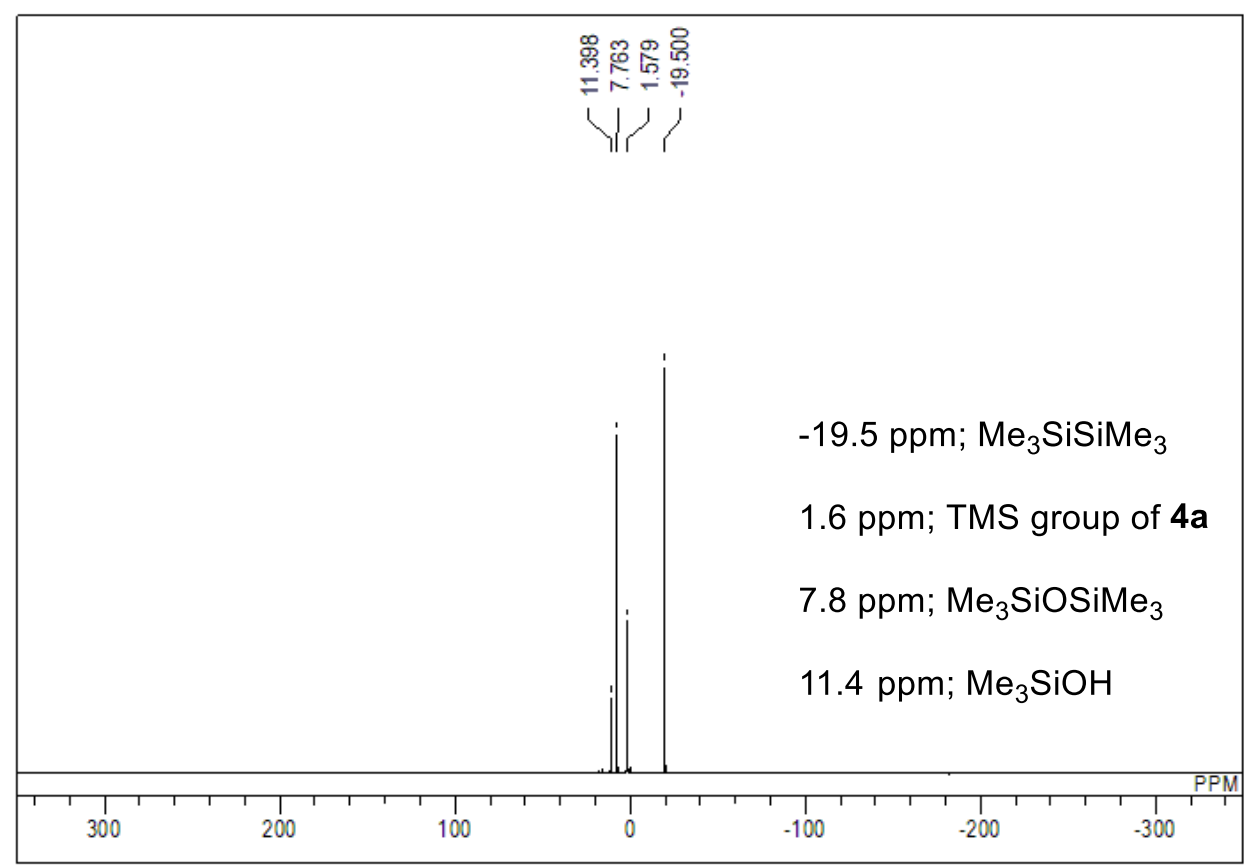

The ${ }^{29} \mathrm{Si}$ (DEPT) NMR chart suggests that $\mathrm{Me}_{3} \mathrm{SiOSiMe}_{3}$ (7.8 ppm) along TMSOH (11.4 ppm) were generated through the reaction. 


\section{Time Course}

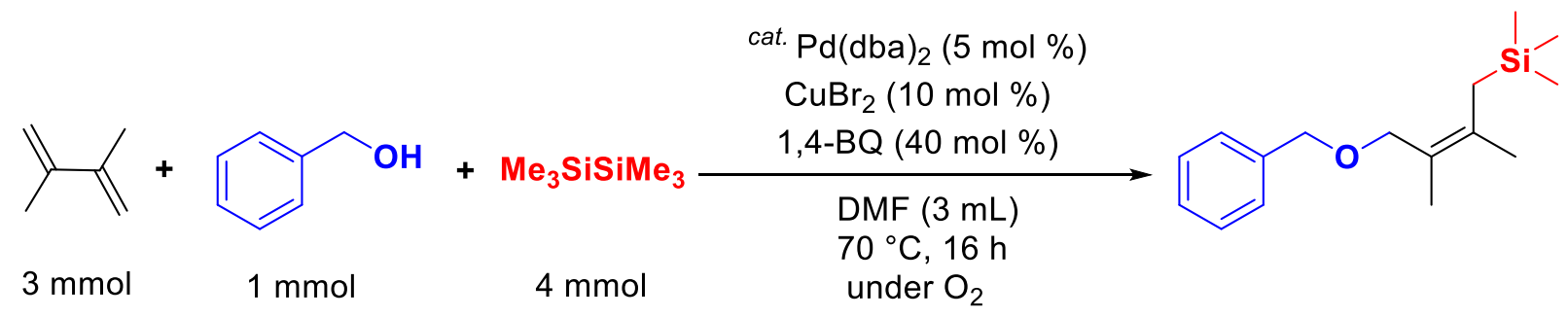

$1 \mathrm{a}$

2a

3a

$4 a$

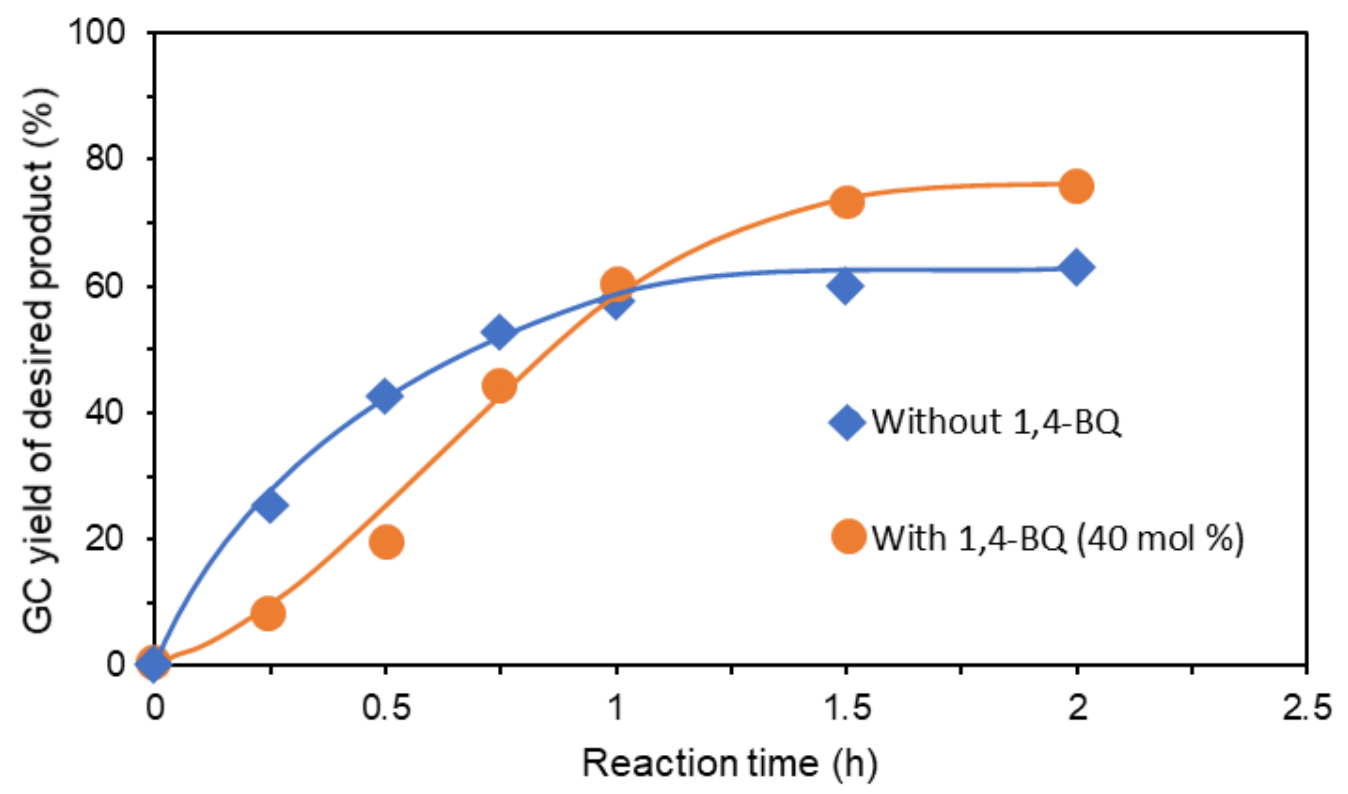

Figure S3. Dependence of 4a's yield over the course of the reaction with and without 1,4-BQ. 


\section{References}

[1] Matsuda, Y.; Tsuji, Y.; Fujihara, T. Cu-Catalyzed Three-Component Coupling Reactions Using Nitriles, 1,3-Dienes and Silylboranes. Chem. Commun. 2020, 56, 4648-4651.

[2] Yasukawa, N.; Yokoyama, H.; Masuda, M.; Monguchi, Y.; Sajiki, H.; Sawama, Y. HighlyFunctionalized Arene Synthesis Based on Palladium on Carbon-Catalyzed Aqueous Dehydrogenation of Cyclohexadienes and Cyclohexenes. Green Chem. 2018, 20, 1213-1217. 
7. NMR Data

4a ${ }^{1} \mathrm{H}$ NMR (400 MHz; $\left.\mathrm{CDCl}_{3}\right)$ :

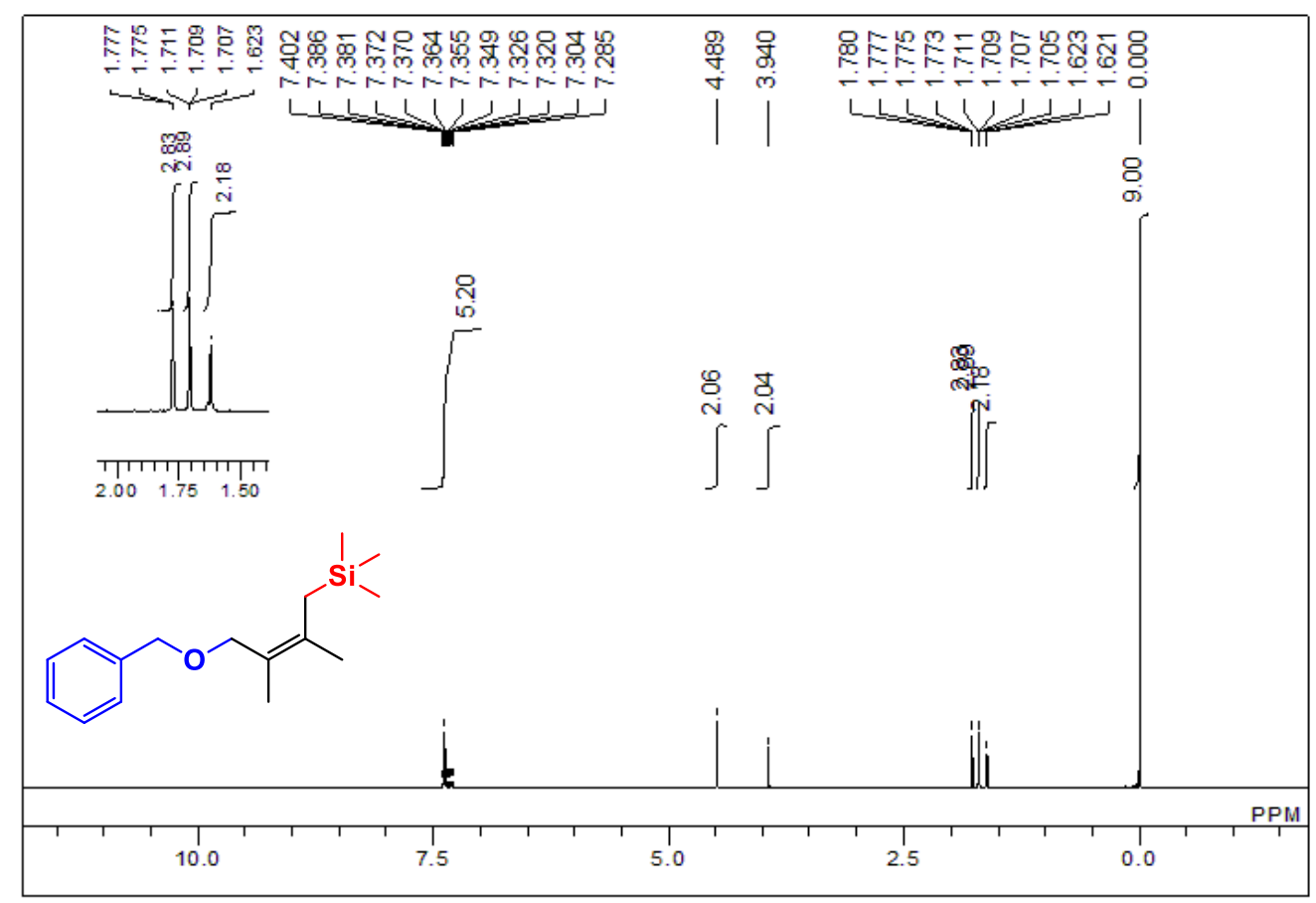

4a ${ }^{13} \mathrm{C}$ NMR (100 MHz; $\left.\mathrm{CDCl}_{3}\right)$ :

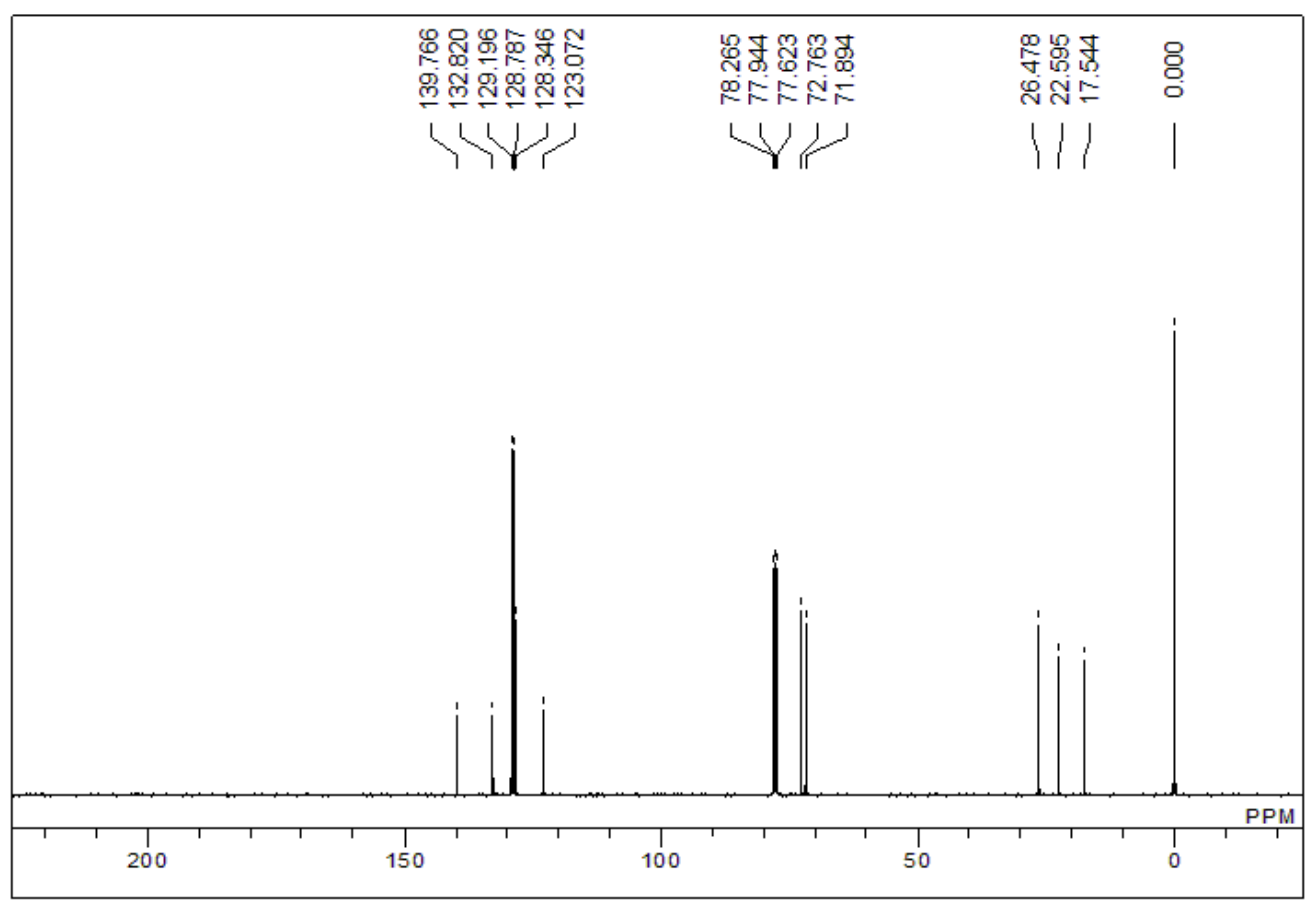


4a $\mathrm{NOE}\left(400 \mathrm{MHz} ; \mathrm{CDCl}_{3}\right)$ :
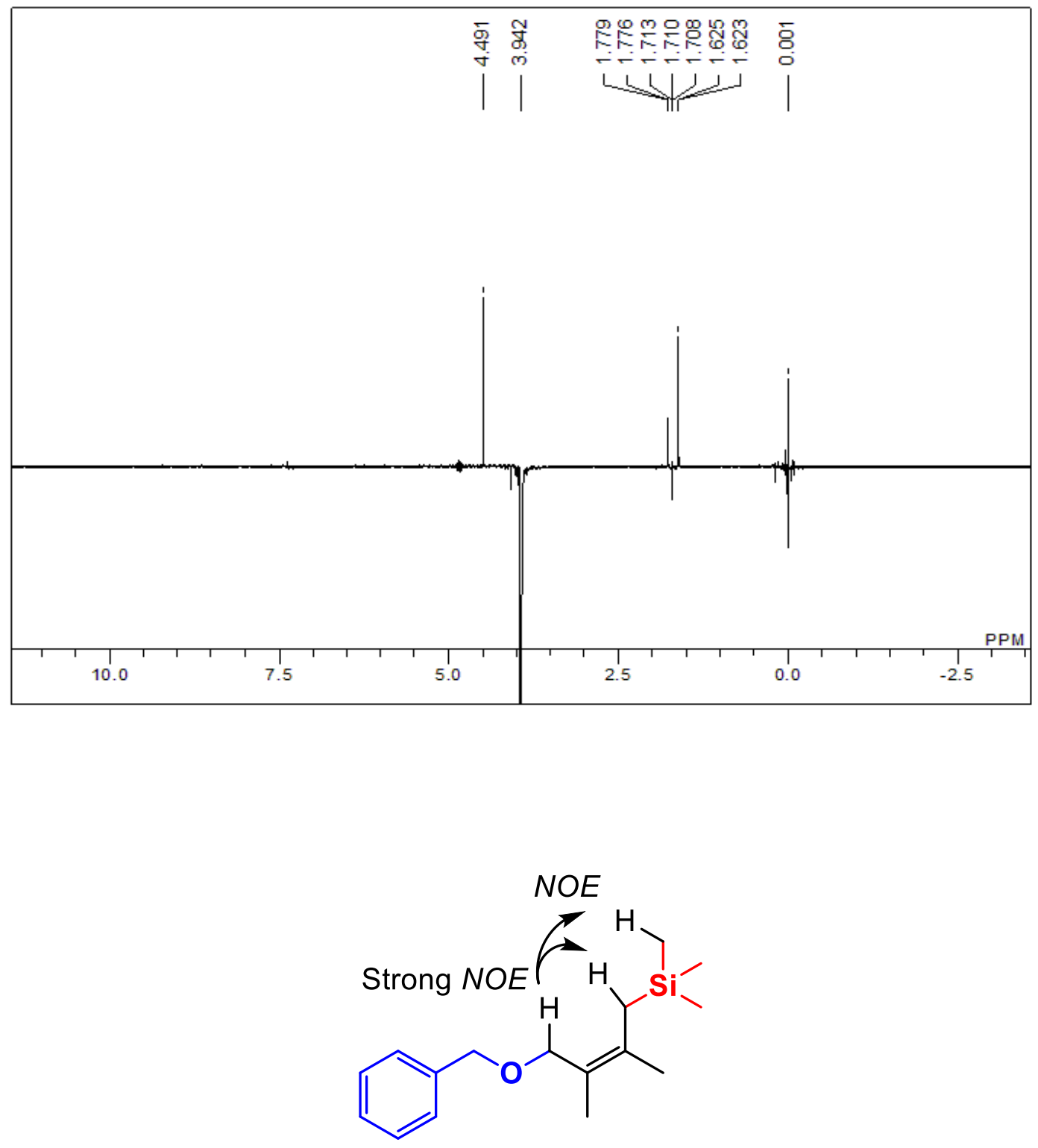

4a: Irradiation at the methylene proton-bearing ether group $(\delta=3.94)$ caused a strong enhancement of the methylene proton $(\delta=1.62)$. 
4a- $d_{7}{ }^{1} \mathrm{H}$ NMR (400 MHz; $\mathrm{CDCl}_{3}$ ):

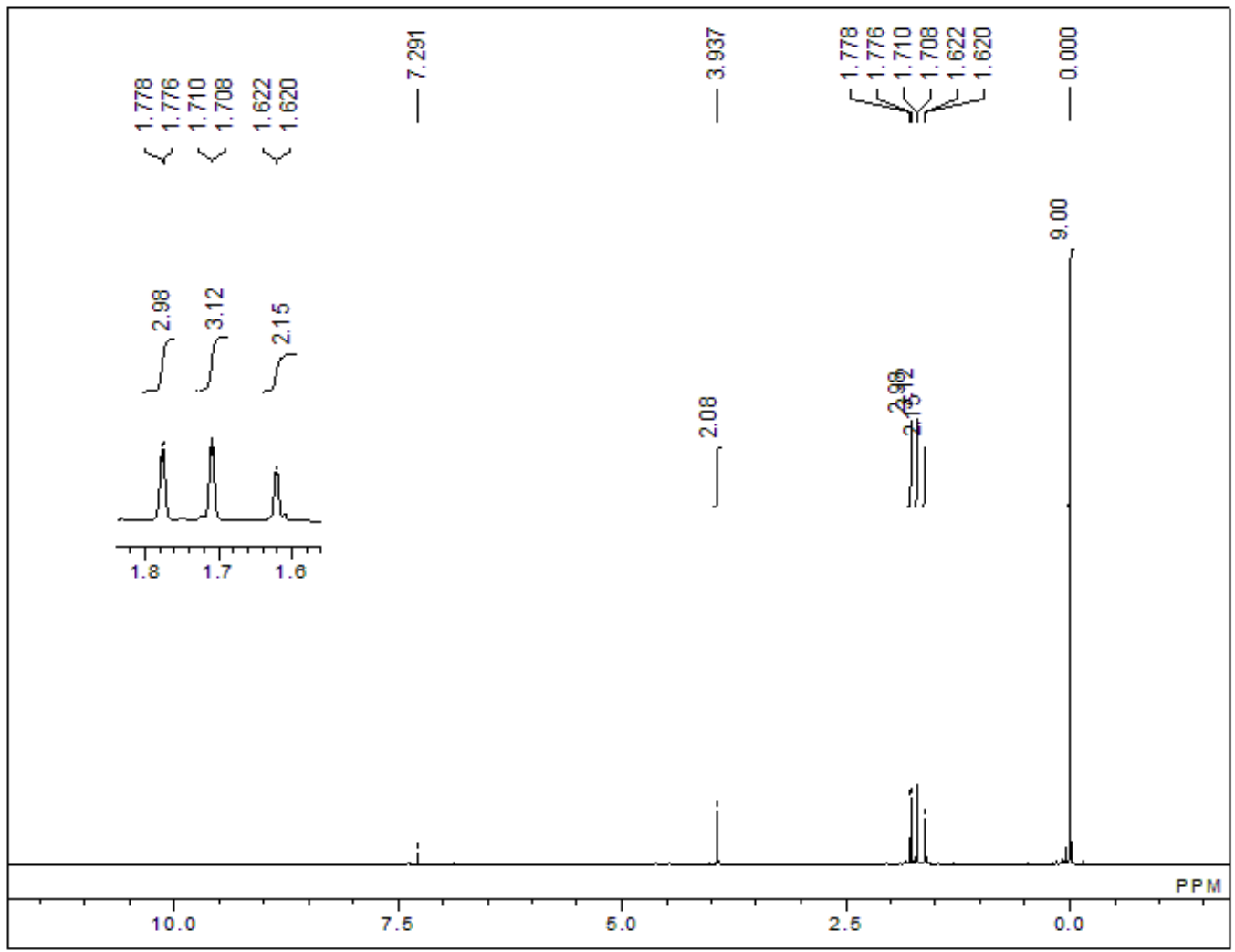

4a- $d{ }^{13} \mathrm{C}$ NMR (100 MHz; $\left.\mathrm{CDCl}_{3}\right)$ :

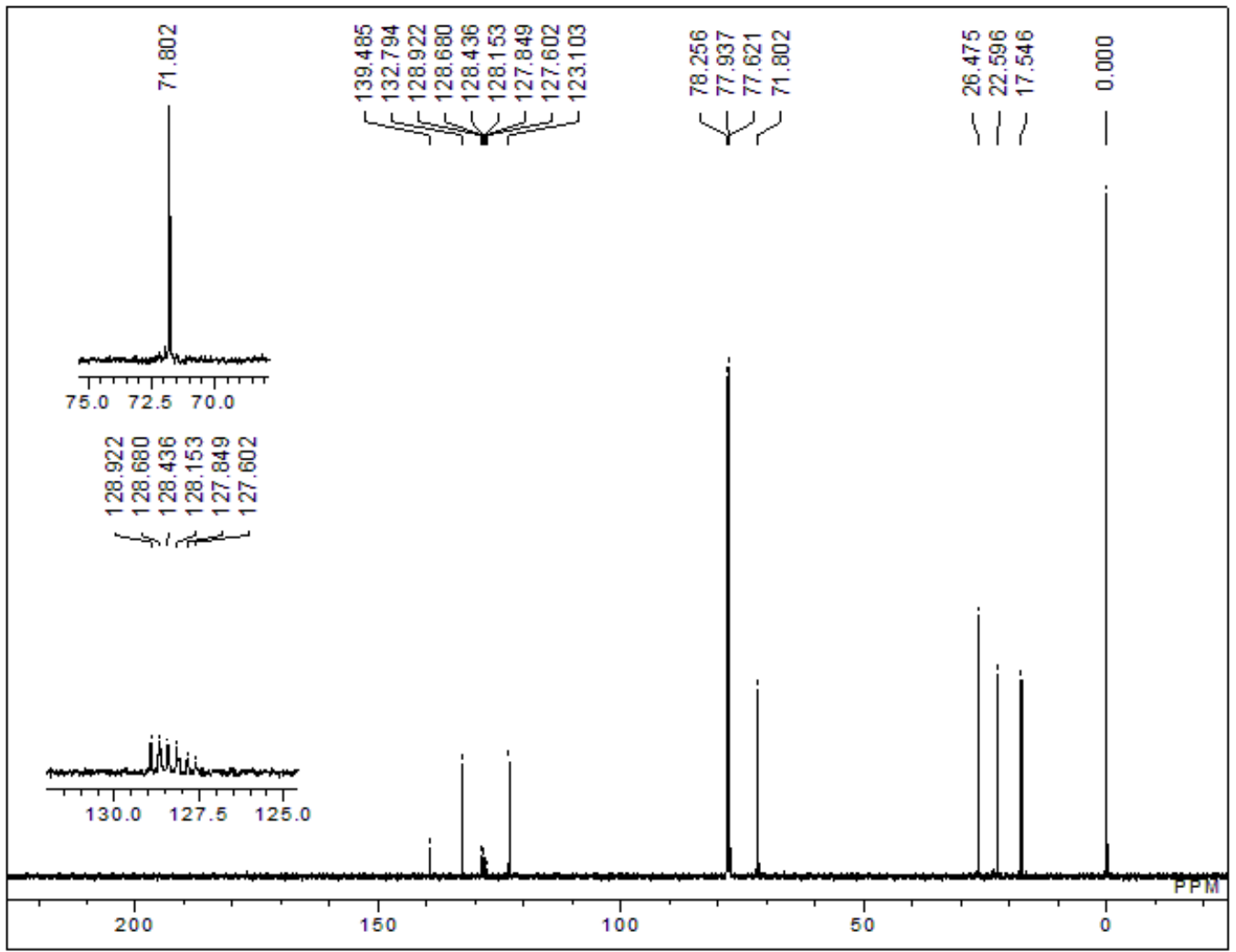


4b ${ }^{1} \mathrm{H}$ NMR (400 MHz; $\left.\mathrm{CDCl}_{3}\right)$ :

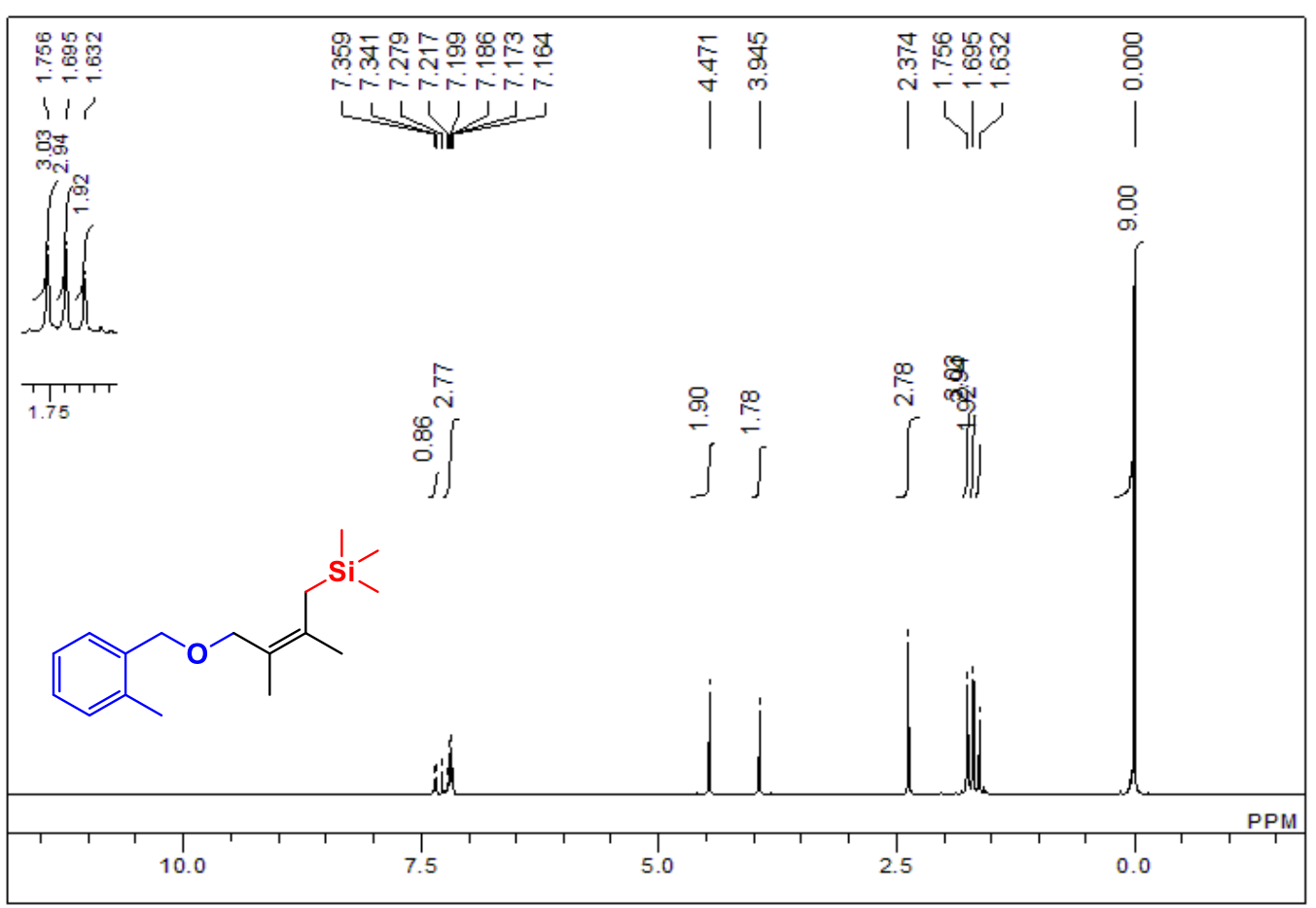

4b ${ }^{13} \mathrm{C}$ NMR (100 MHz; $\left.\mathrm{CDCl}_{3}\right)$ :

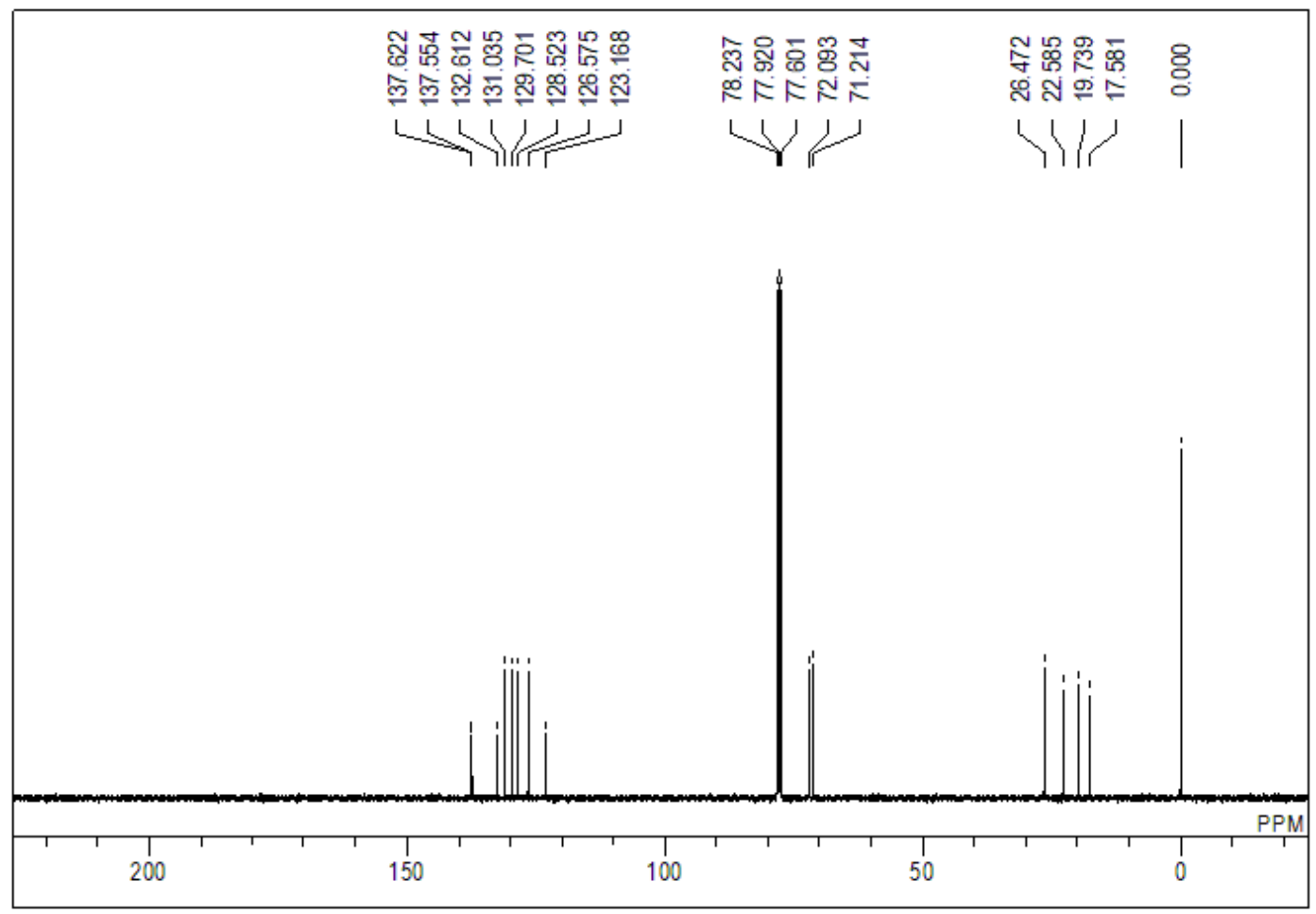


4b $\mathrm{NOE}$ (400 MHz; $\left.\mathrm{CDCl}_{3}\right)$ :
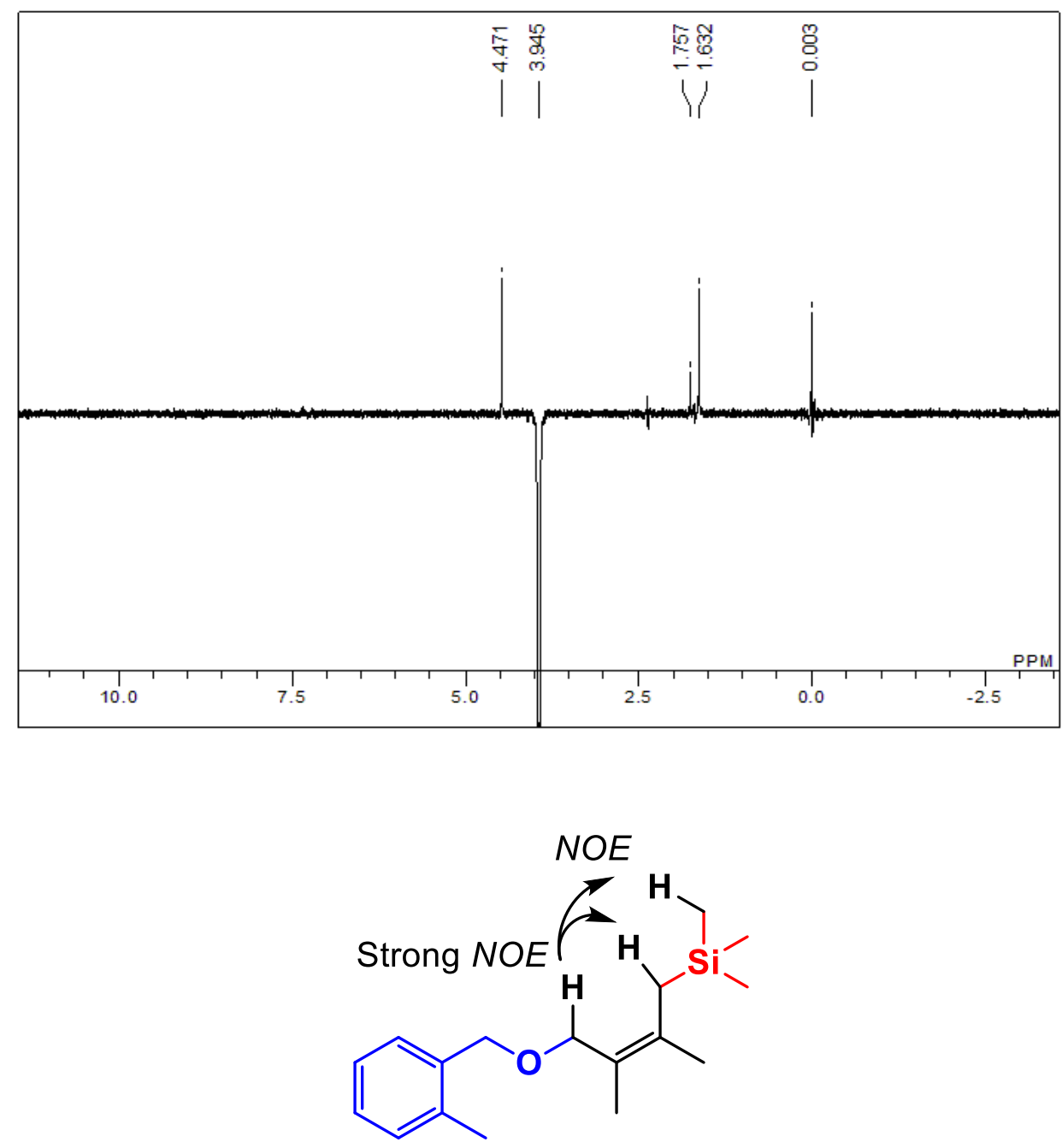

4b: Irradiation at the methylene proton-bearing ether group $(\delta=3.95)$ caused strong enhancement of the methylene proton $(\delta=1.63)$. 
4c ${ }^{1} \mathrm{H}$ NMR (400 MHz; $\mathrm{CDCl}_{3}$ ):

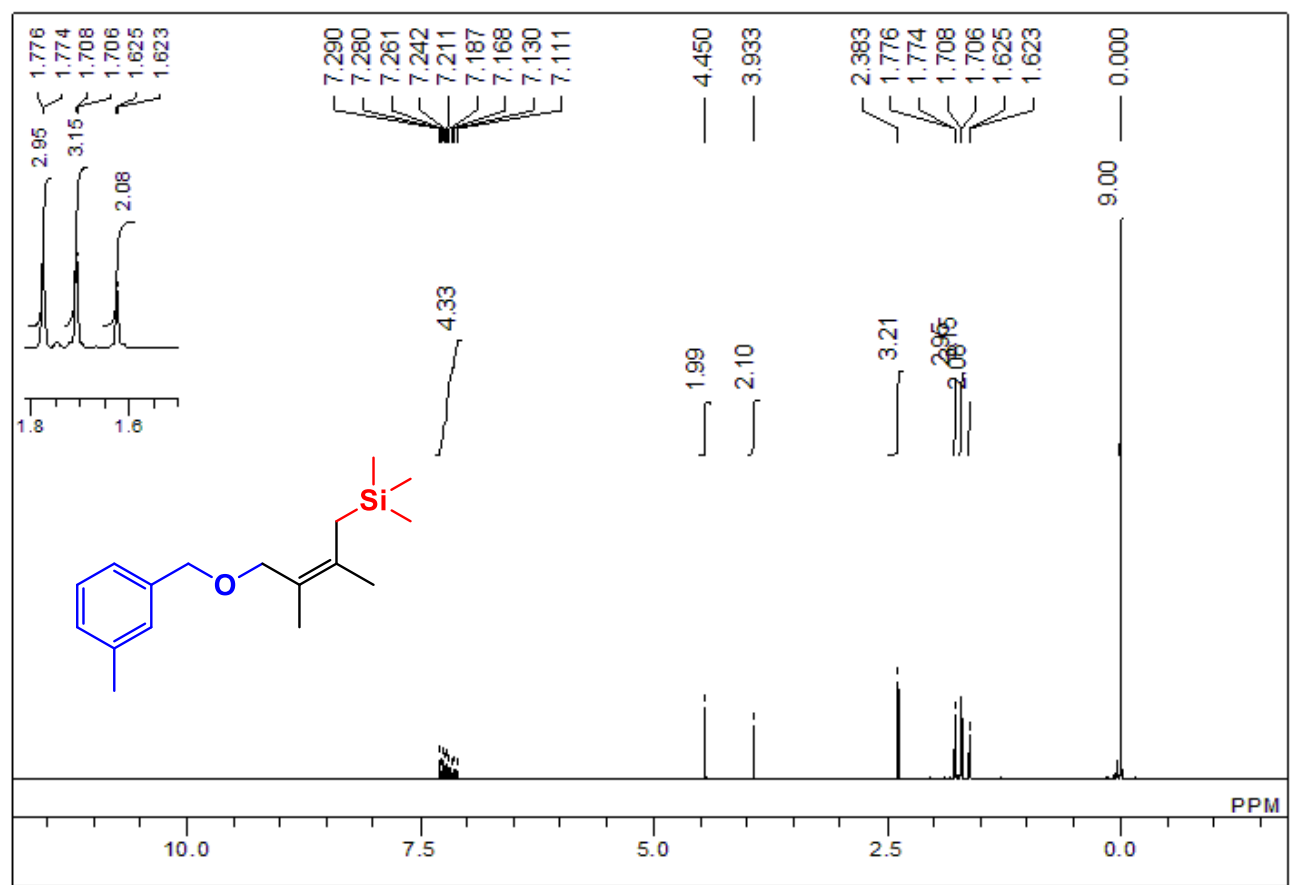

4c ${ }^{13} \mathrm{C}$ NMR (100 MHz; $\left.\mathrm{CDCl}_{3}\right)$ :

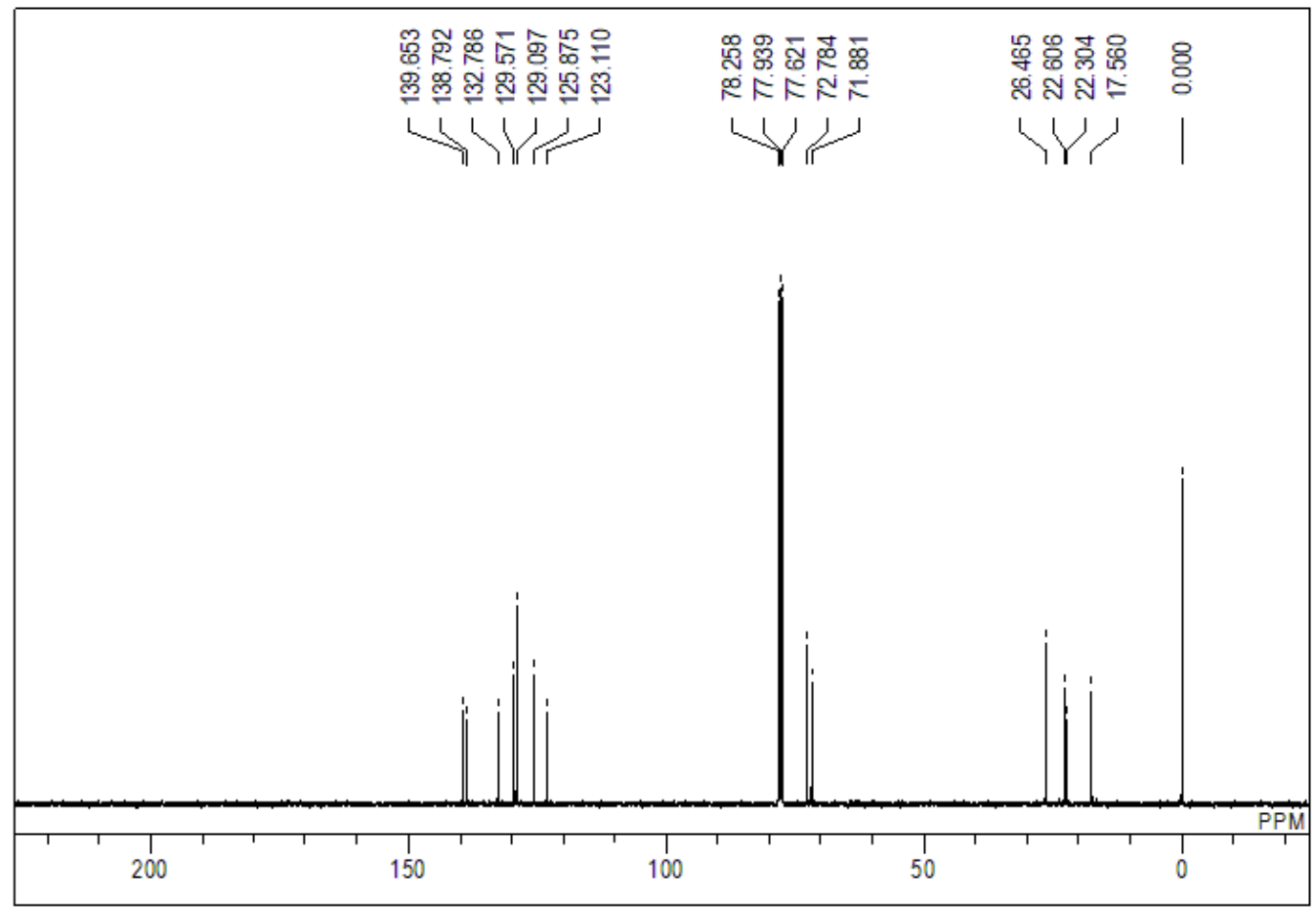


4c $\mathrm{NOE}\left(400 \mathrm{MHz} ; \mathrm{CDCl}_{3}\right)$ :
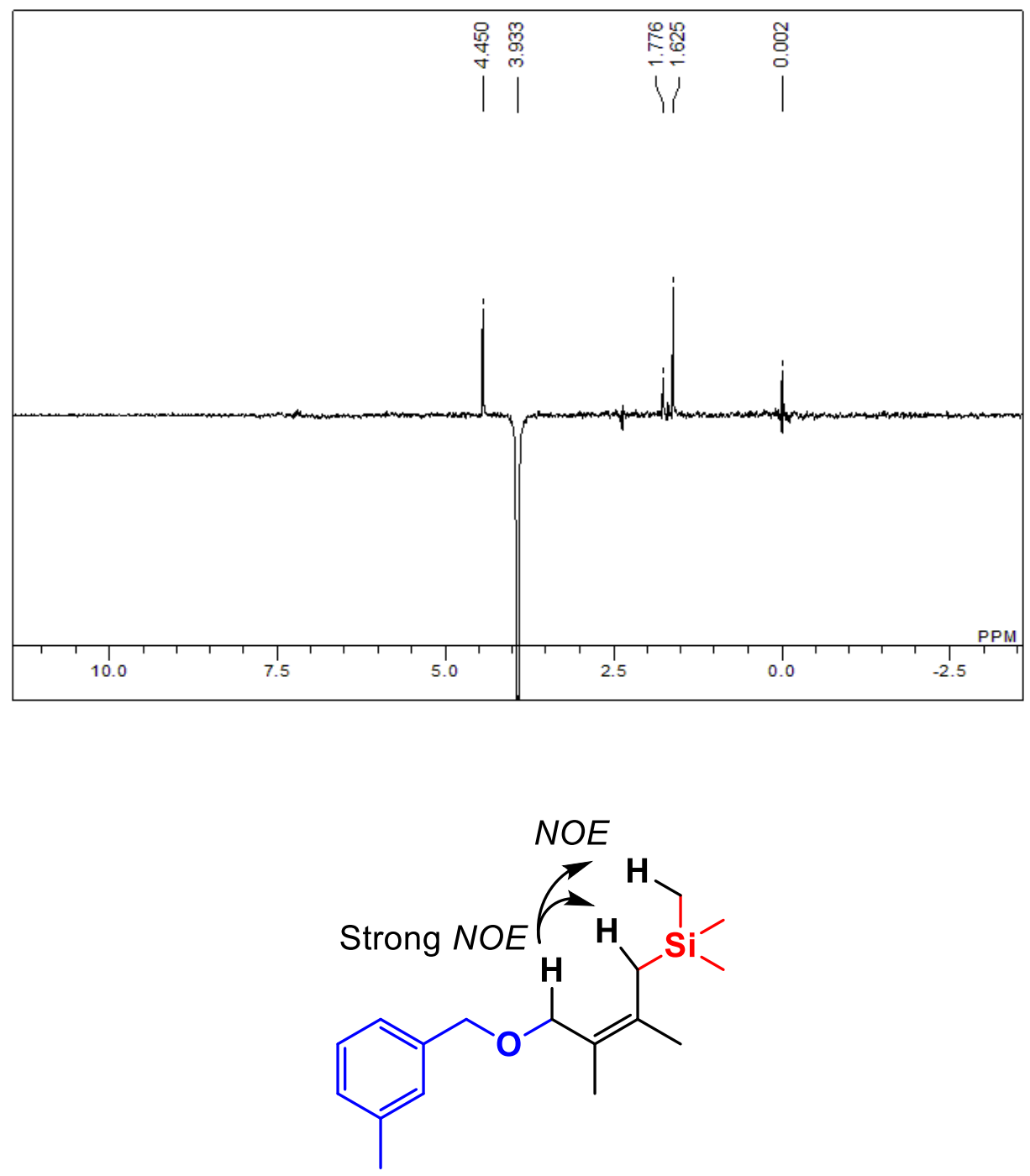

4c: Irradiation at the methylene proton-bearing ether group $(\delta=3.93)$ caused strong enhancement of the methylene proton $(\delta=1.63)$. 
4d ${ }^{1} \mathrm{H}$ NMR (400 MHz; $\left.\mathrm{CDCl}_{3}\right)$ :

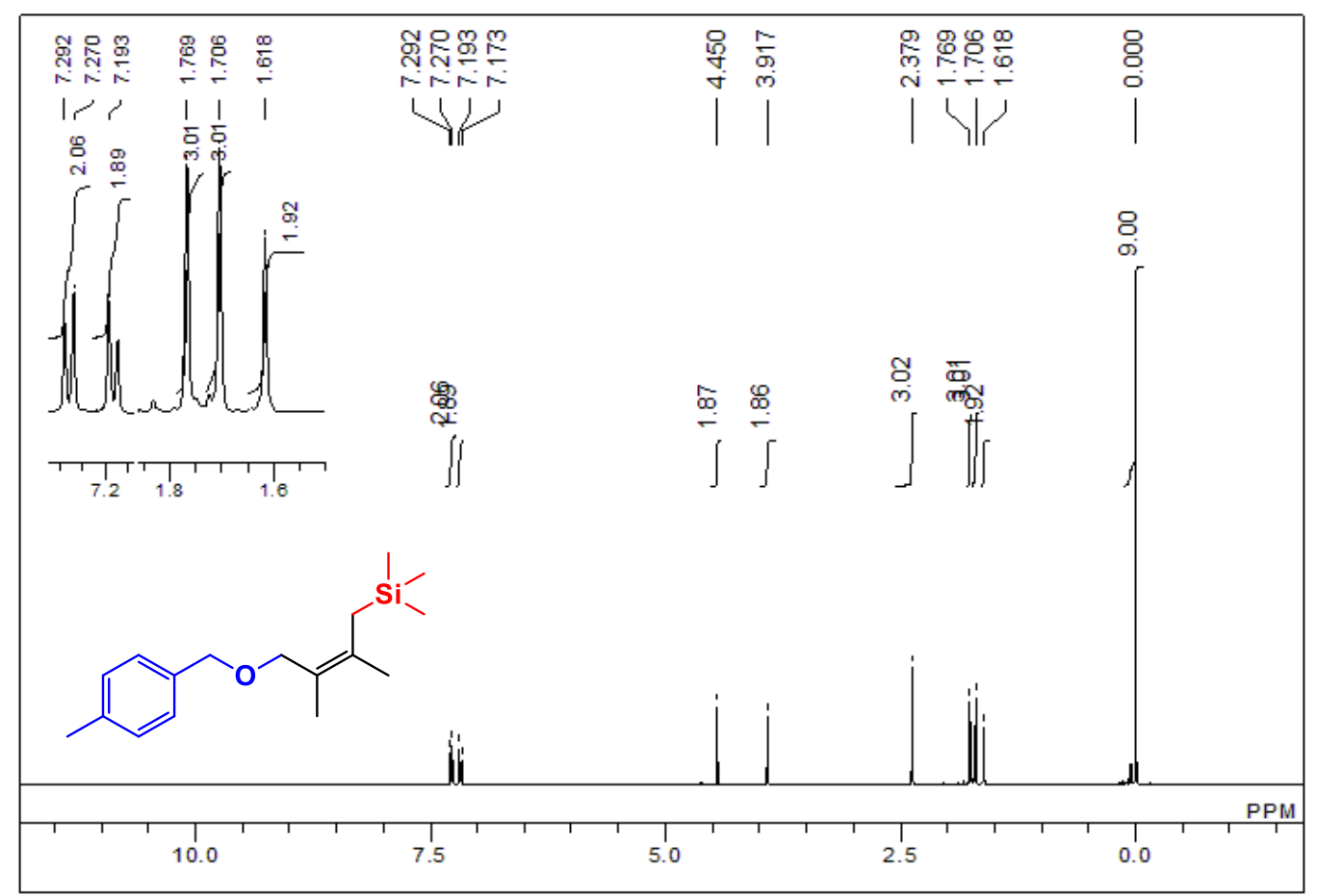

4d ${ }^{13} \mathrm{C}$ NMR (100 MHz; $\left.\mathrm{CDCl}_{3}\right)$ :

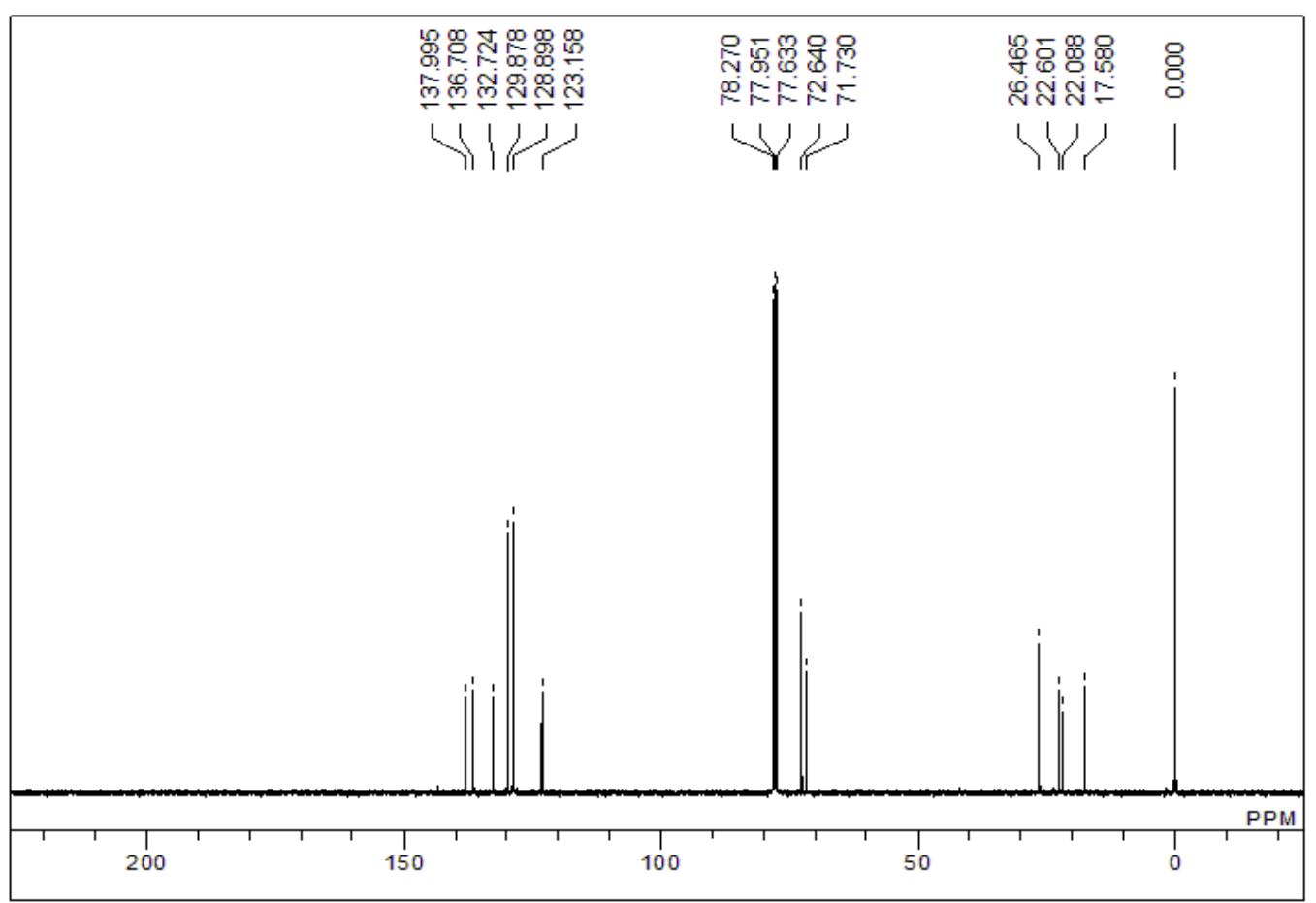


4d NOE (400 MHz; $\left.\mathrm{CDCl}_{3}\right)$ :
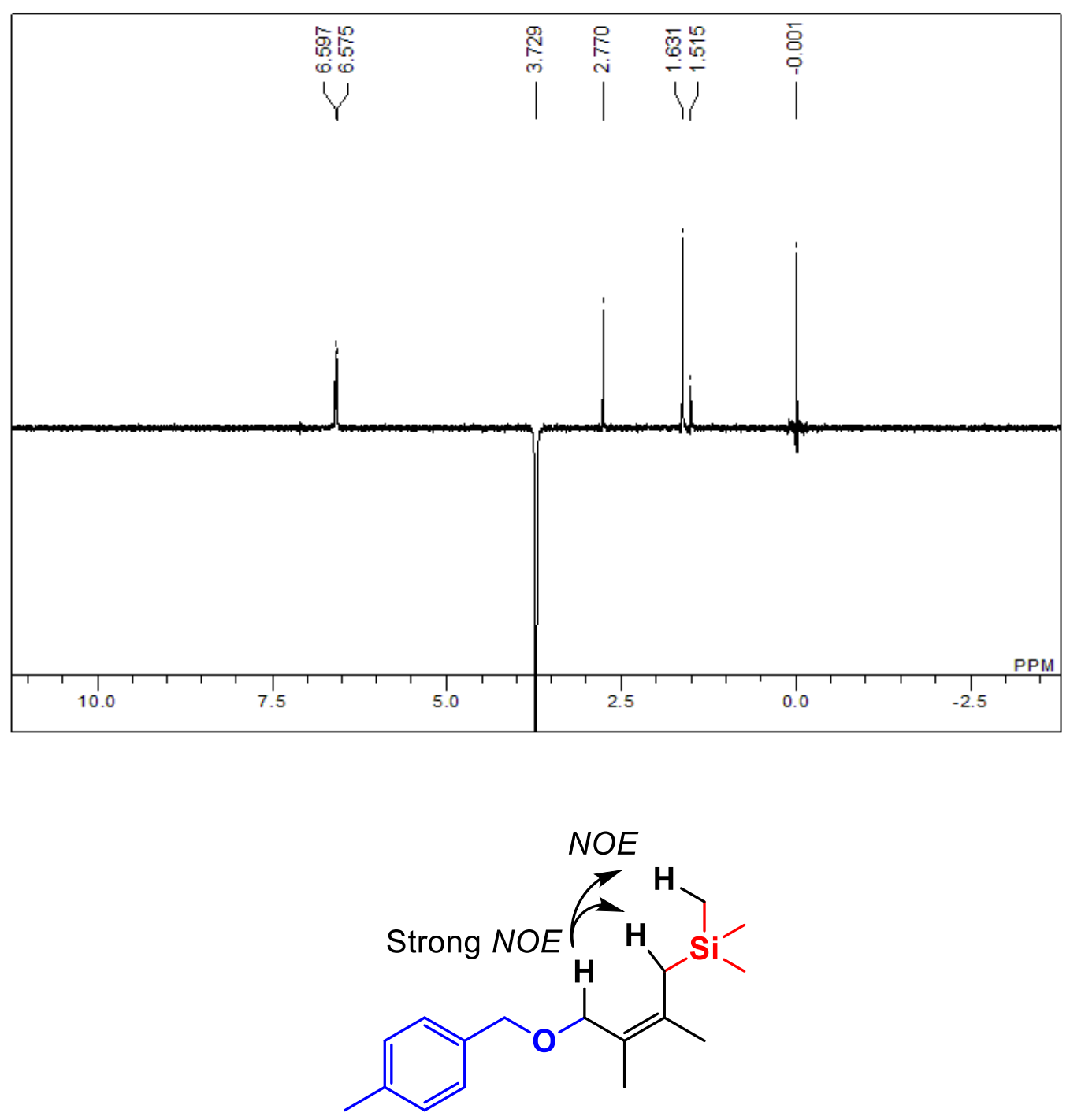

4d: Irradiation at the methylene proton-bearing ether group $(\delta=3.73)$ caused strong enhancement of the methylene proton $(\delta=1.63)$. 
4e ${ }^{1} \mathrm{H}$ NMR (400 MHz; $\mathrm{CDCl}_{3}$ ):

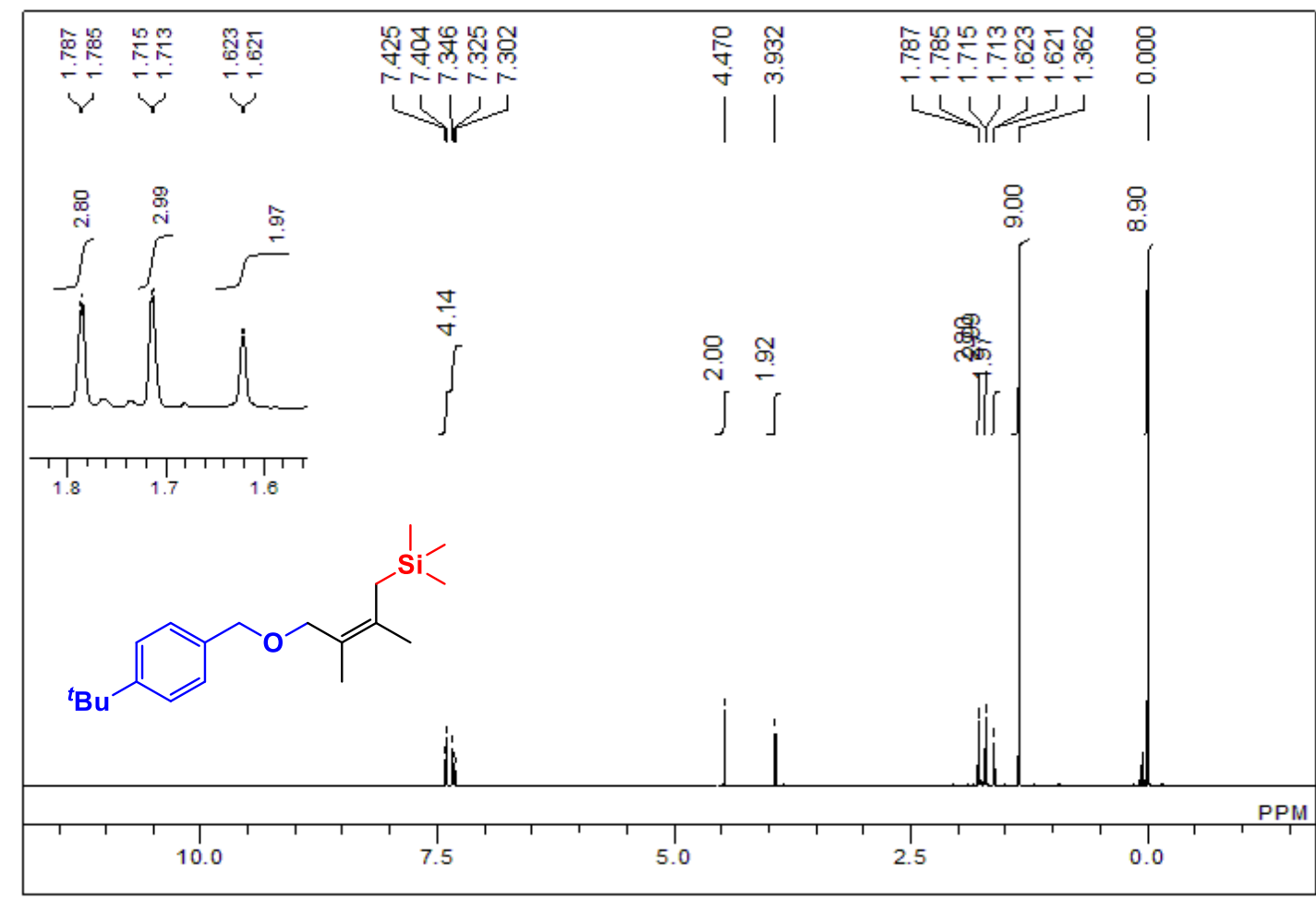

4e ${ }^{13} \mathrm{C}$ NMR (100 MHz; $\left.\mathrm{CDCl}_{3}\right)$ :

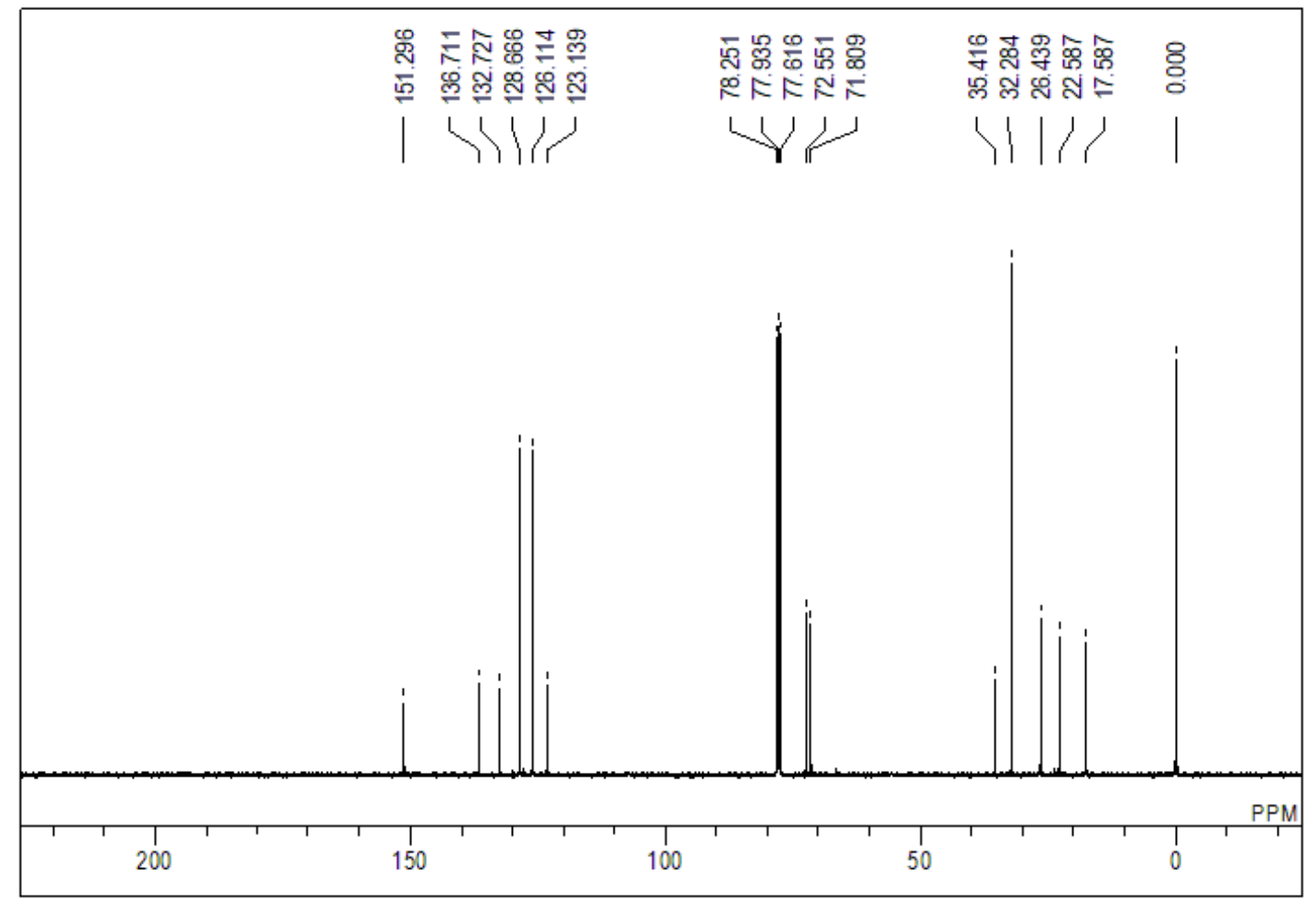


4e $\mathrm{NOE}\left(400 \mathrm{MHz} ; \mathrm{CDCl}_{3}\right)$ :
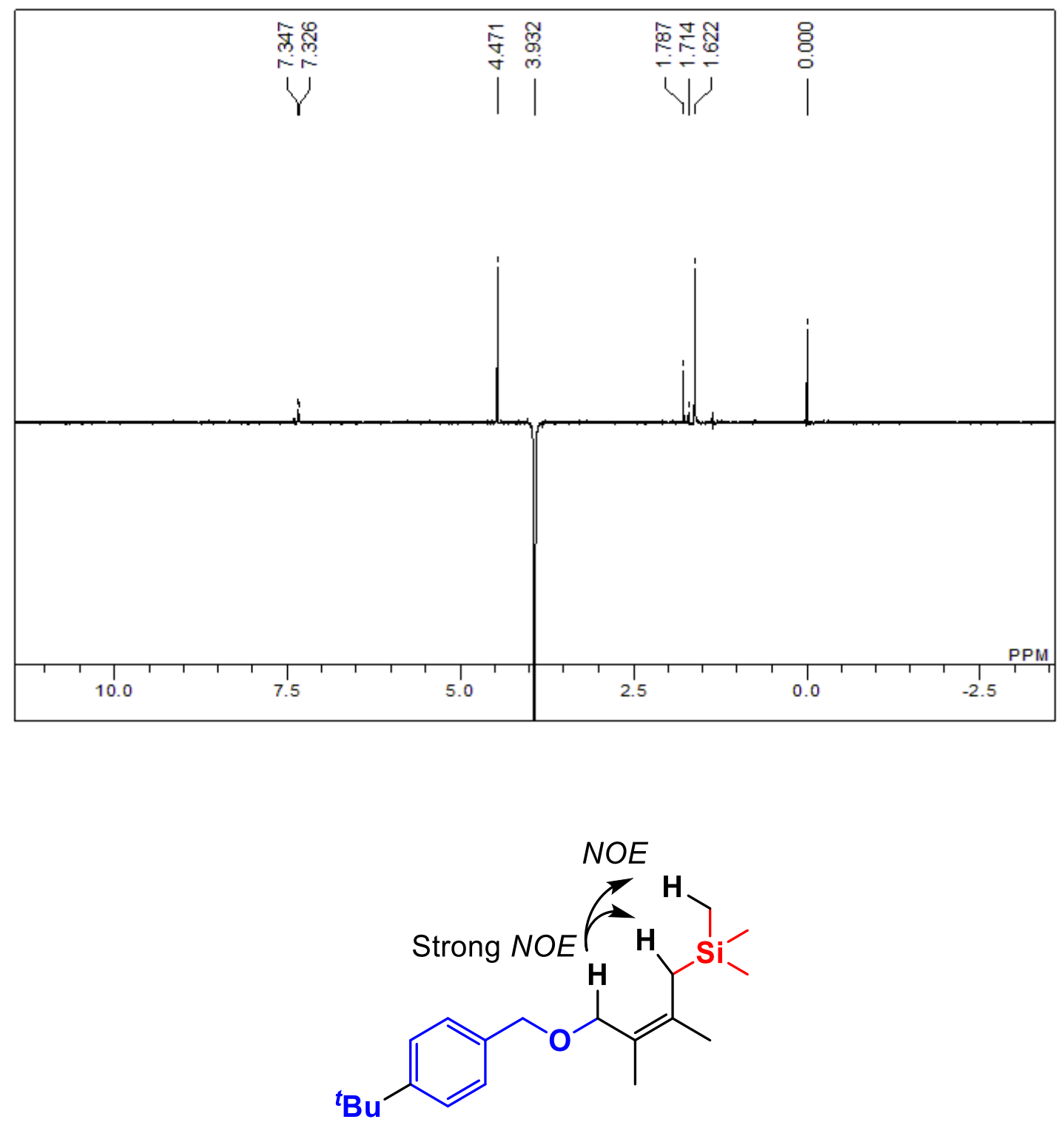

4e: Irradiation at the methylene proton-bearing ether group $(\delta=3.93)$ caused strong enhancement of the methylene proton $(\delta=1.62)$. 
4f ${ }^{1} \mathrm{H}$ NMR (400 MHz; $\left.\mathrm{CDCl}_{3}\right)$ :

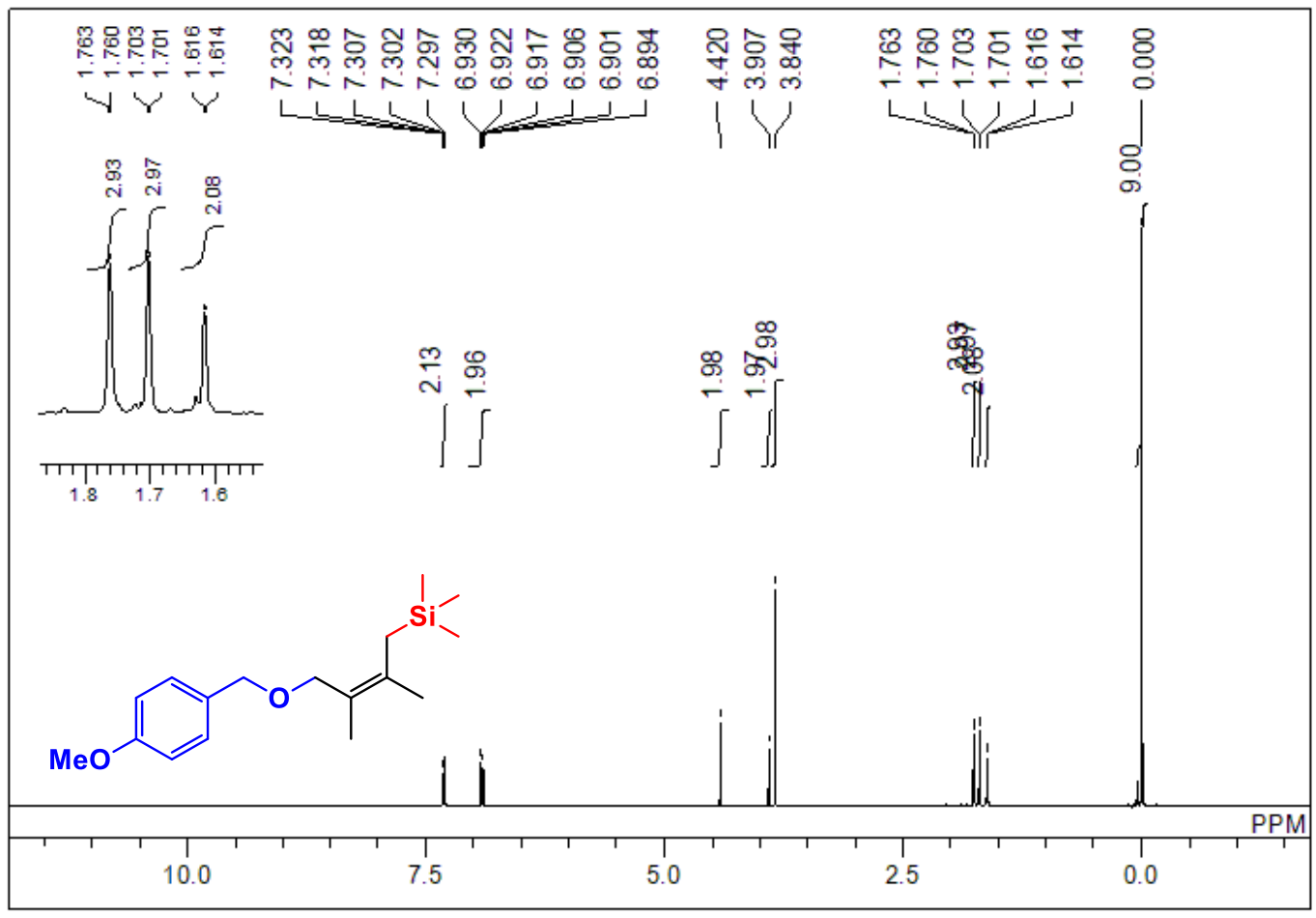

4f ${ }^{13} \mathrm{C}$ NMR (100 MHz; $\left.\mathrm{CDCl}_{3}\right)$ :

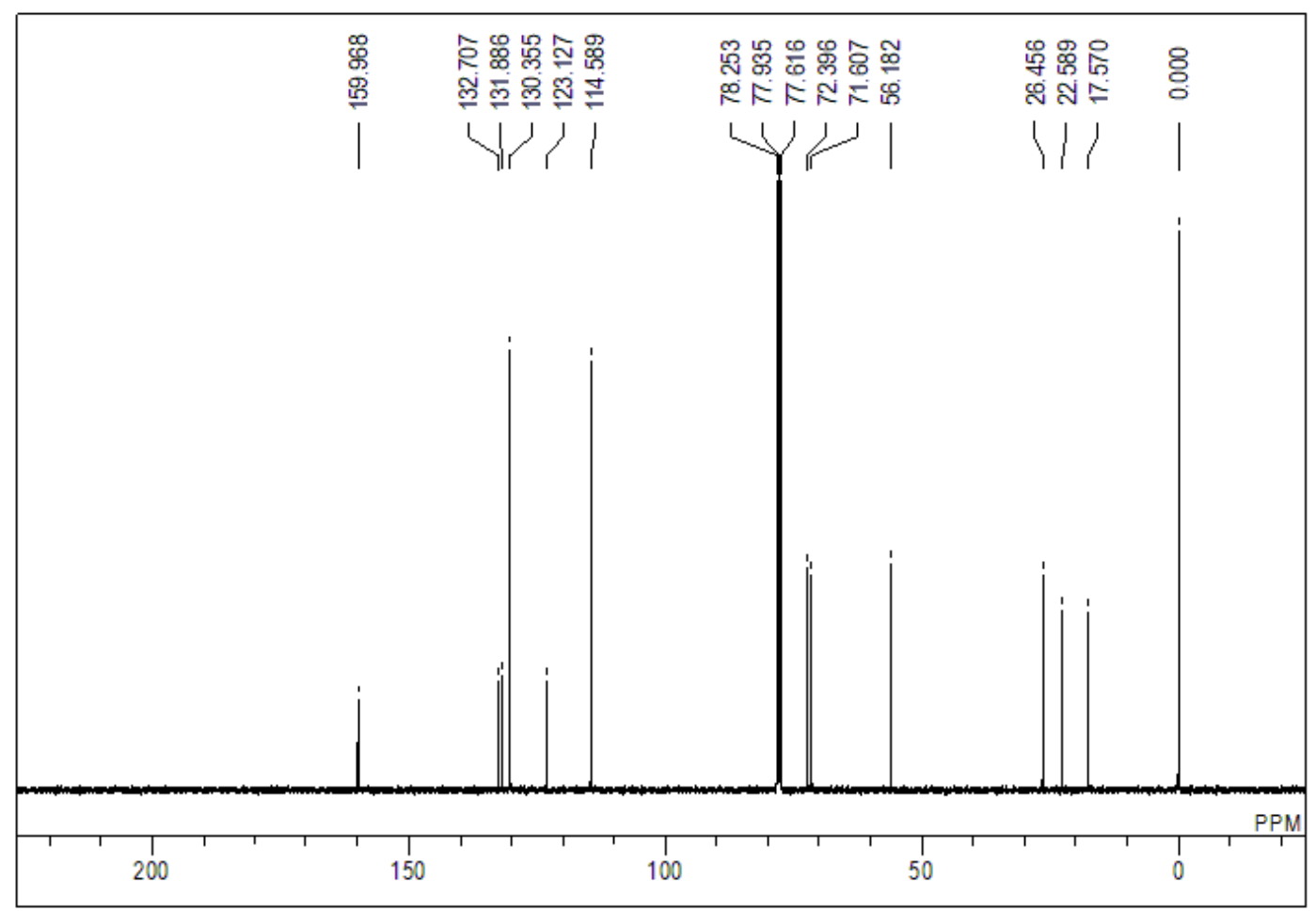


4f $\mathrm{NOE}\left(400 \mathrm{MHz} ; \mathrm{CDCl}_{3}\right)$ :
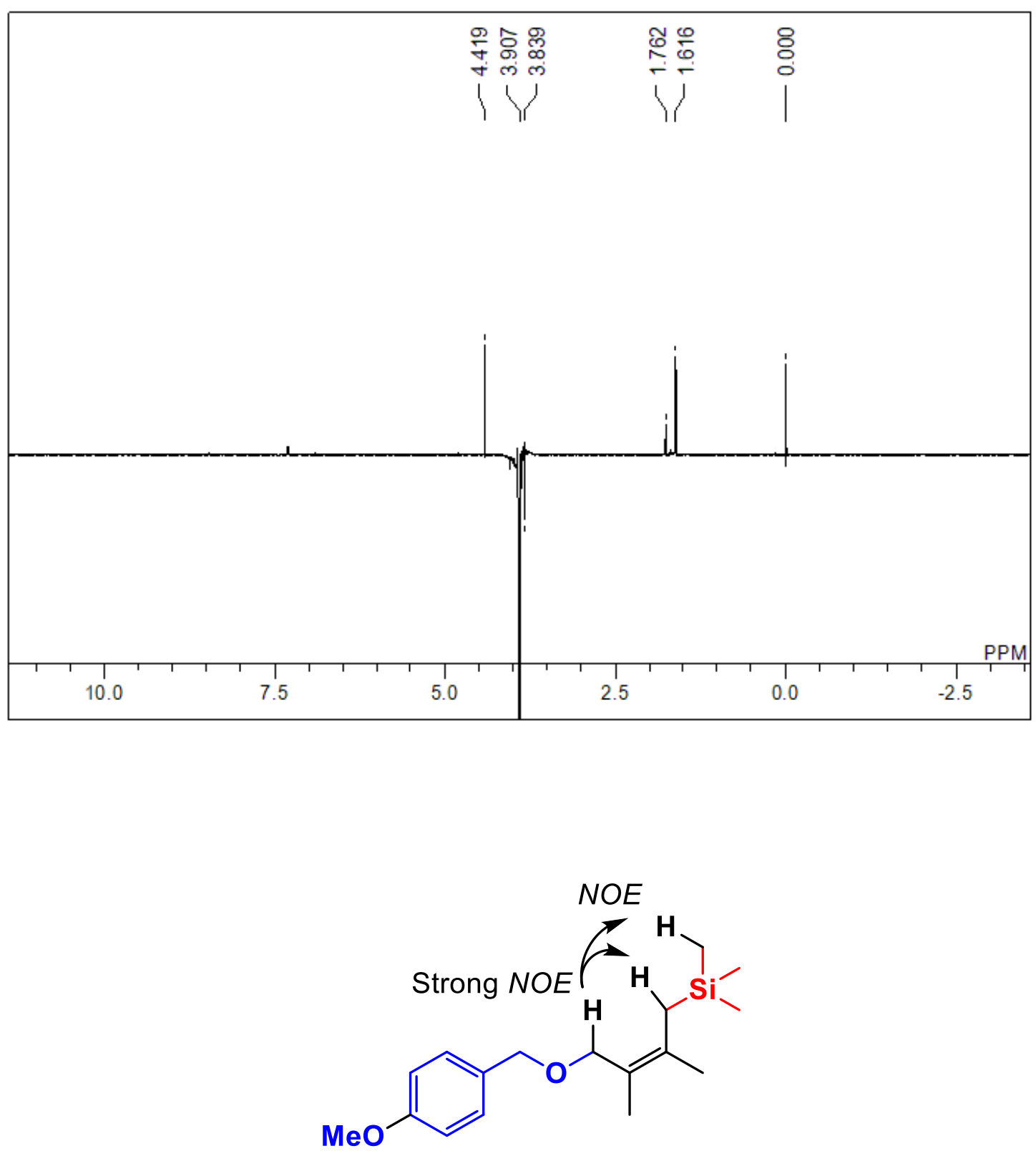

4f: Irradiation at the methylene proton-bearing ether group $(\delta=3.91)$ caused strong enhancement of the methylene proton $(\delta=1.62)$. 
4g ${ }^{1} \mathrm{H}$ NMR (400 MHz; $\left.\mathrm{CDCl}_{3}\right)$ :

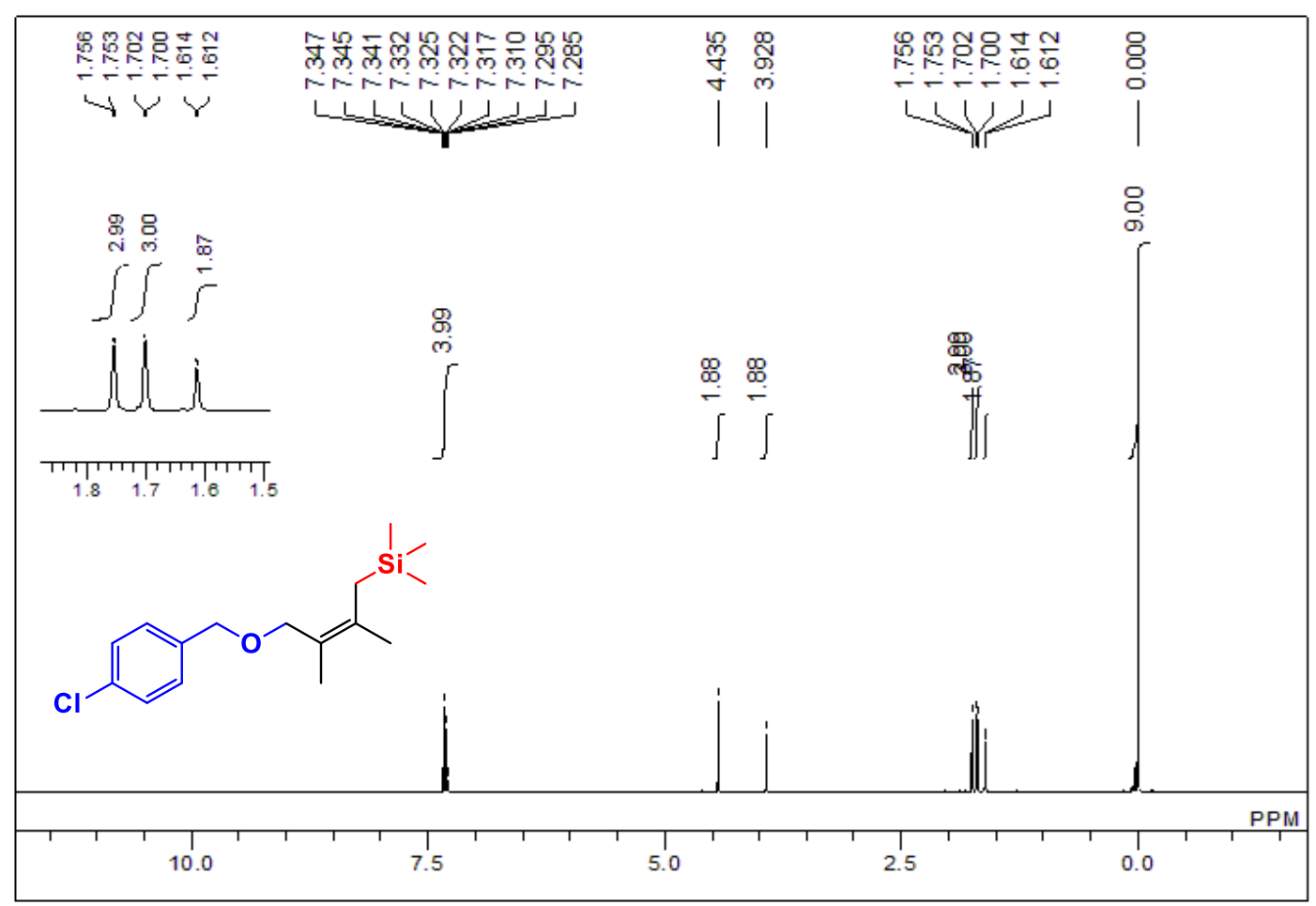

4g ${ }^{13} \mathrm{C}$ NMR (100 MHz; $\left.\mathrm{CDCl}_{3}\right)$ :

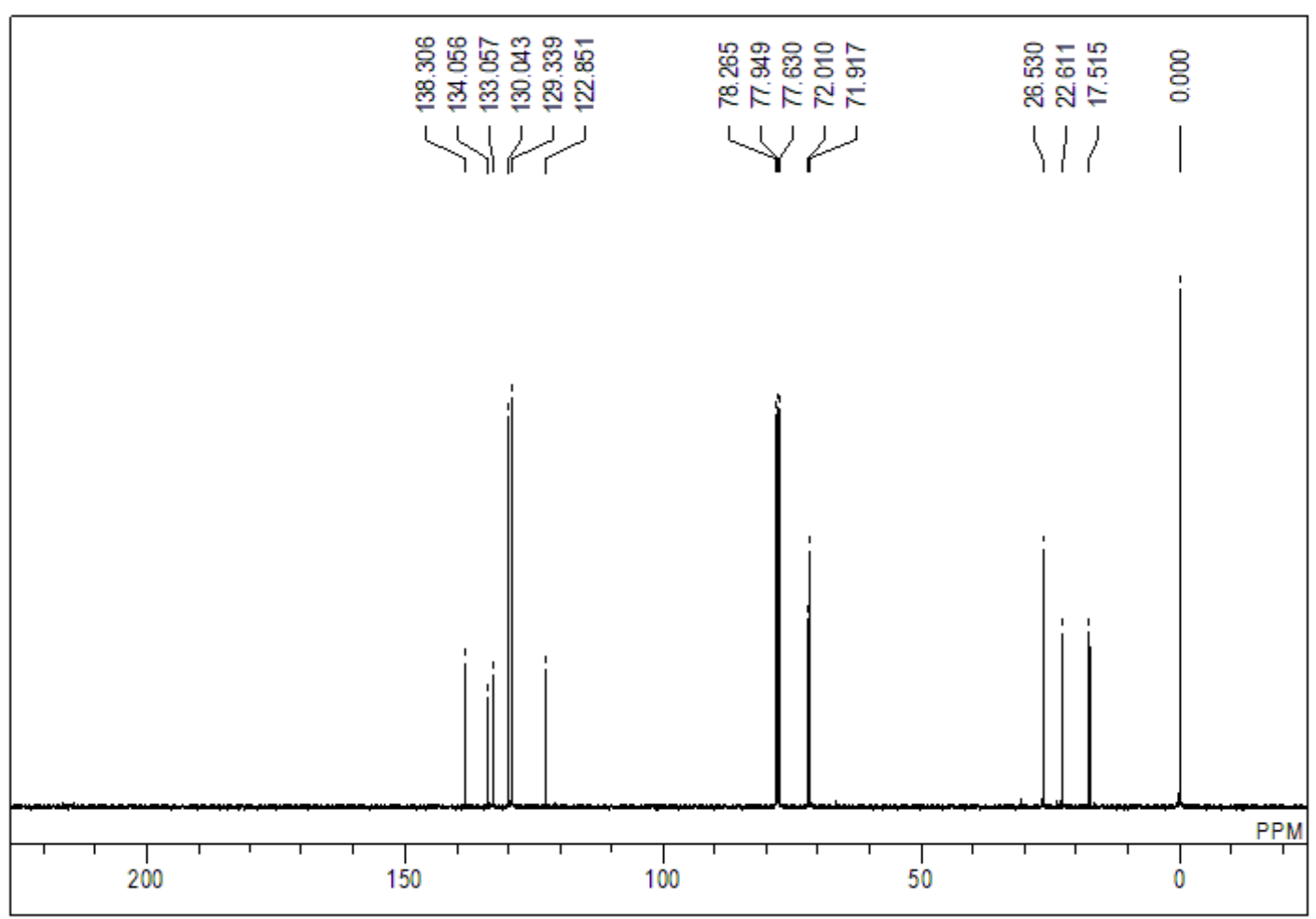


4g NOE (400 MHz; $\left.\mathrm{CDCl}_{3}\right)$ :
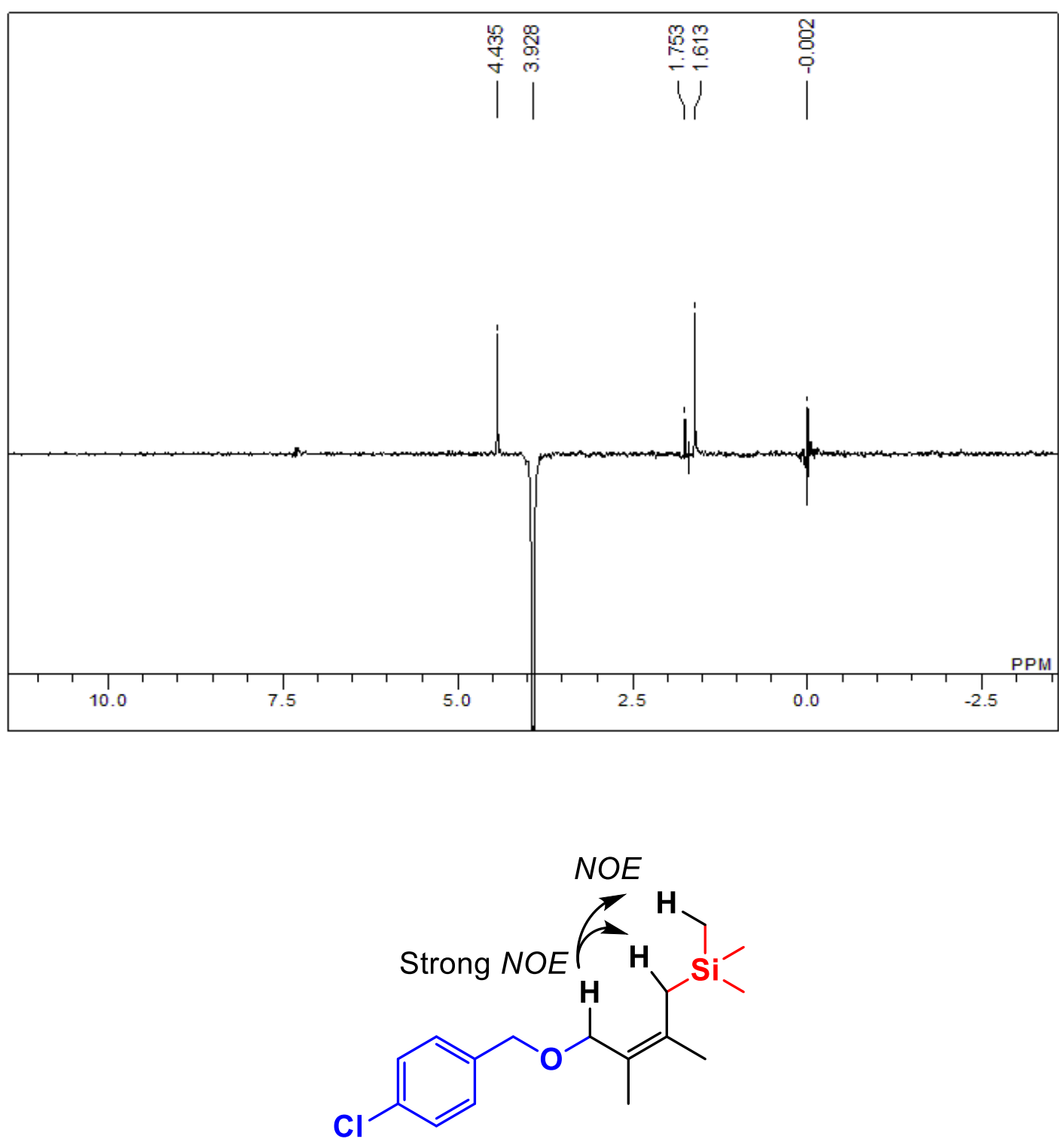

4g: Irradiation at the methylene proton-bearing ether group $(\delta=3.93)$ caused strong enhancement of the methylene proton $(\delta=1.61)$ 
4h ${ }^{1} \mathrm{H}$ NMR (400 MHz; $\left.\mathrm{CDCl}_{3}\right)$ :

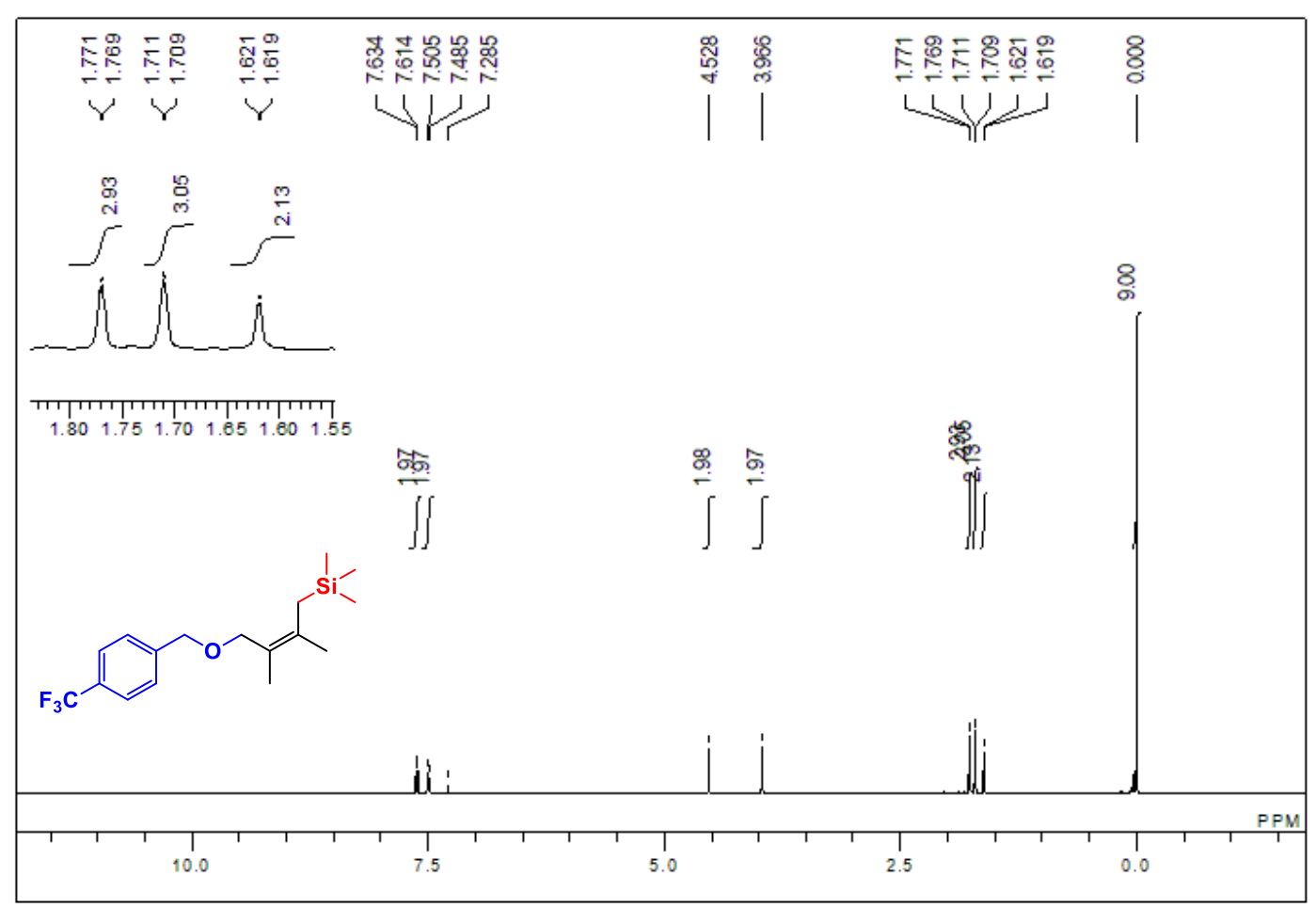

4h ${ }^{13} \mathrm{C}$ NMR (100 MHz; $\left.\mathrm{CDCl}_{3}\right)$ :

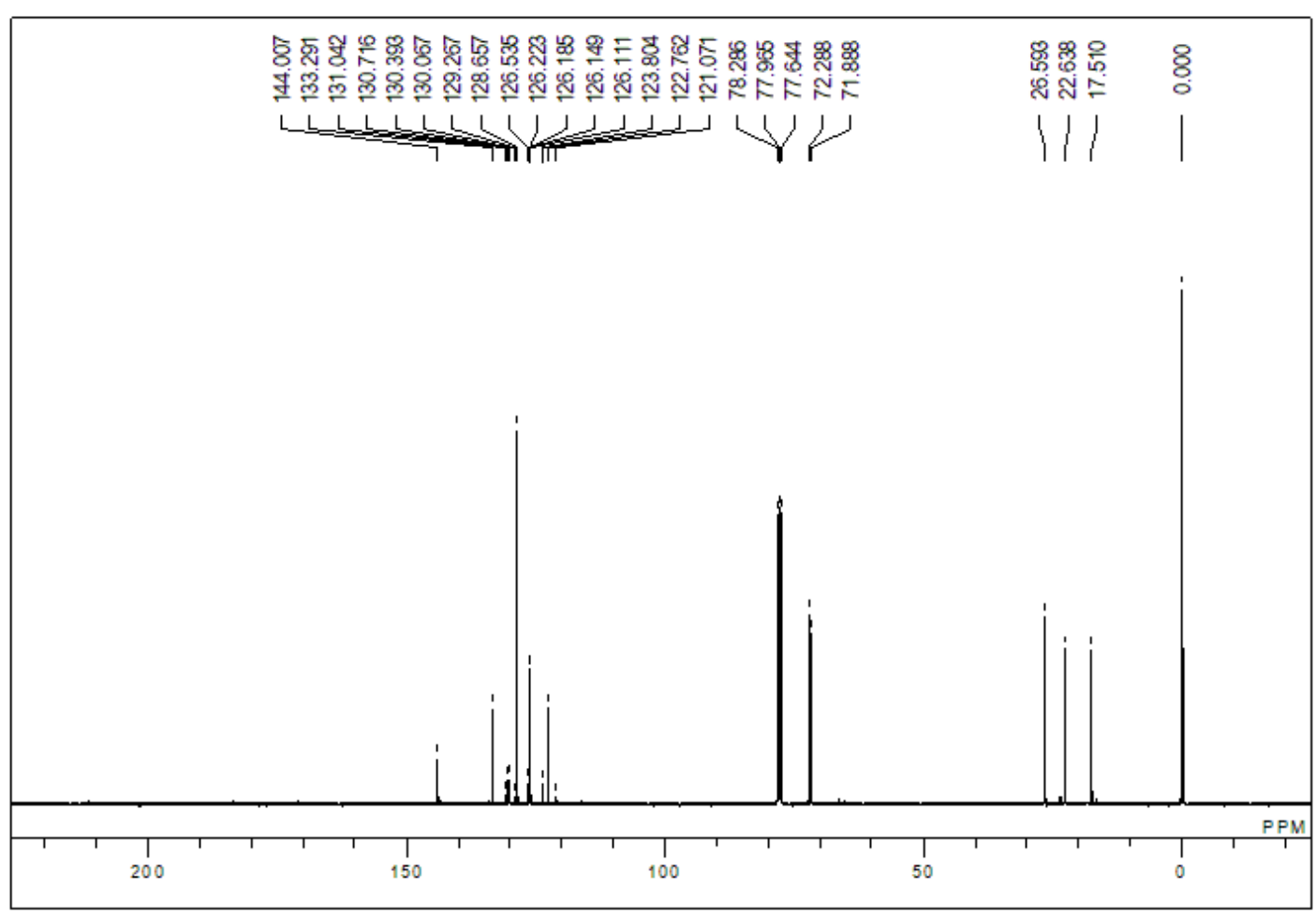


4h $\mathrm{NOE}$ (400 MHz; $\left.\mathrm{CDCl}_{3}\right)$ :
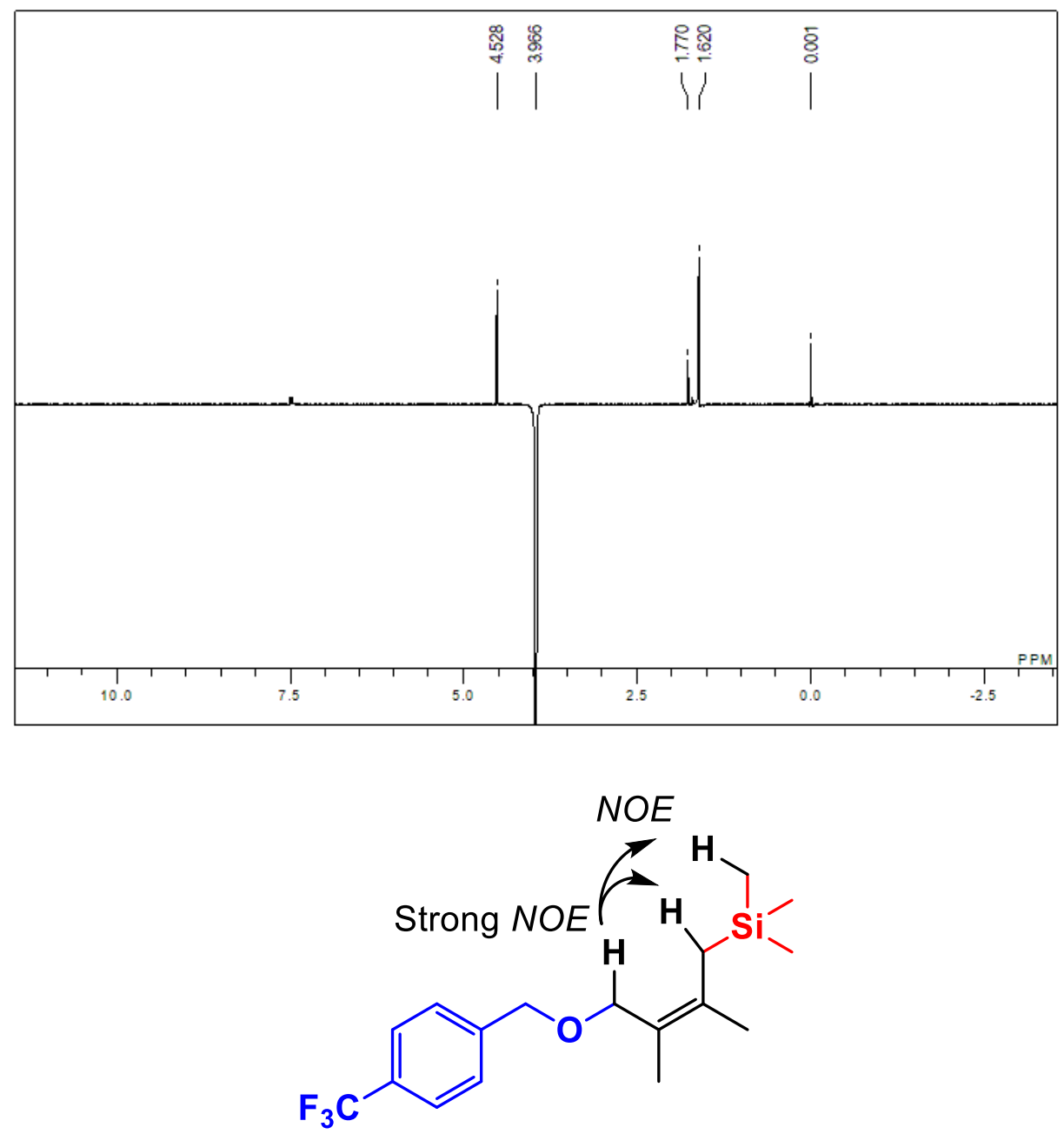

4h: Irradiation at the methylene proton-bearing ether group $(\delta=3.97)$ caused strong enhancement of the methylene proton $(\delta=1.62)$. 
4i ${ }^{1} \mathrm{H}$ NMR (400 MHz; $\left.\mathrm{CDCl}_{3}\right)$ :

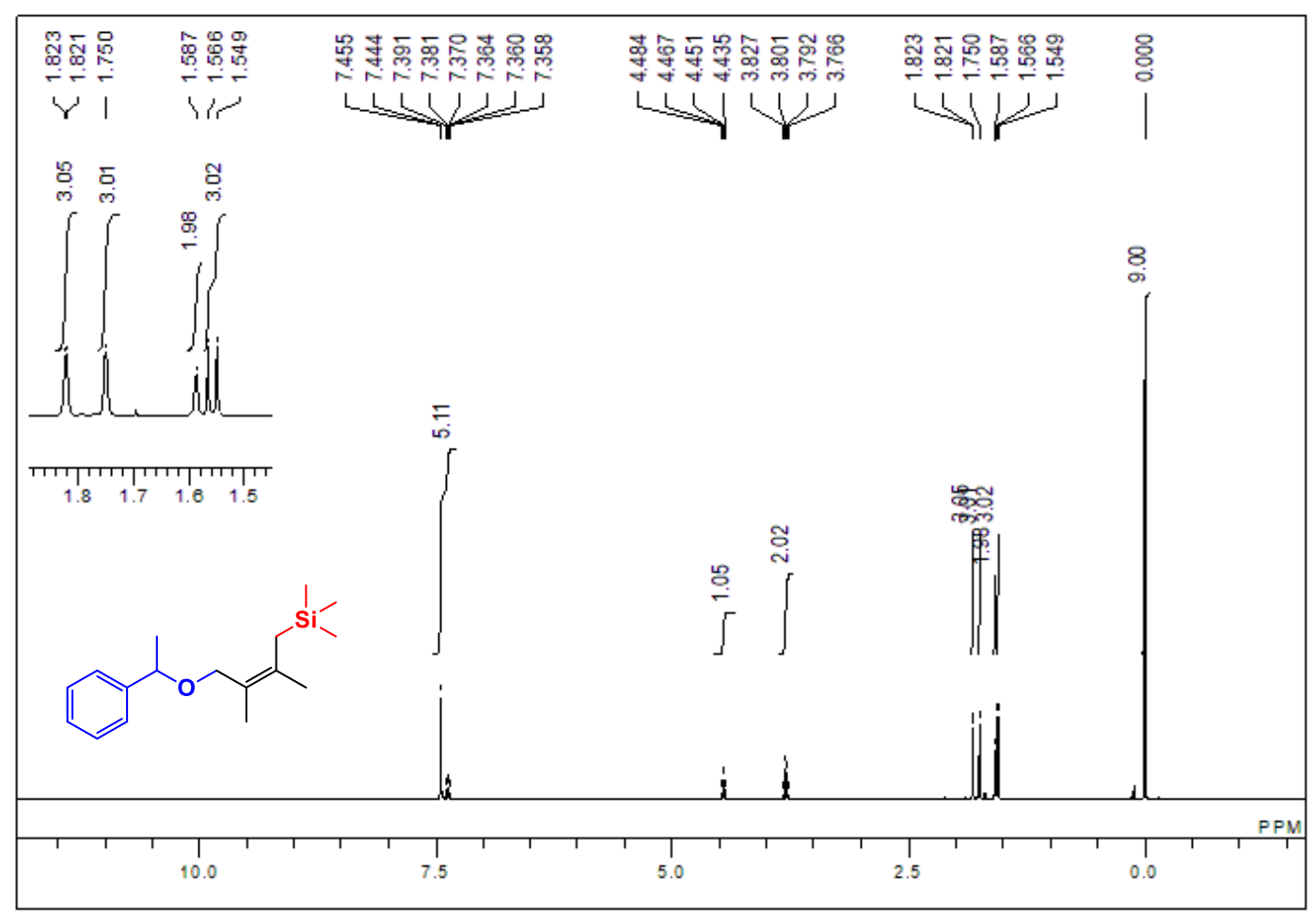

4i ${ }^{13} \mathrm{C} \mathrm{NMR}\left(100 \mathrm{MHz} ; \mathrm{CDCl}_{3}\right)$ :

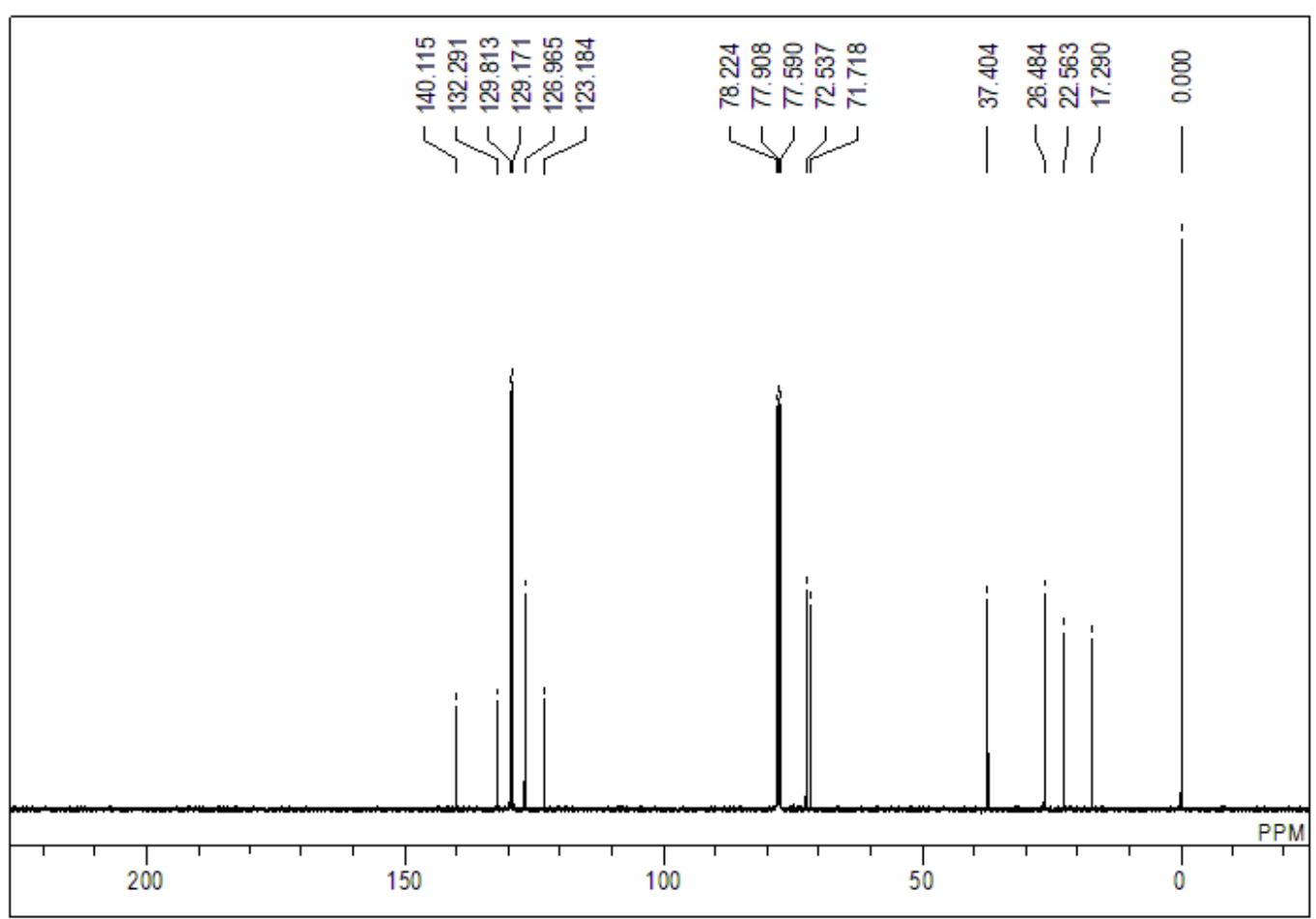


4i NOE (400 MHz; $\left.\mathrm{CDCl}_{3}\right)$ :
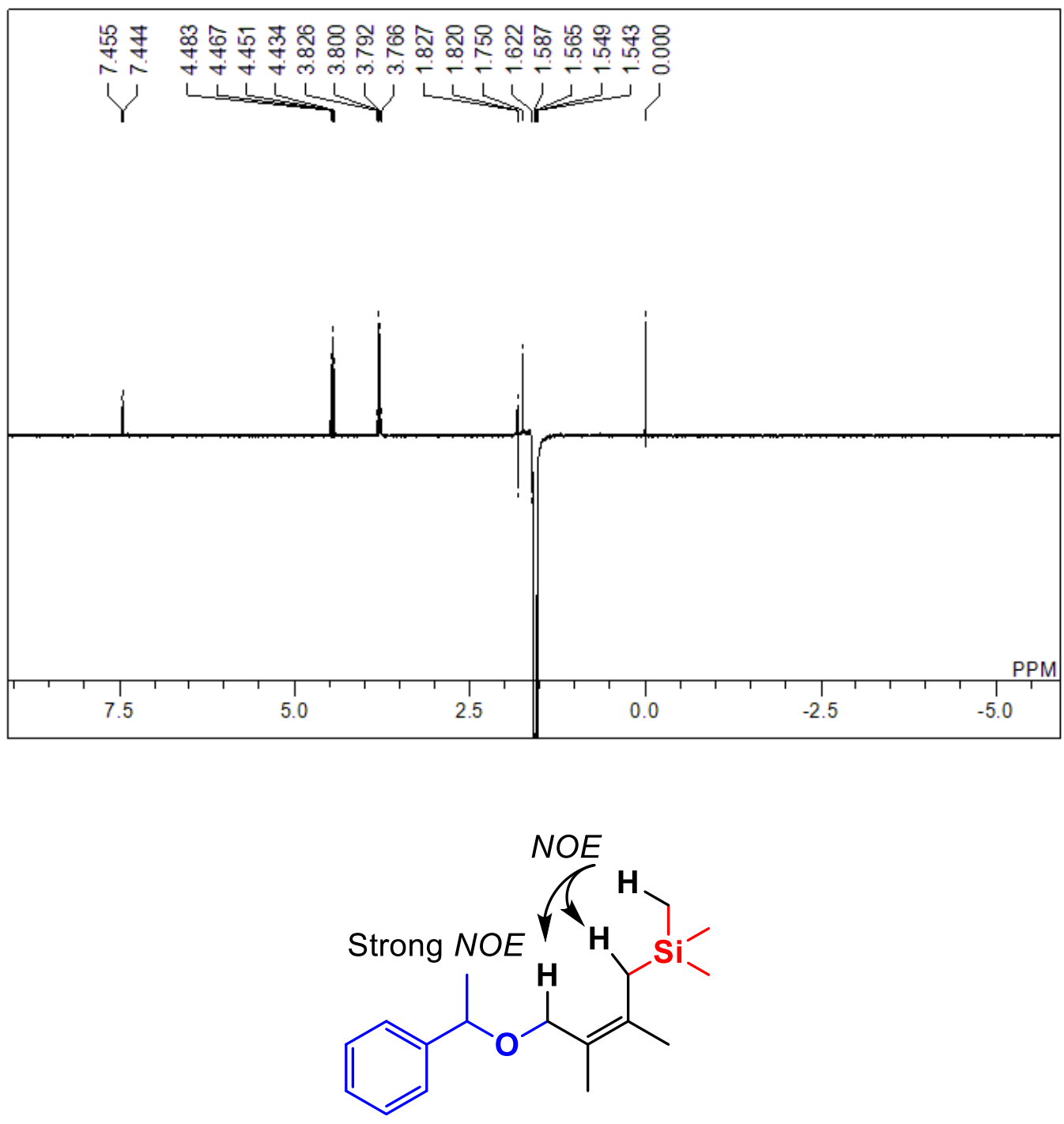

4i: Irradiation at the methylene proton-bearing TMS group $(\delta=1.59)$ caused strong enhancement of the methylene proton $(\delta=3.80)$. 
4j ${ }^{1} \mathrm{H}$ NMR (400 MHz; $\mathrm{CDCl}_{3}$ ):

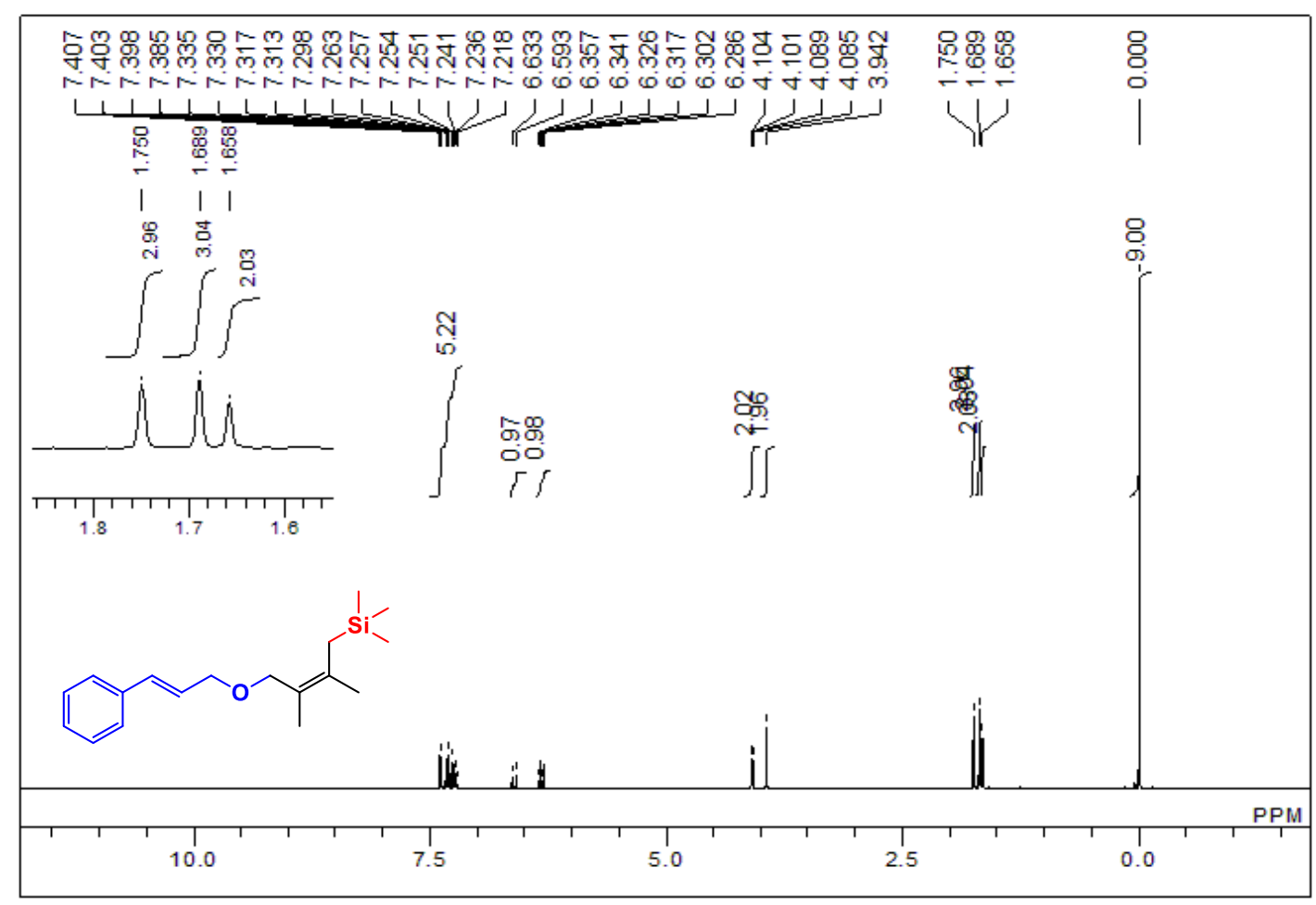

$4 \mathbf{j}{ }^{13} \mathrm{C}$ NMR (100 MHz; $\left.\mathrm{CDCl}_{3}\right)$ :

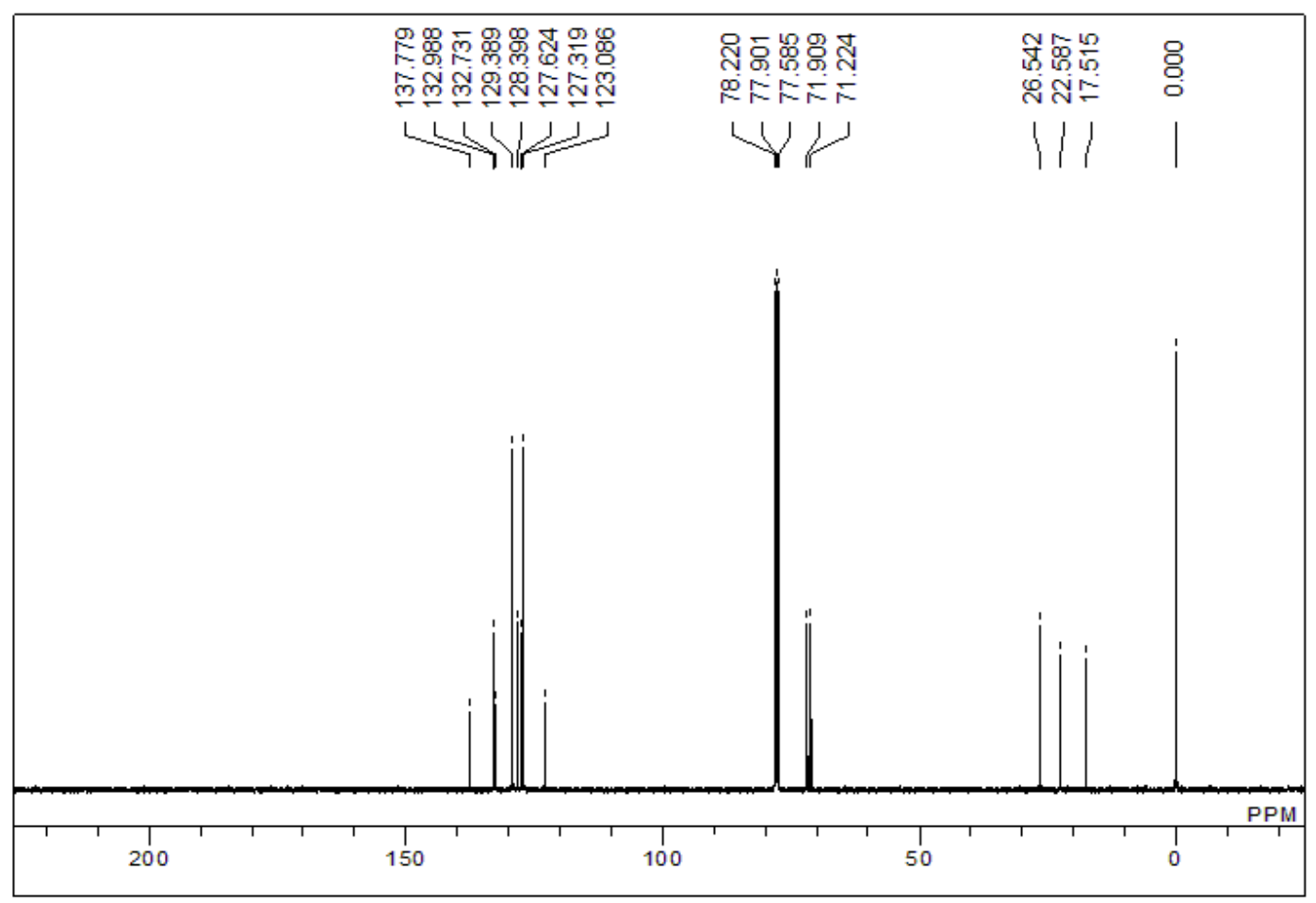


4j $\mathrm{NOE}\left(400 \mathrm{MHz} ; \mathrm{CDCl}_{3}\right)$ :
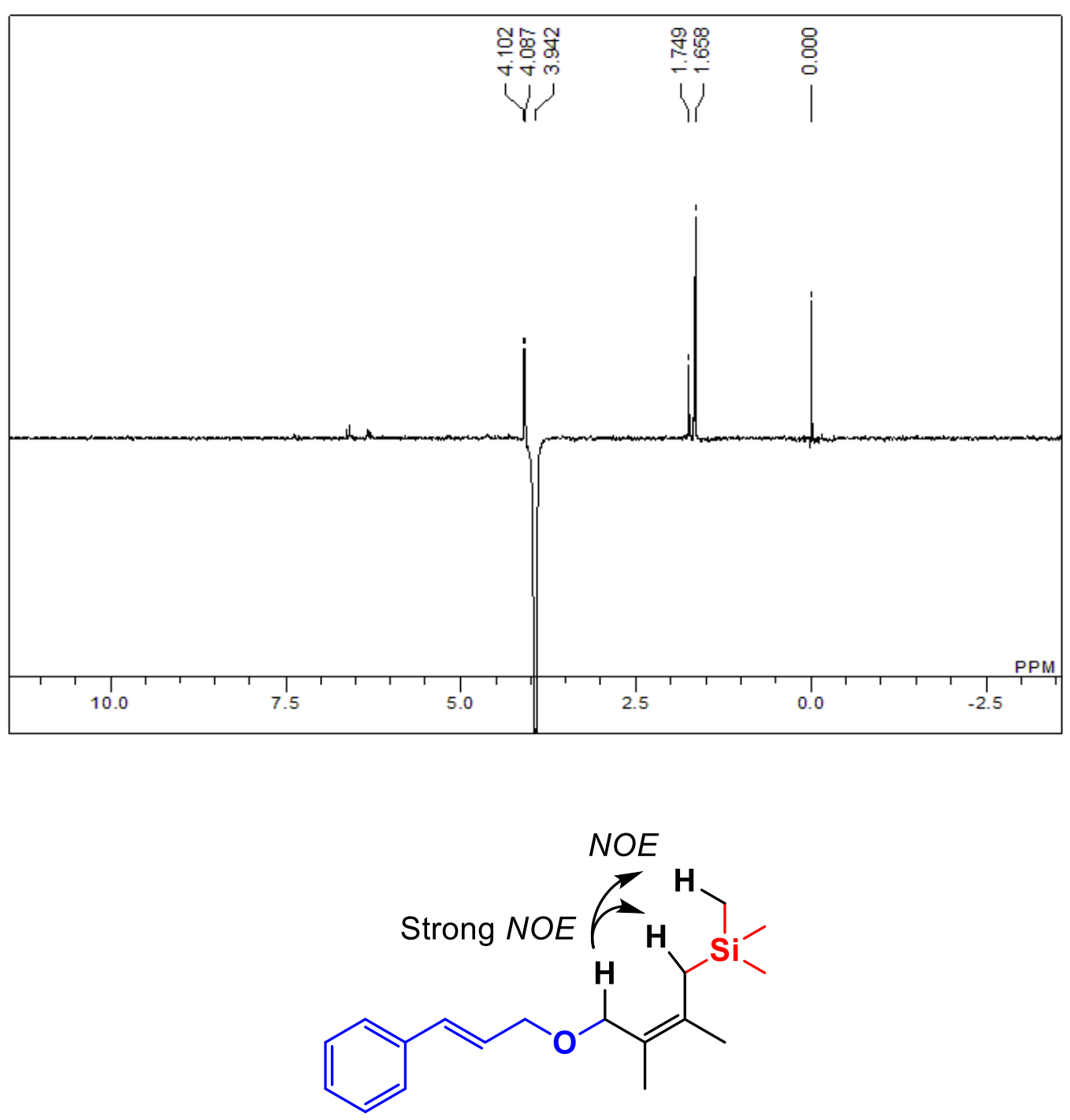

$4 \mathbf{j}$ : Irradiation at the methylene proton-bearing ether group $(\delta=3.94)$ caused strong enhancement of the methylene proton $(\delta=1.66)$. 
4k ${ }^{1} \mathrm{H}$ NMR (400 MHz; $\left.\mathrm{CDCl}_{3}\right)$ :

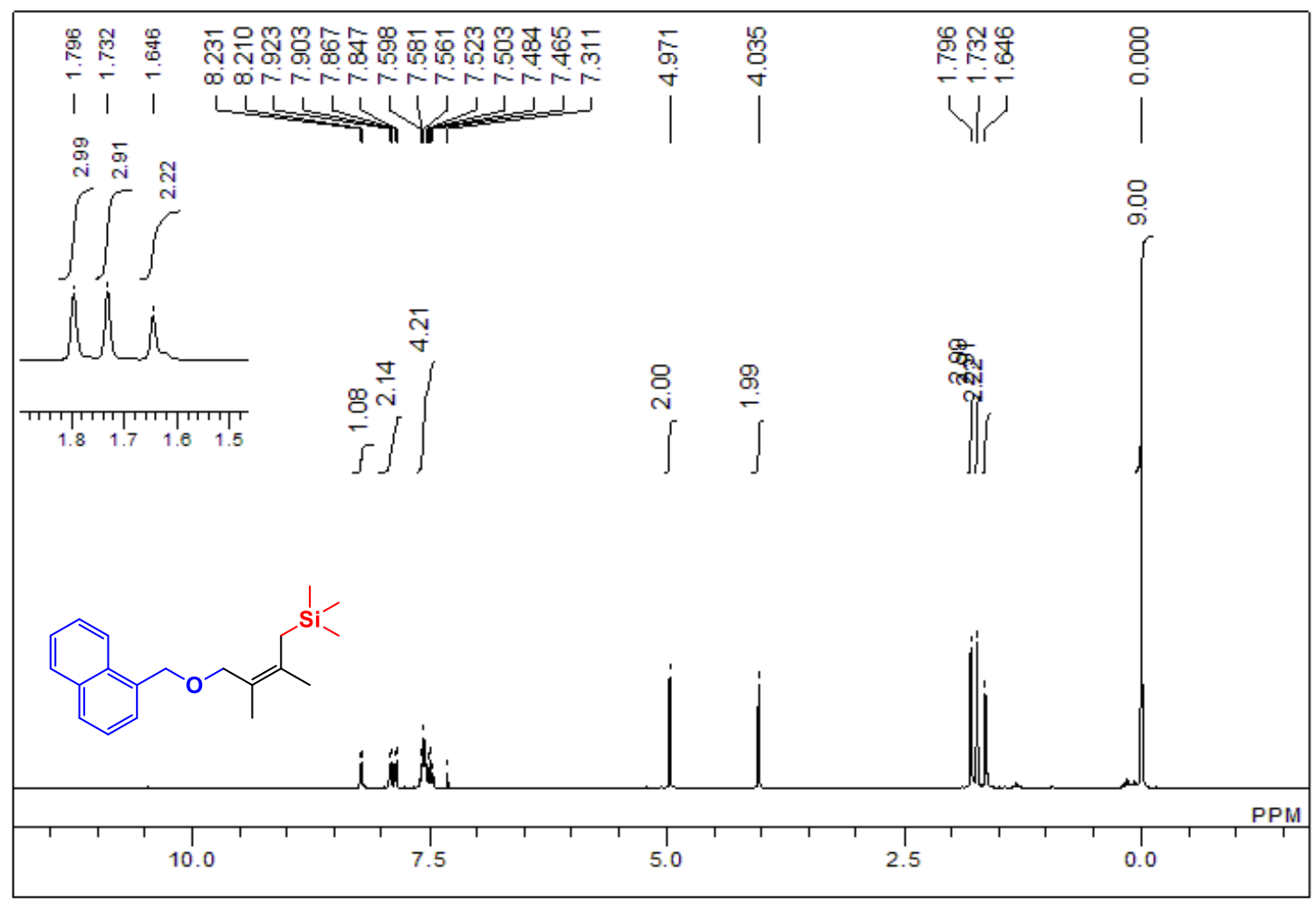

4k ${ }^{13} \mathrm{C}$ NMR (100 MHz; $\left.\mathrm{CDCl}_{3}\right)$ :

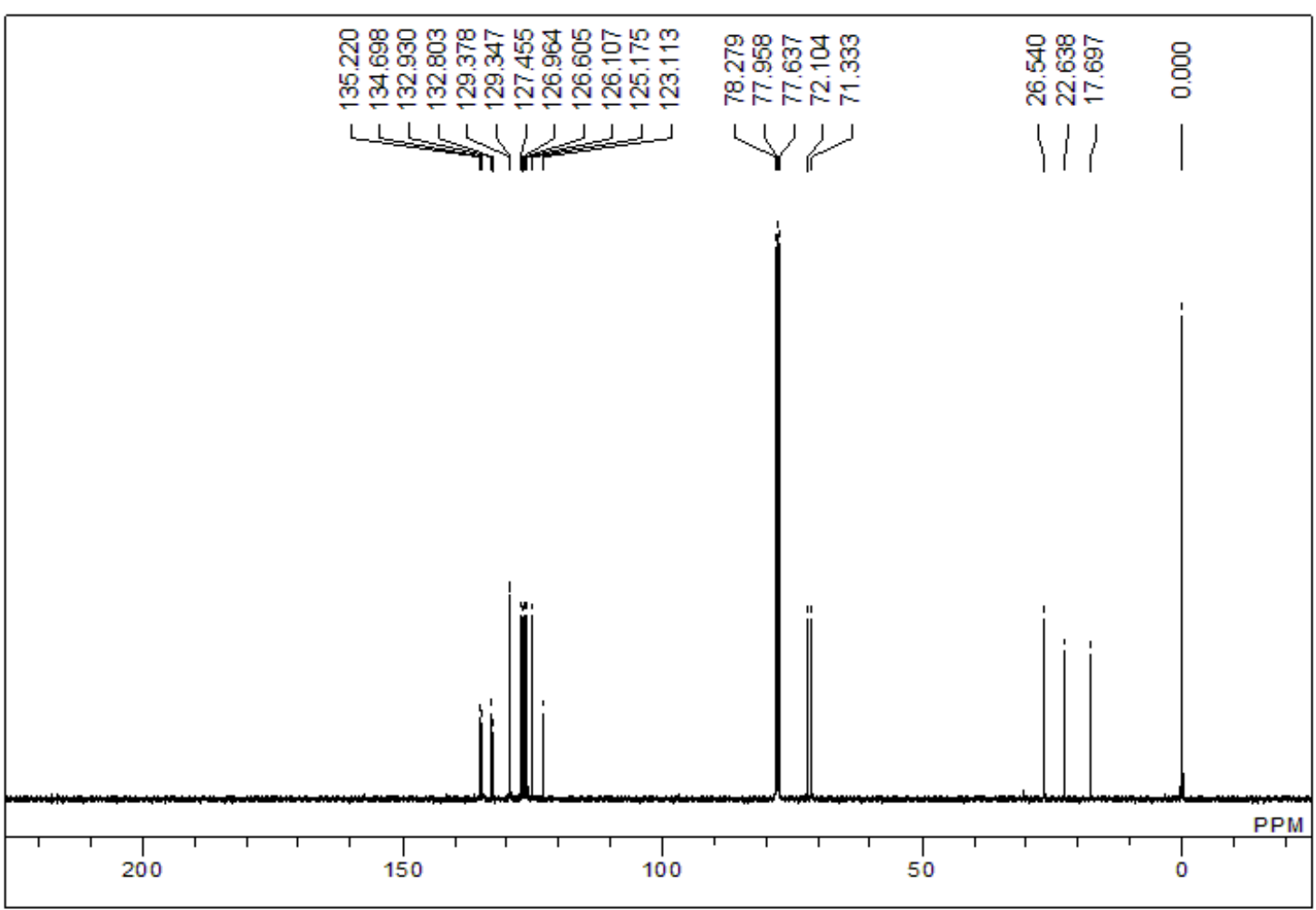


4k NOE (400 MHz; $\left.\mathrm{CDCl}_{3}\right)$ :

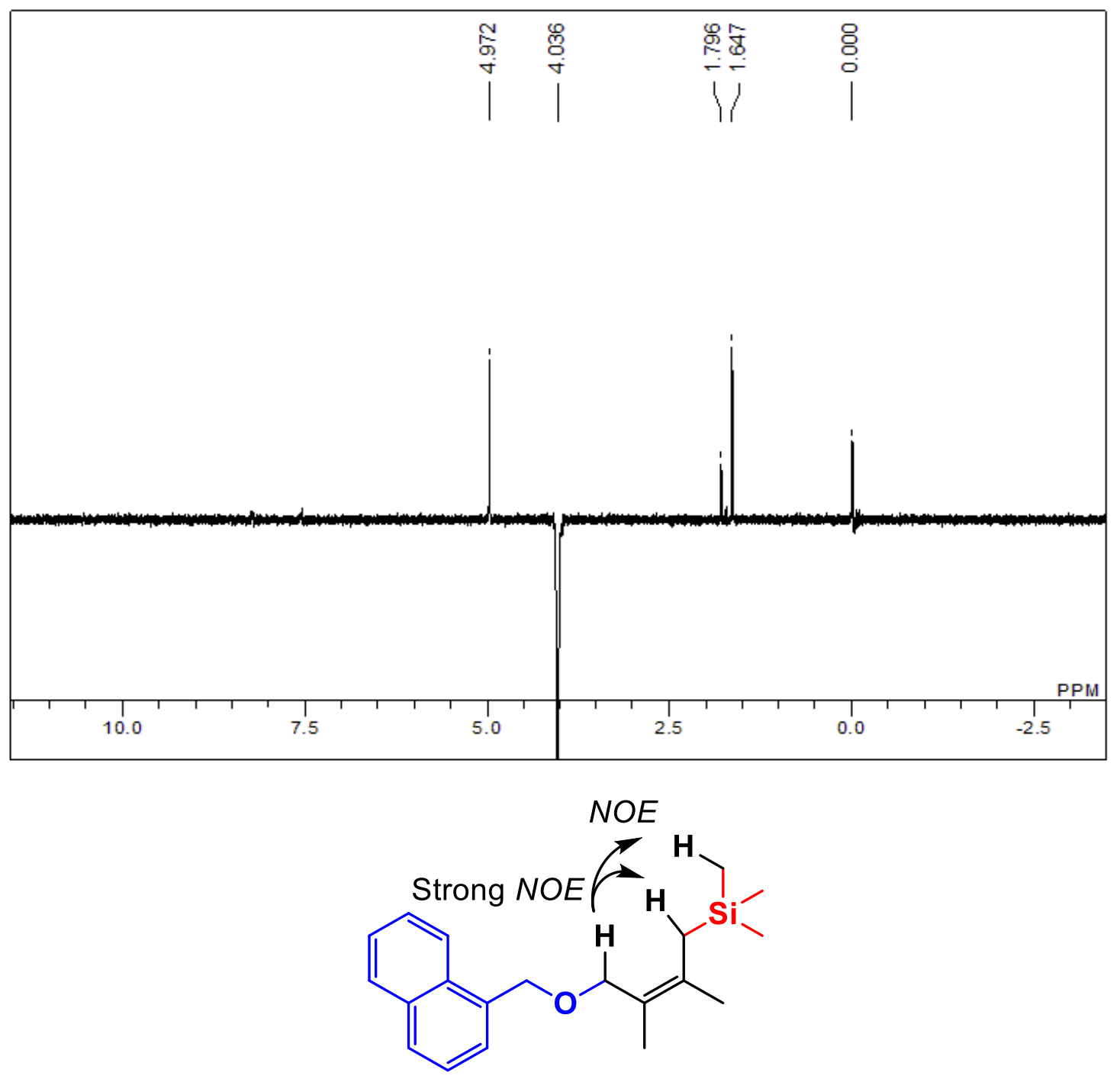

4k: Irradiation at the methylene proton-bearing ether group $(\delta=4.04)$ caused strong enhancement of the methylene proton $(\delta=1.65)$. 
$4 \mathbf{l}^{1} \mathrm{H}$ NMR (400 MHz; $\left.\mathrm{CDCl}_{3}\right)$ :

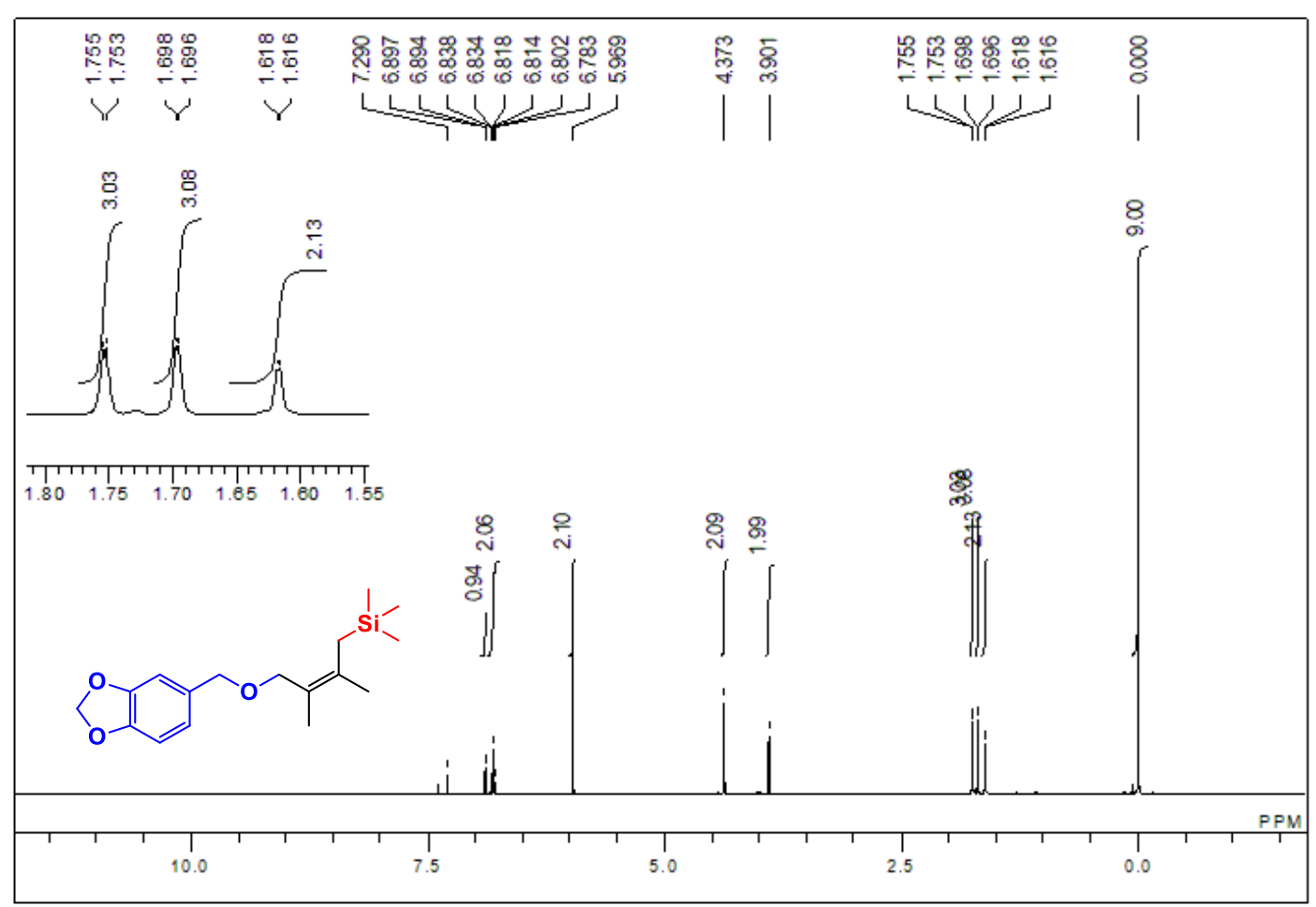

$4{ }^{13} \mathrm{C}$ NMR (100 MHz; $\left.\mathrm{CDCl}_{3}\right)$ :

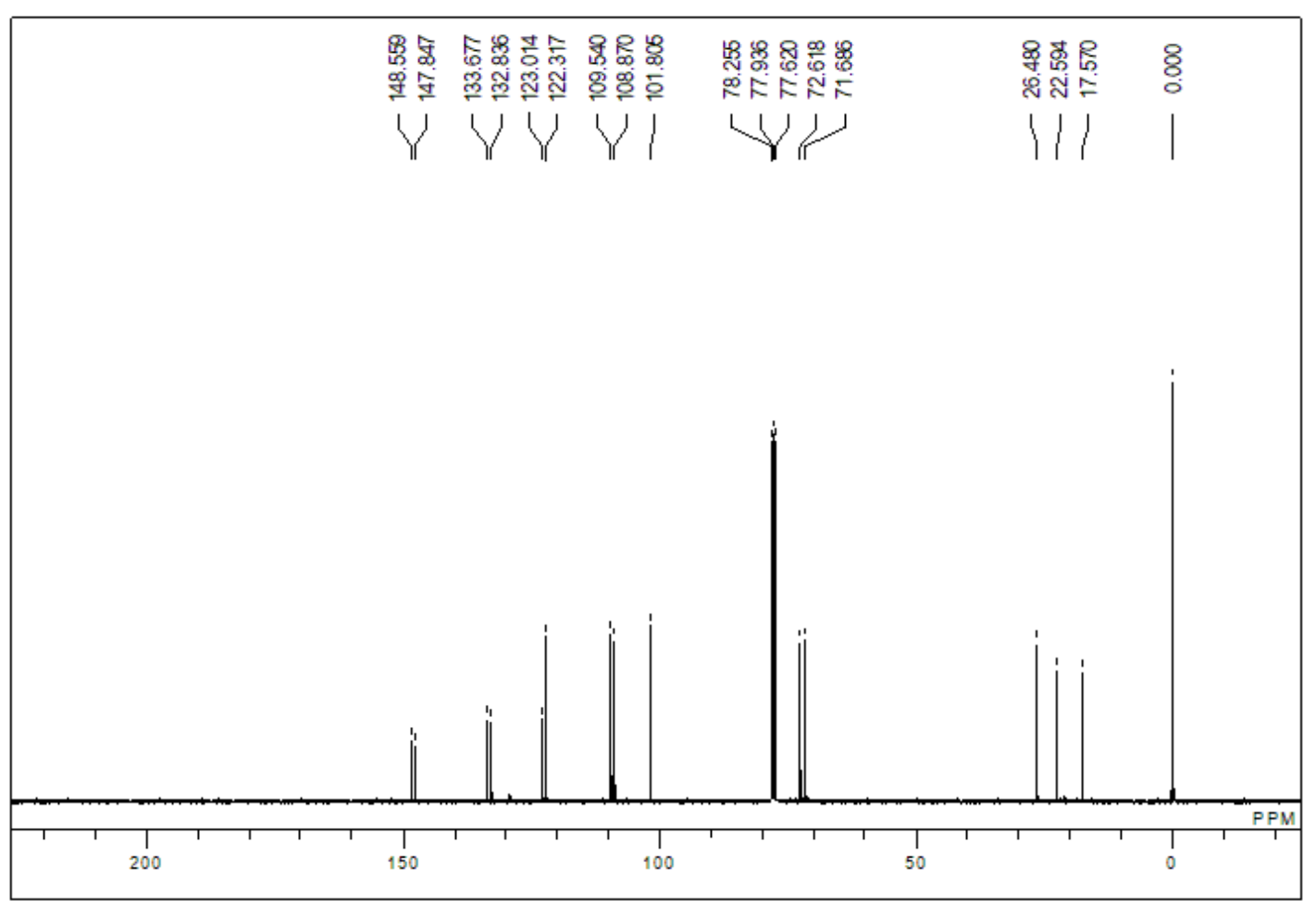


$41 \mathrm{NOE}\left(400 \mathrm{MHz} ; \mathrm{CDCl}_{3}\right)$ :
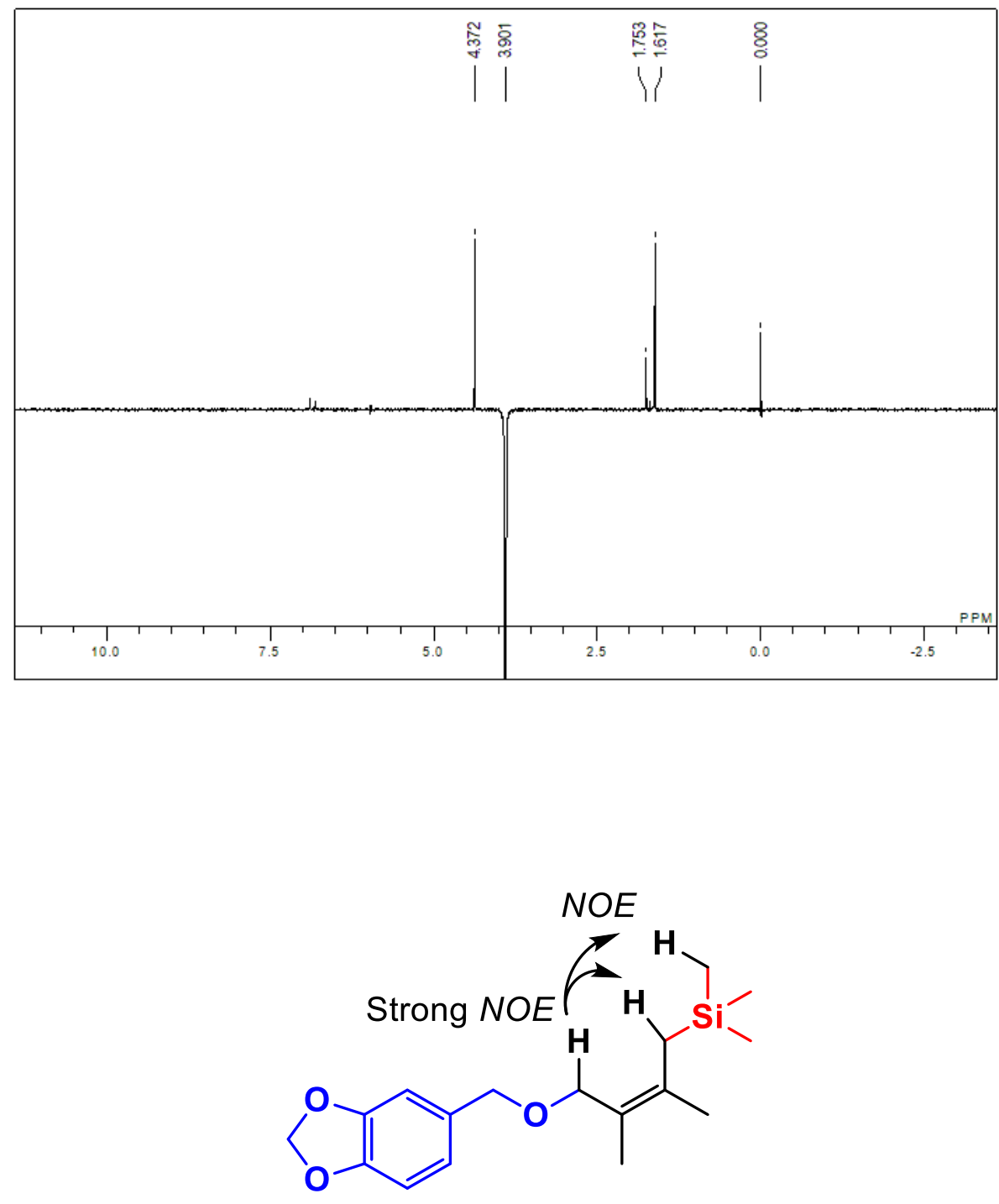

4I: Irradiation at the methylene proton-bearing ether group $(\delta=3.90)$ caused strong enhancement of the methylene proton $(\delta=1.62)$. 
4m ${ }^{1} \mathrm{H}$ NMR (400 MHz; $\mathrm{CDCl}_{3}$ ):

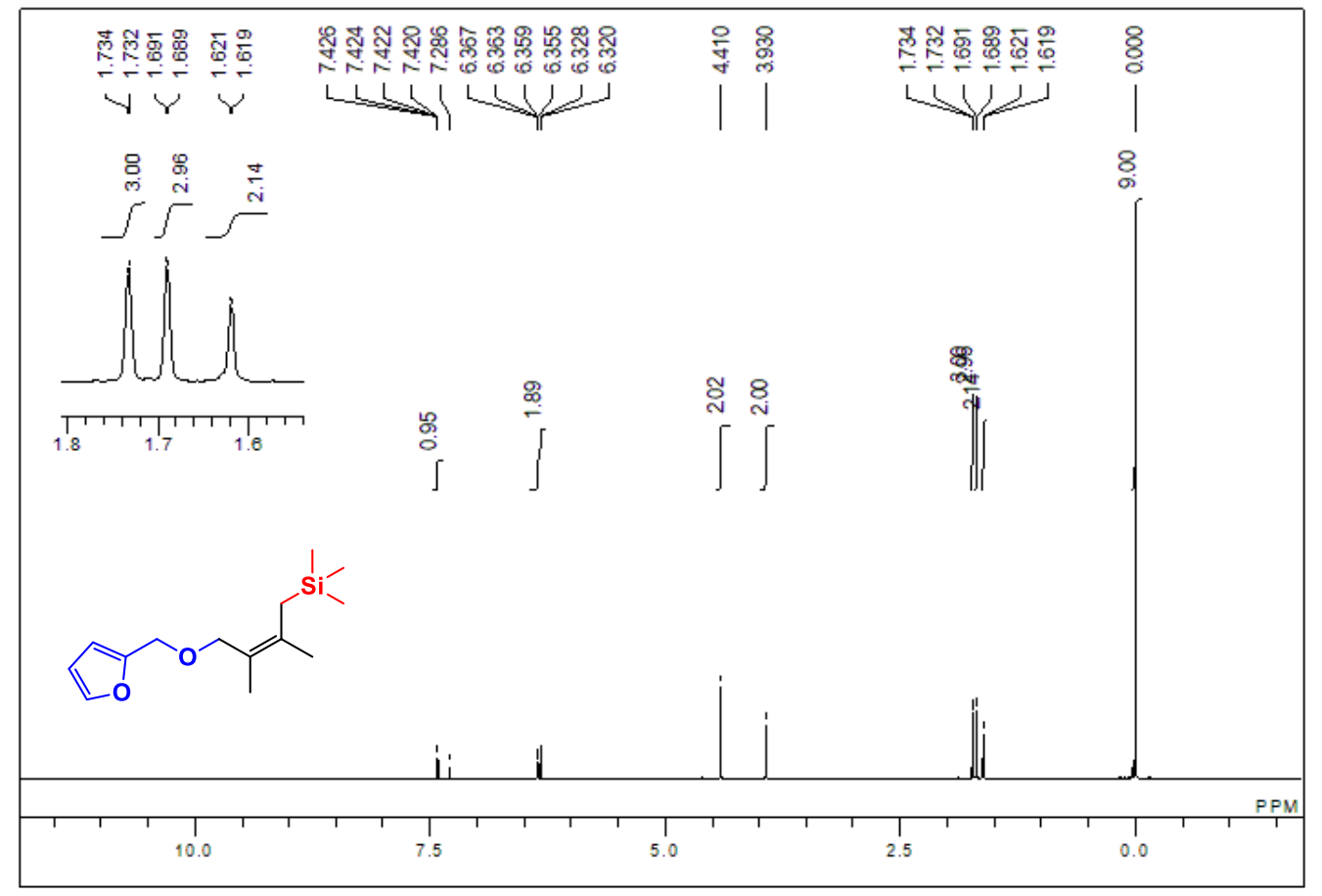

4m ${ }^{13} \mathrm{C}$ NMR (100 MHz; $\left.\mathrm{CDCl}_{3}\right)$ :

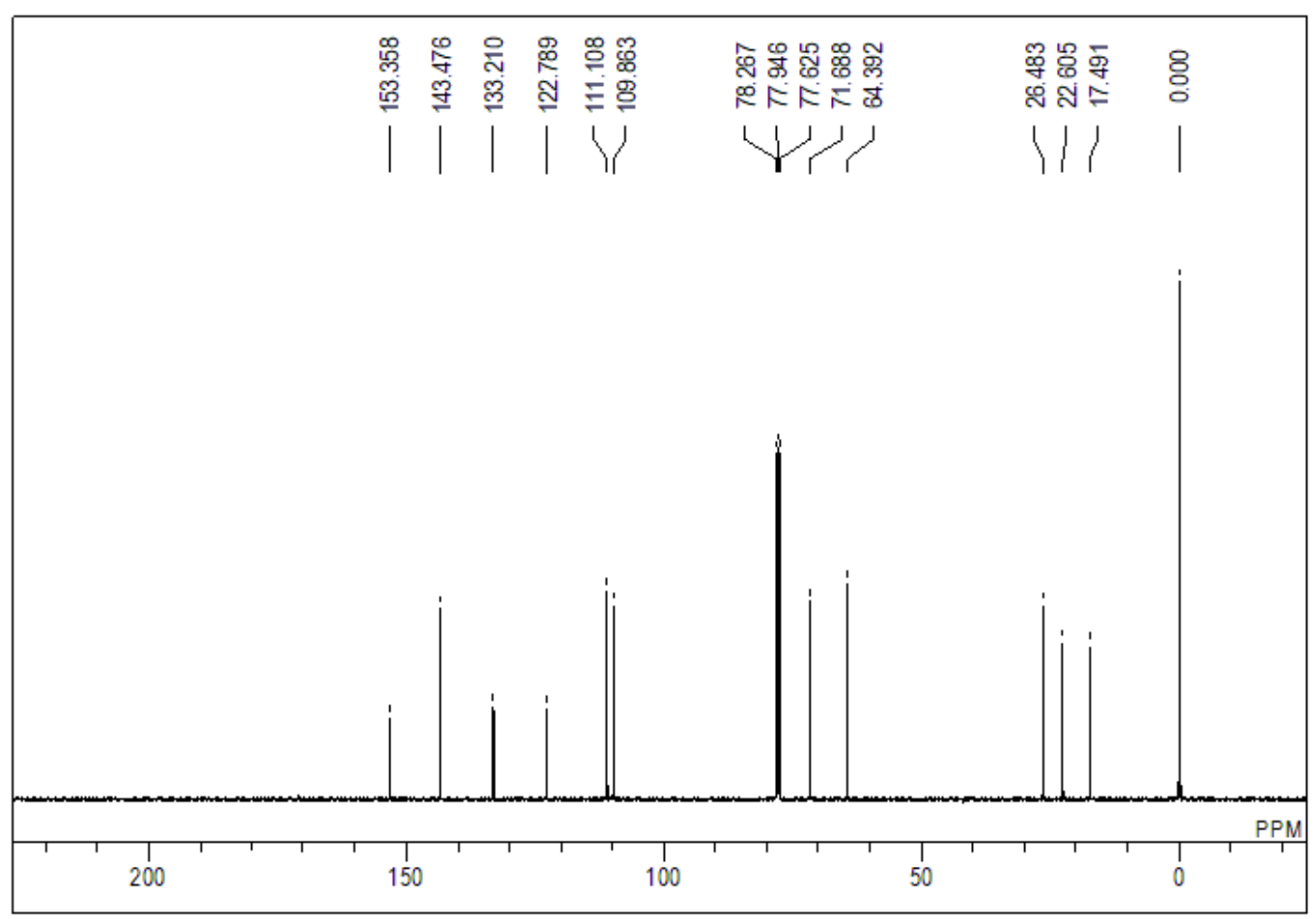


4m NOE (400 MHz; $\left.\mathrm{CDCl}_{3}\right)$ :
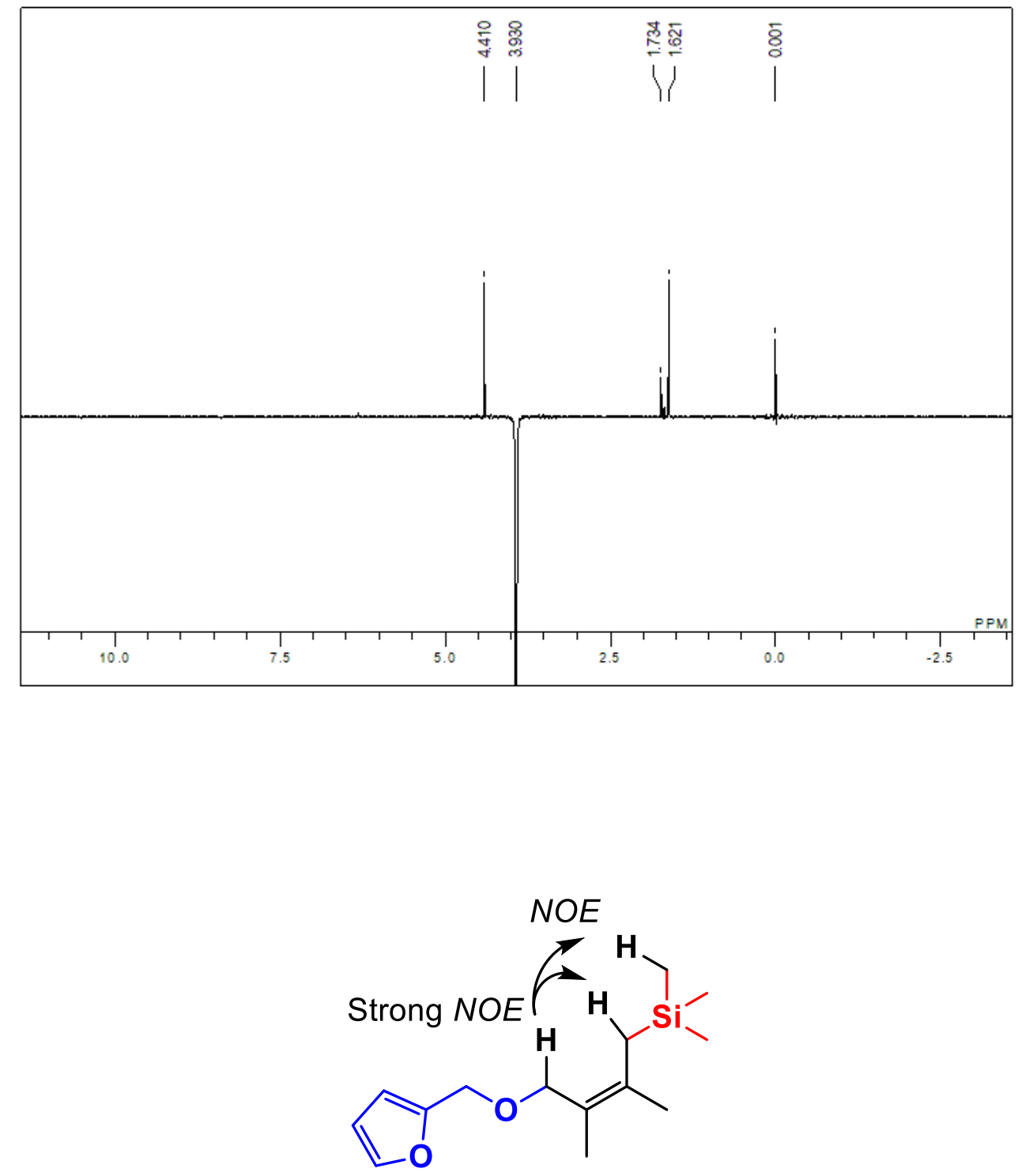

$\mathbf{4 m}$ : Irradiation at the methylene proton-bearing ether group $(\delta=3.93)$ caused strong enhancement of the methylene proton $(\delta=1.62)$. 
4n ${ }^{1} \mathrm{H}$ NMR (400 MHz; $\left.\mathrm{CDCl}_{3}\right)$ :

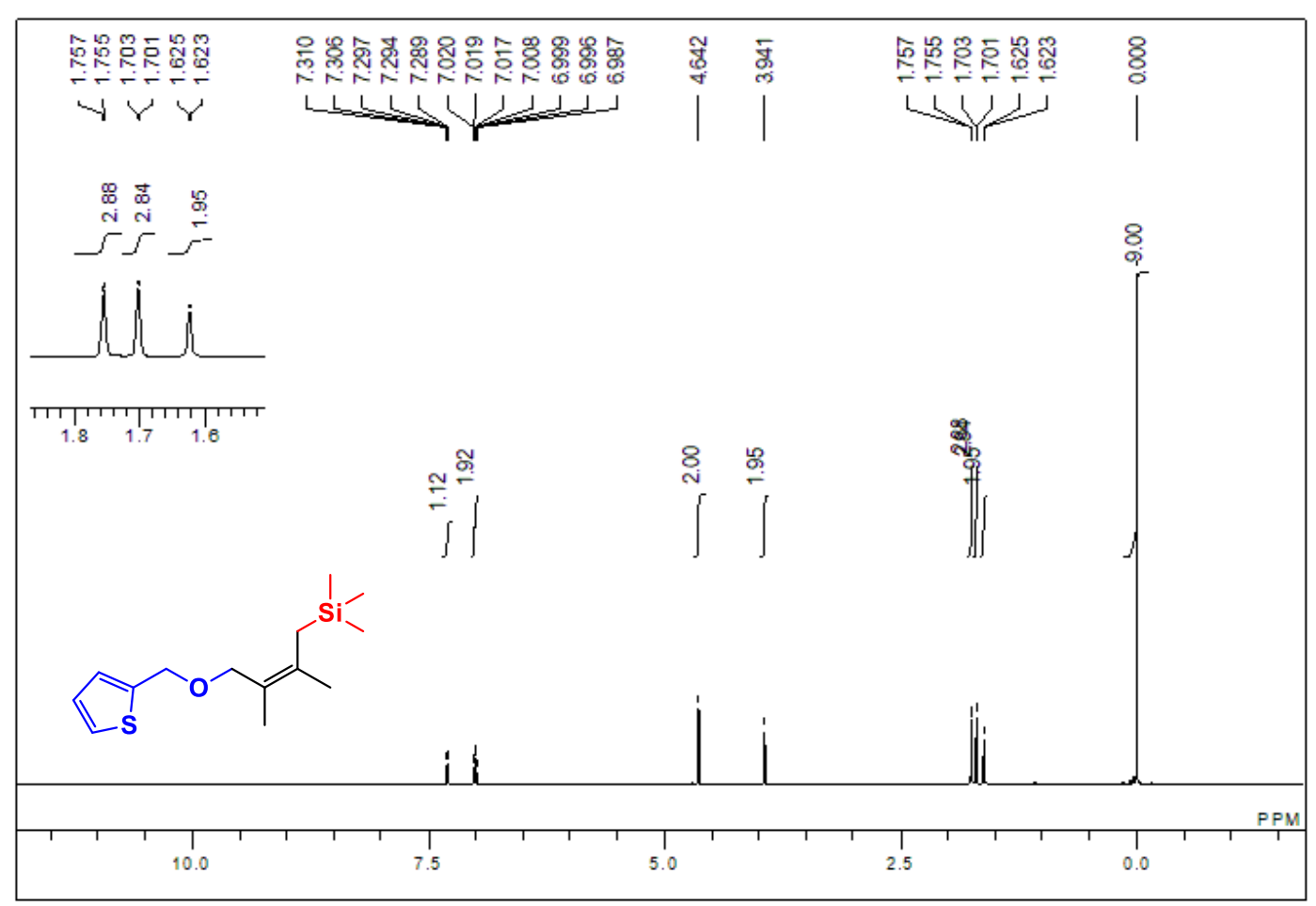

4n ${ }^{13} \mathrm{C}$ NMR (100 MHz; $\left.\mathrm{CDCl}_{3}\right)$ :

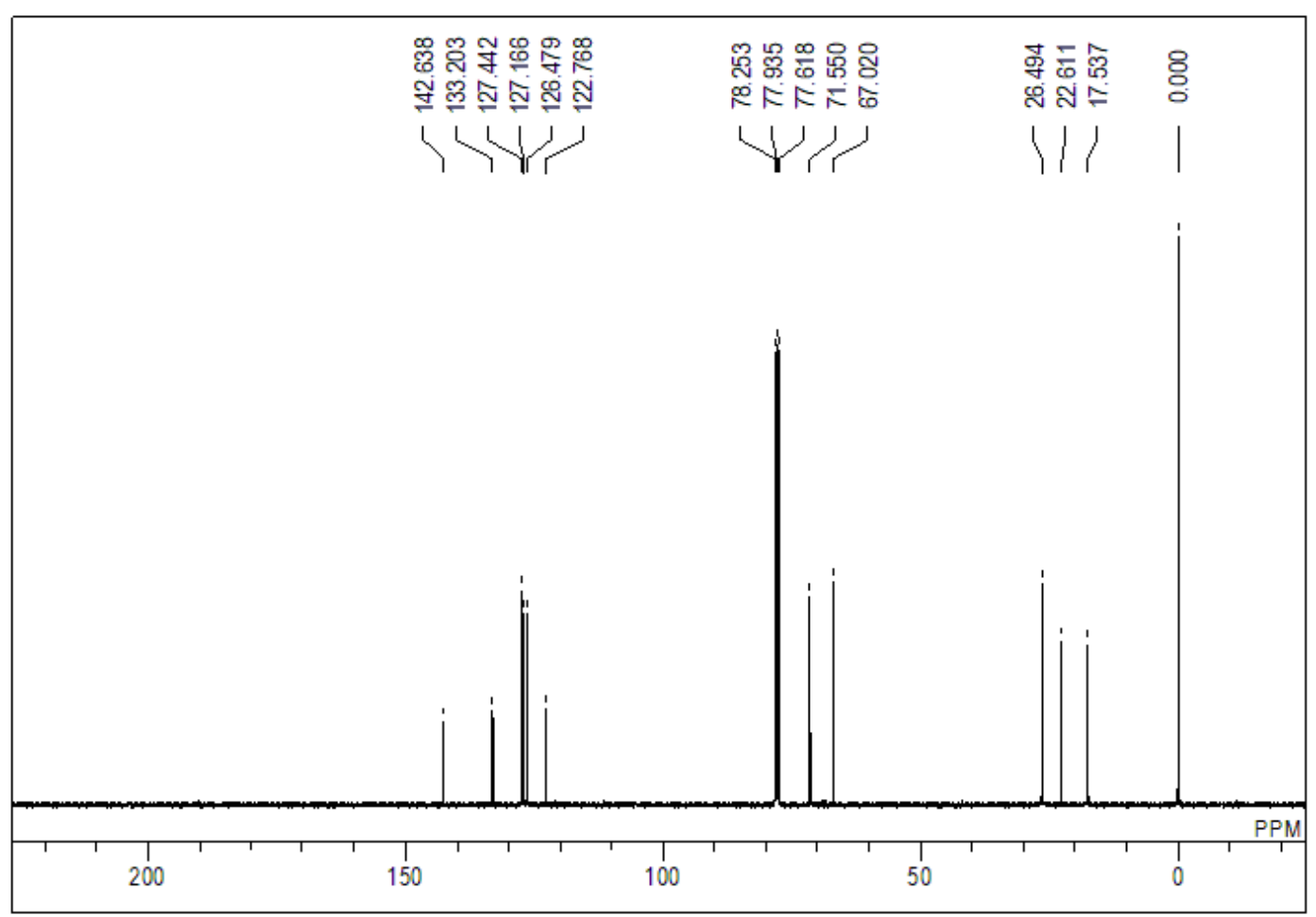


4n NOE (400 MHz; $\left.\mathrm{CDCl}_{3}\right)$ :
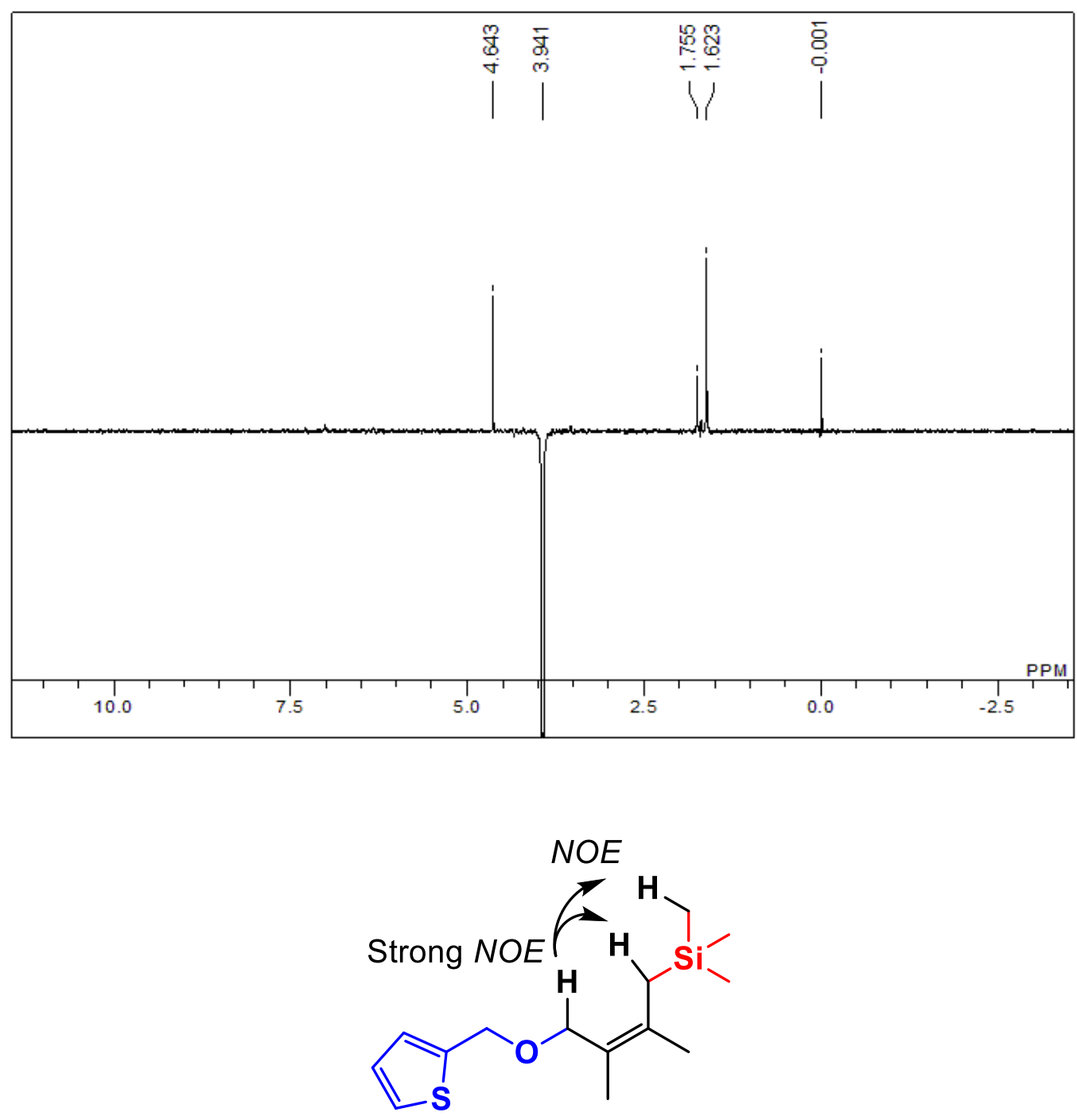

4n: Irradiation at the methylene proton-bearing ether group $(\delta=3.94)$ caused strong enhancement of the methylene proton $(\delta=1.62)$. 
4o ${ }^{1} \mathrm{H}$ NMR (400 MHz; $\left.\mathrm{CDCl}_{3}\right)$ :

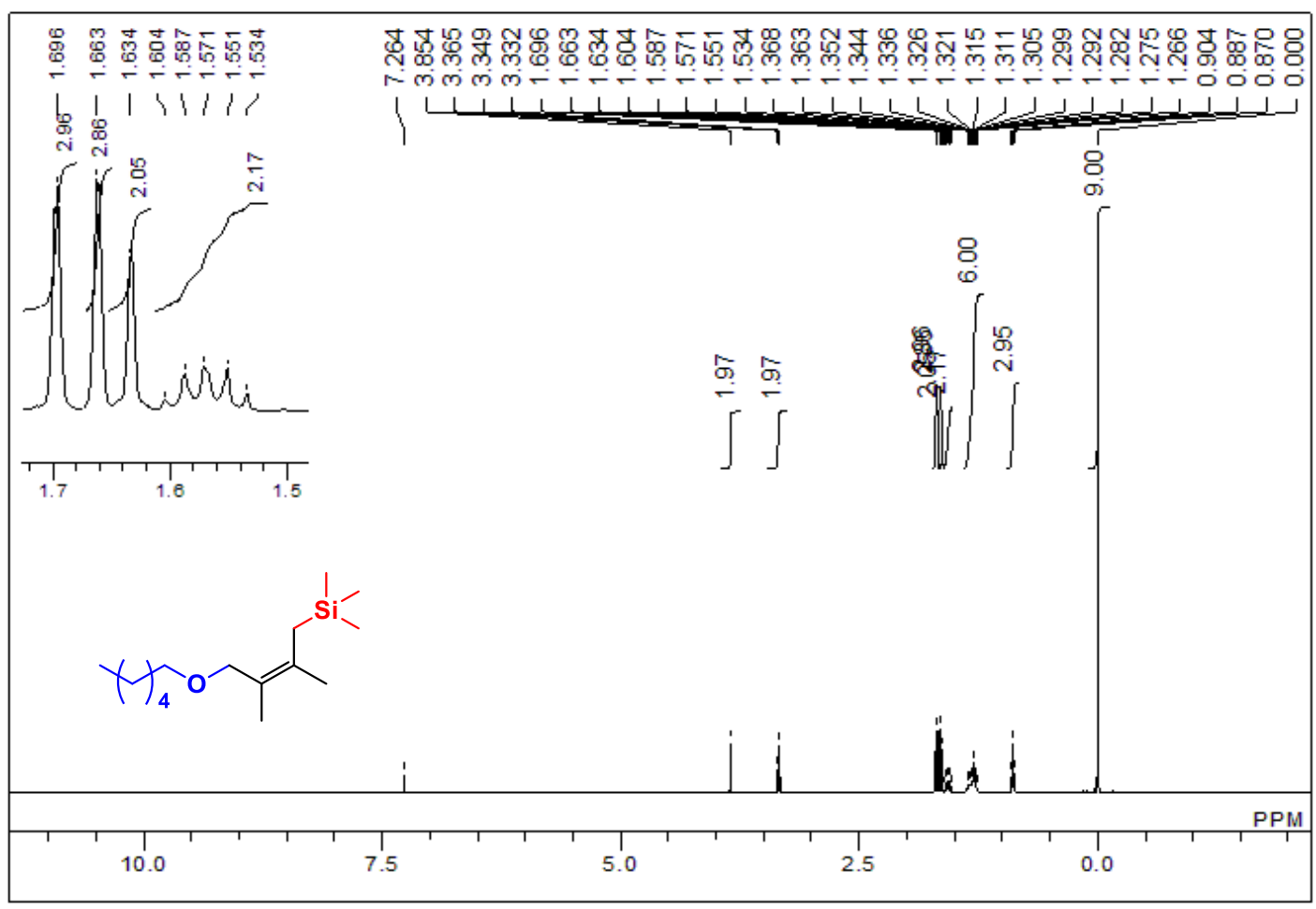

4o ${ }^{13} \mathrm{C}$ NMR (100 MHz; $\left.\mathrm{CDCl}_{3}\right)$ :

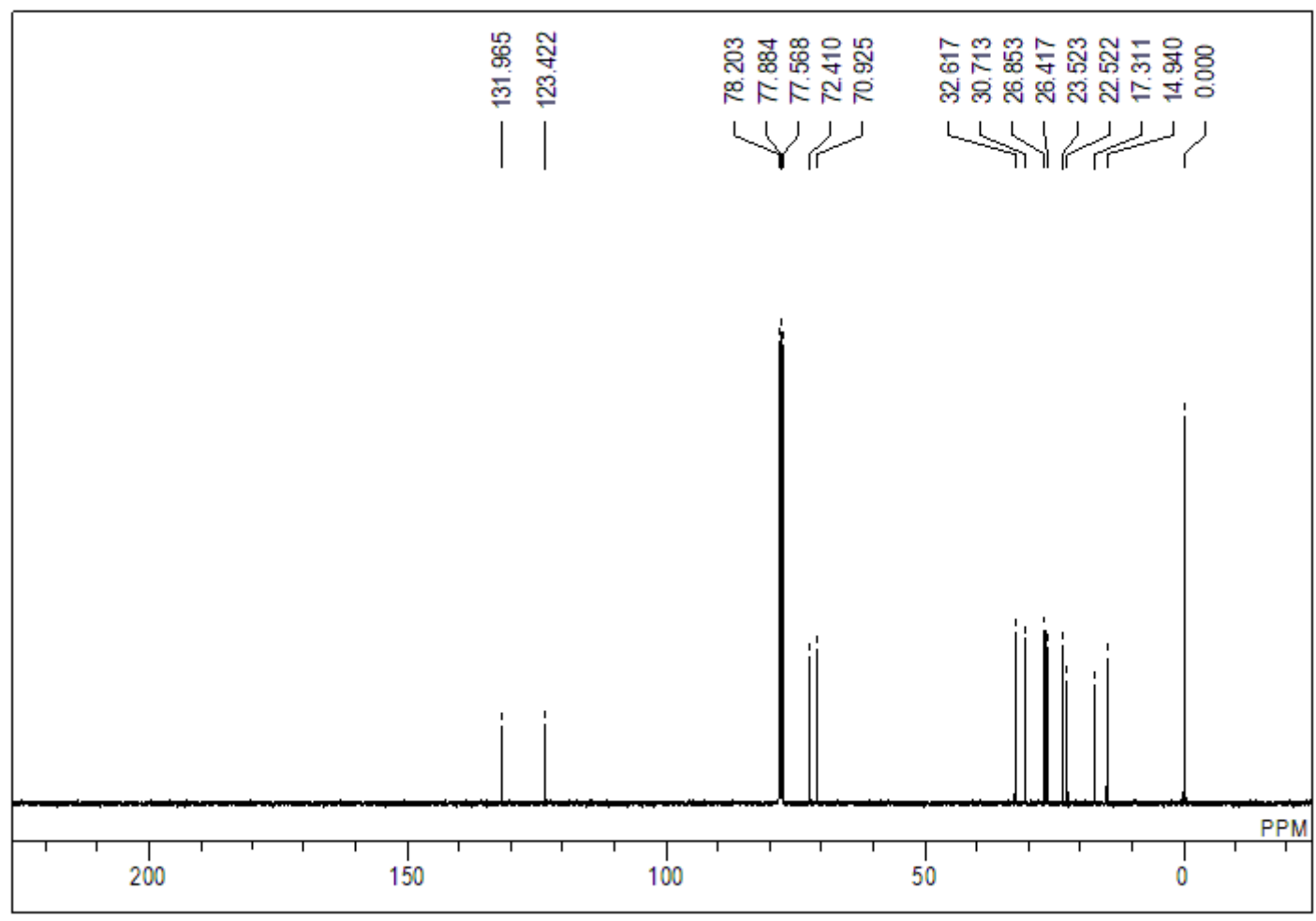


$40 \mathrm{NOE}\left(400 \mathrm{MHz} ; \mathrm{CDCl}_{3}\right)$ :
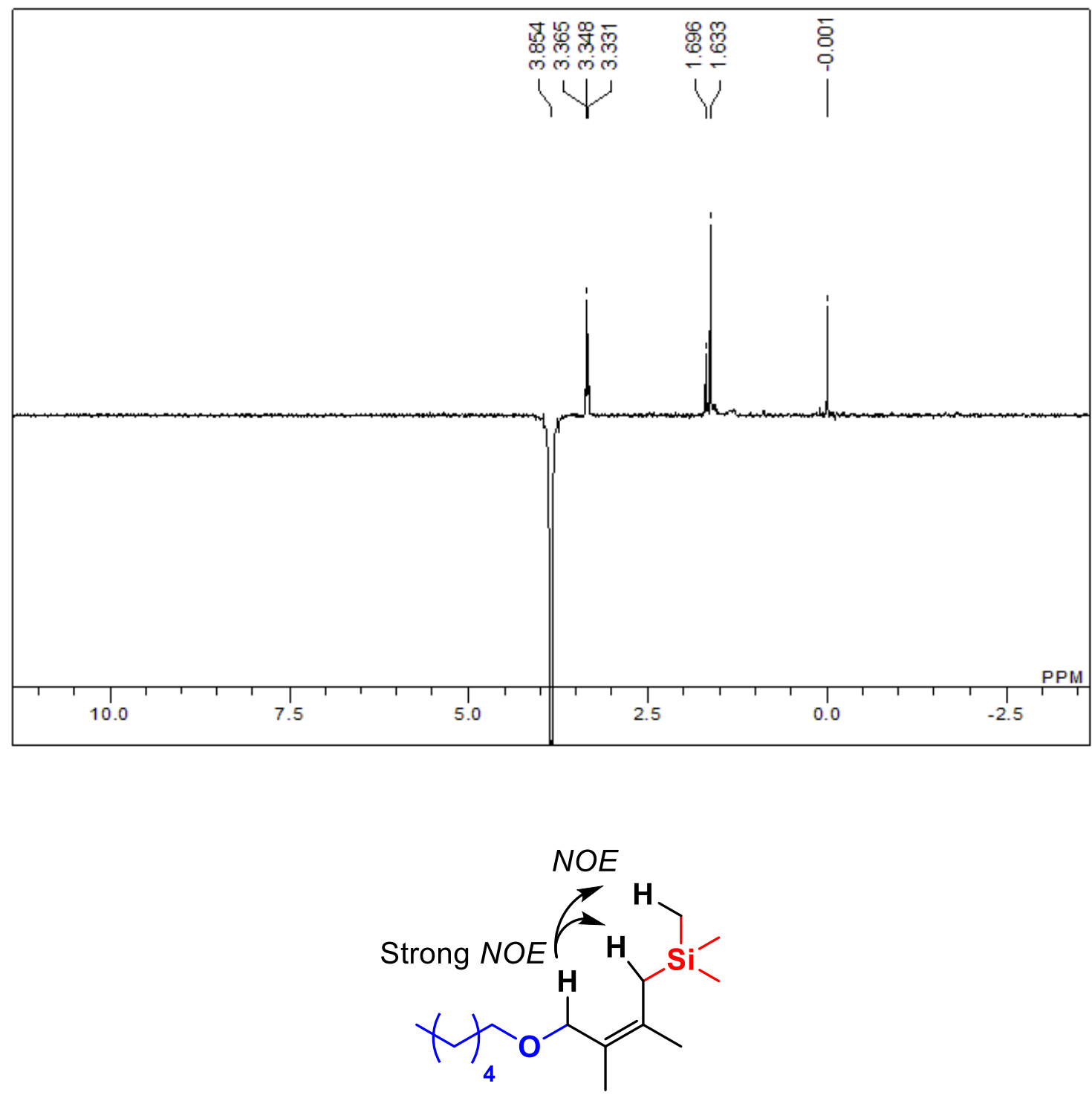

4o: Irradiation at the methylene proton-bearing ether group $(\delta=3.85)$ caused strong enhancement of the methylene proton $(\delta=1.63)$. 
4p ${ }^{1} \mathrm{H}$ NMR (400 MHz; $\left.\mathrm{CDCl}_{3}\right)$ :

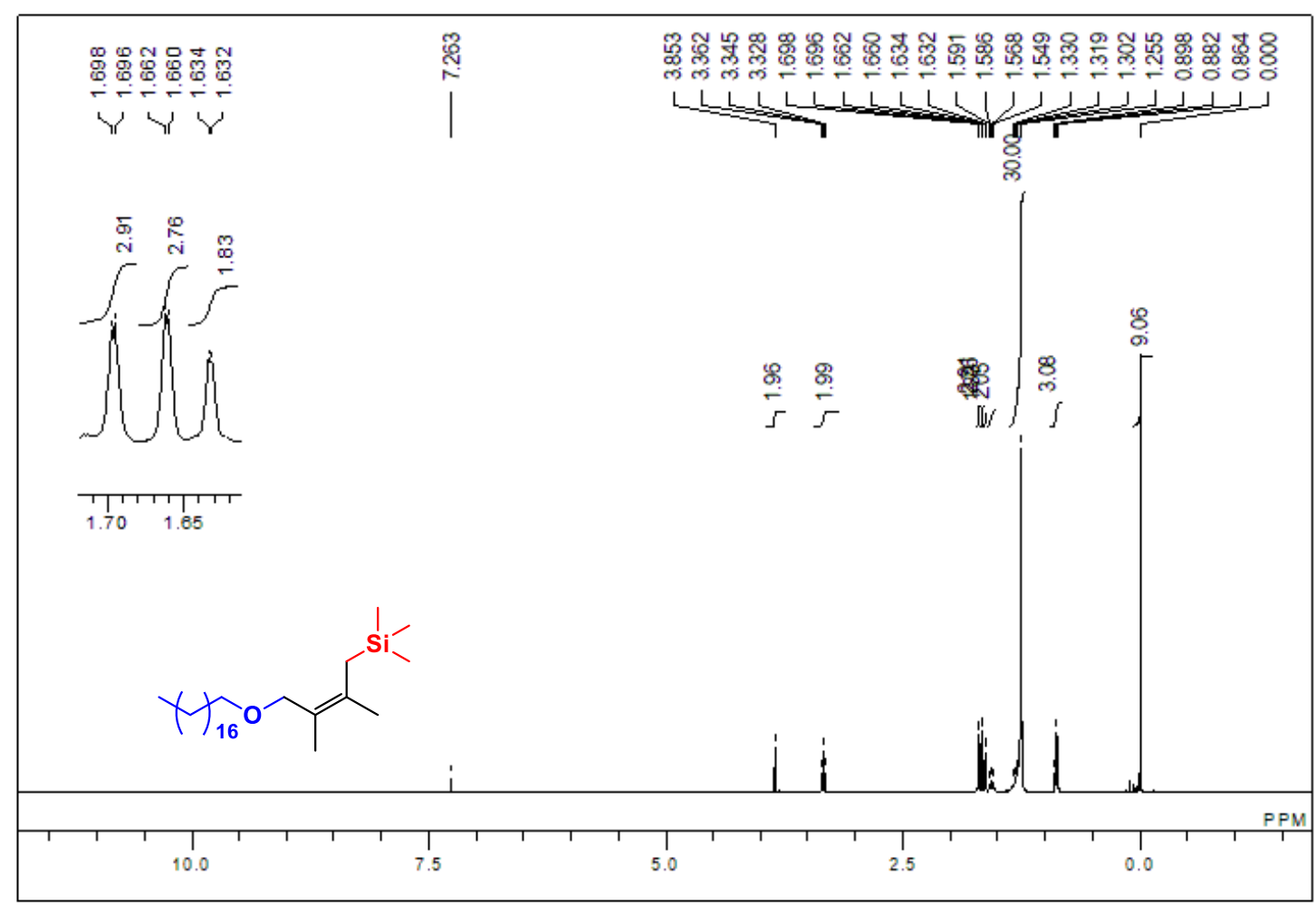

4p ${ }^{13} \mathrm{C}$ NMR (100 MHz; $\left.\mathrm{CDCl}_{3}\right)$ :

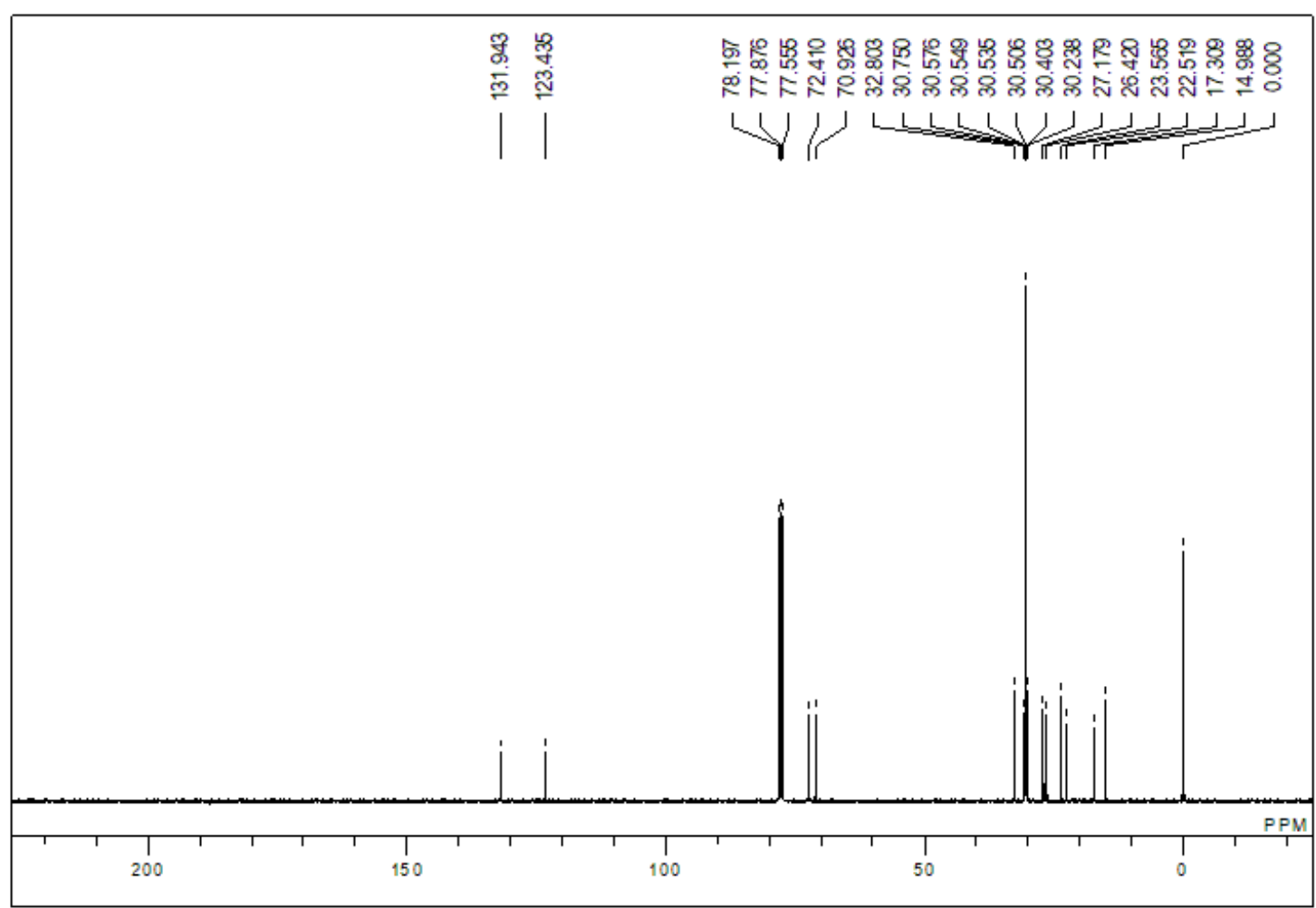


4p $\mathrm{NOE}\left(400 \mathrm{MHz} ; \mathrm{CDCl}_{3}\right)$ :
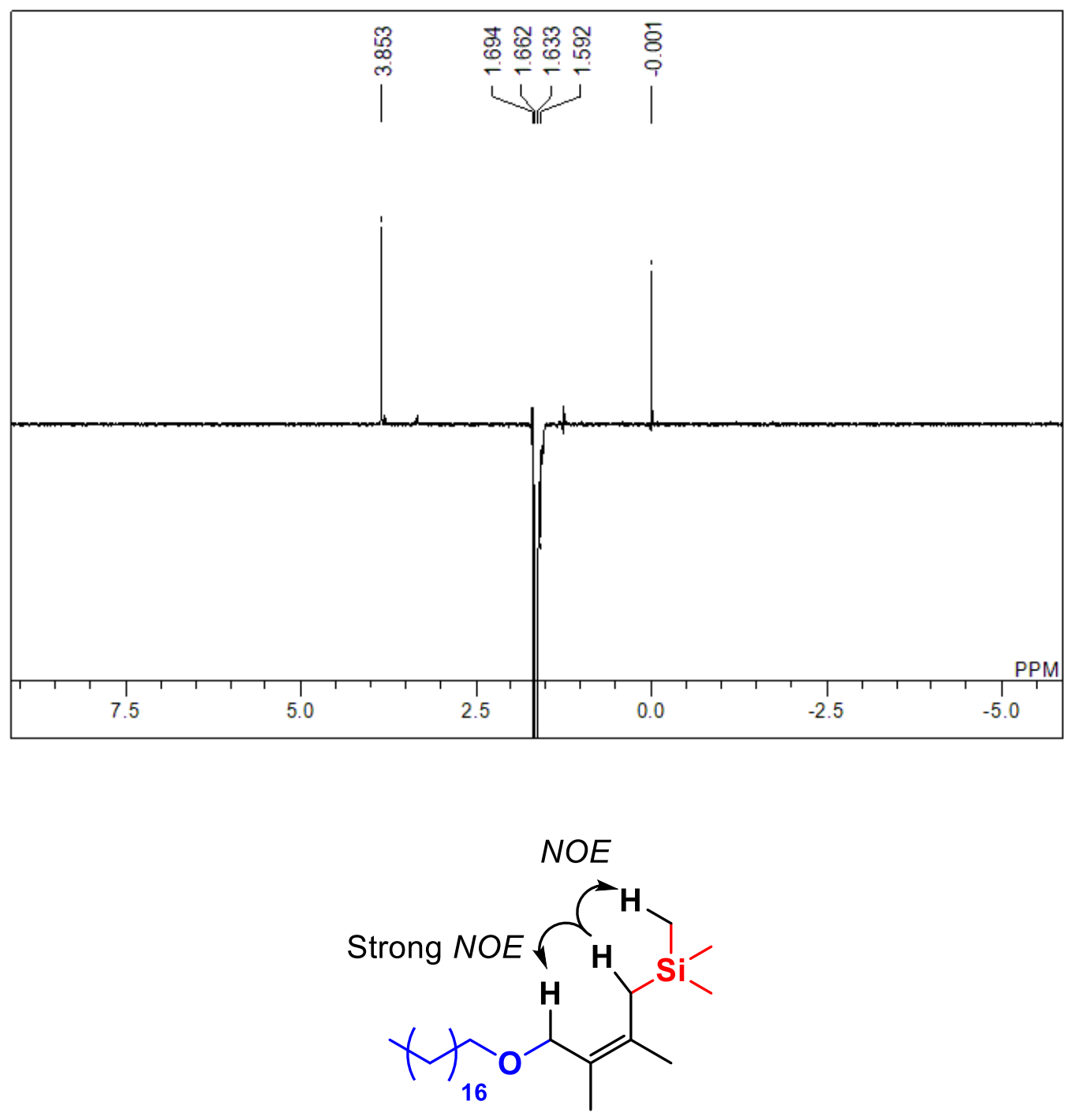

4p: Irradiation at the methylene proton-bearing TMS group $(\delta=1.63)$ caused strong enhancement of the methylene proton $(\delta=3.85)$. 
4q ${ }^{1} \mathrm{H}$ NMR (400 MHz; $\mathrm{CDCl}_{3}$ ):

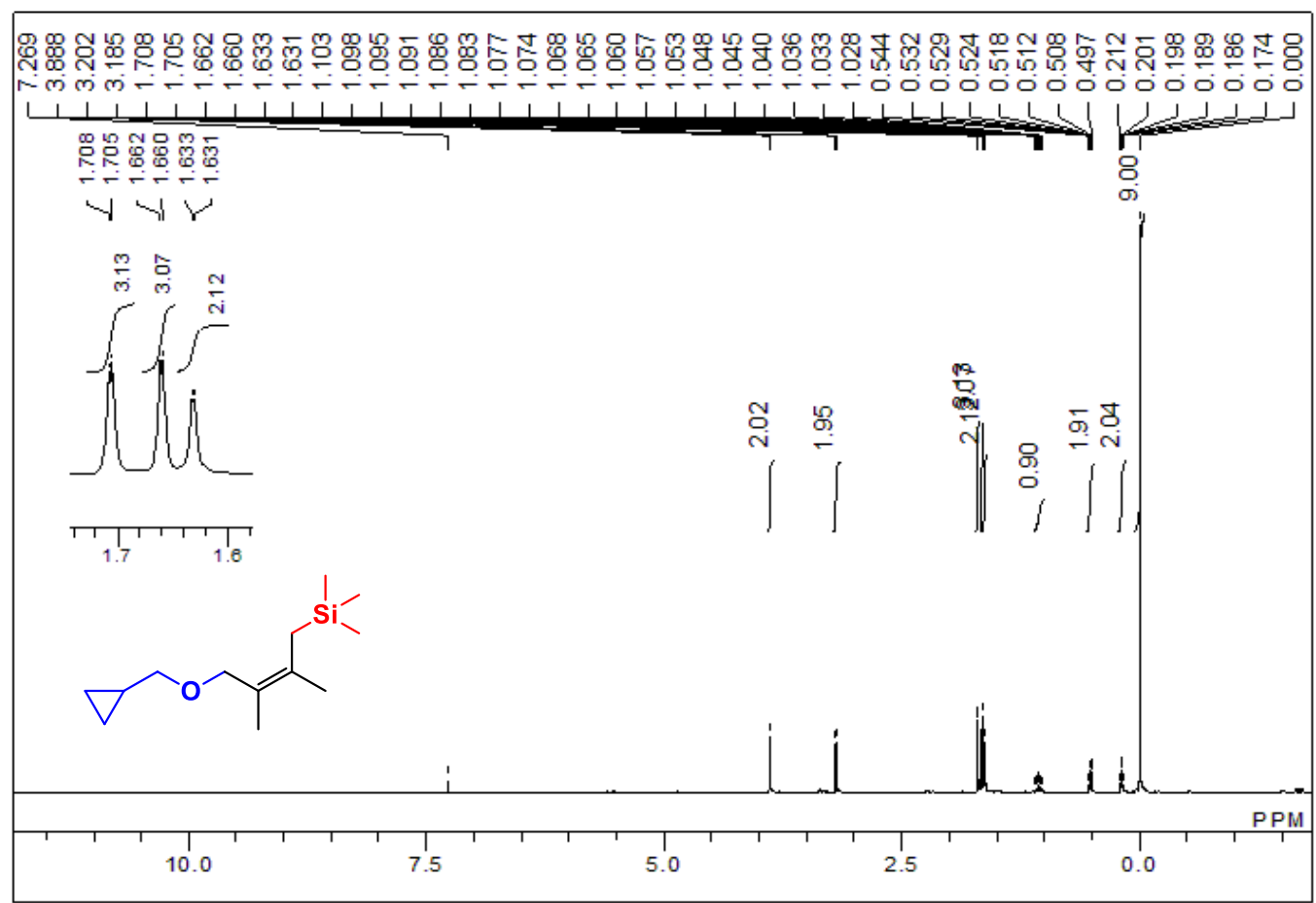

$4 \mathbf{q}{ }^{13} \mathrm{C}$ NMR (100 MHz; $\left.\mathrm{CDCl}_{3}\right)$ :

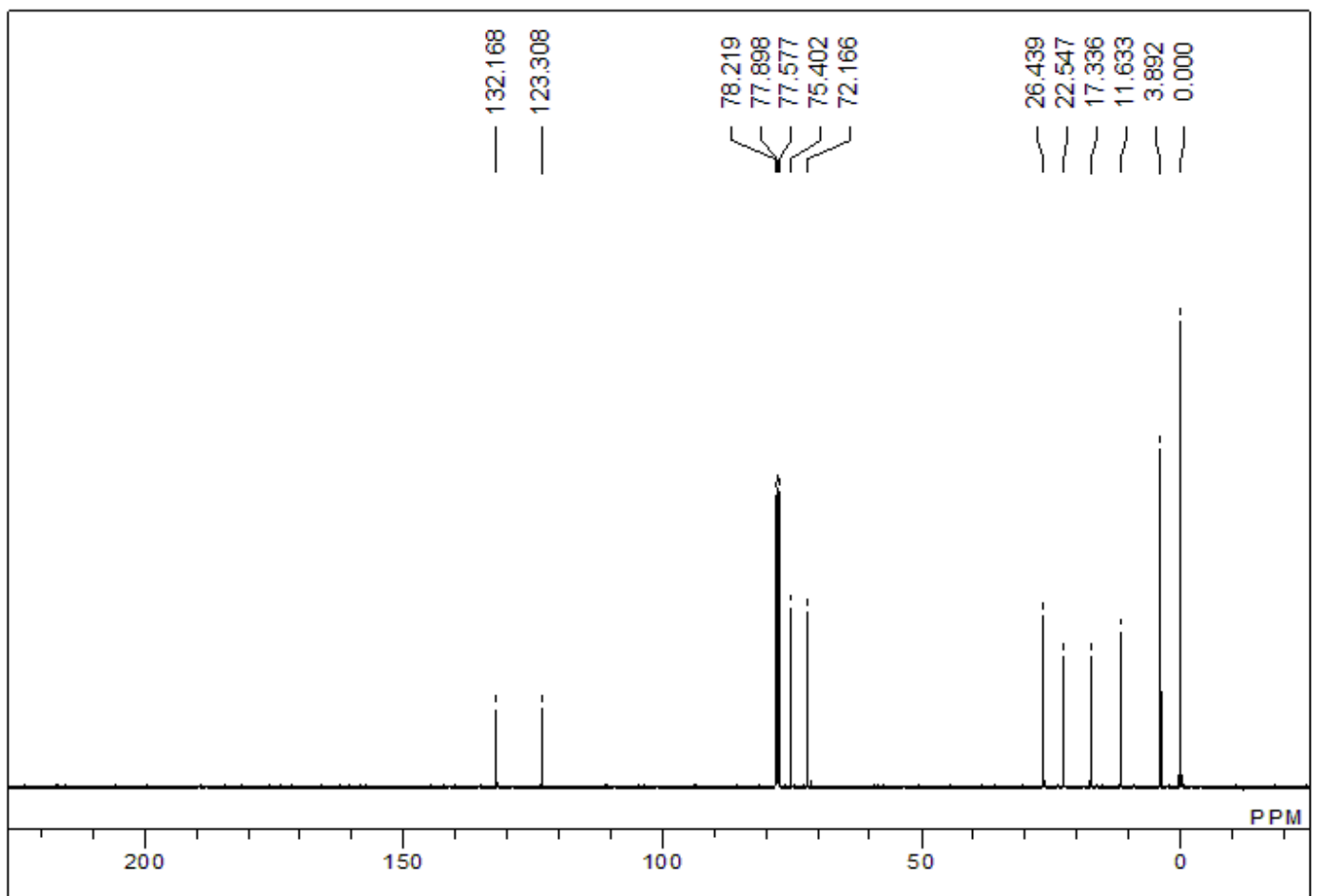


4q $\mathrm{NOE}\left(400 \mathrm{MHz} ; \mathrm{CDCl}_{3}\right)$ :
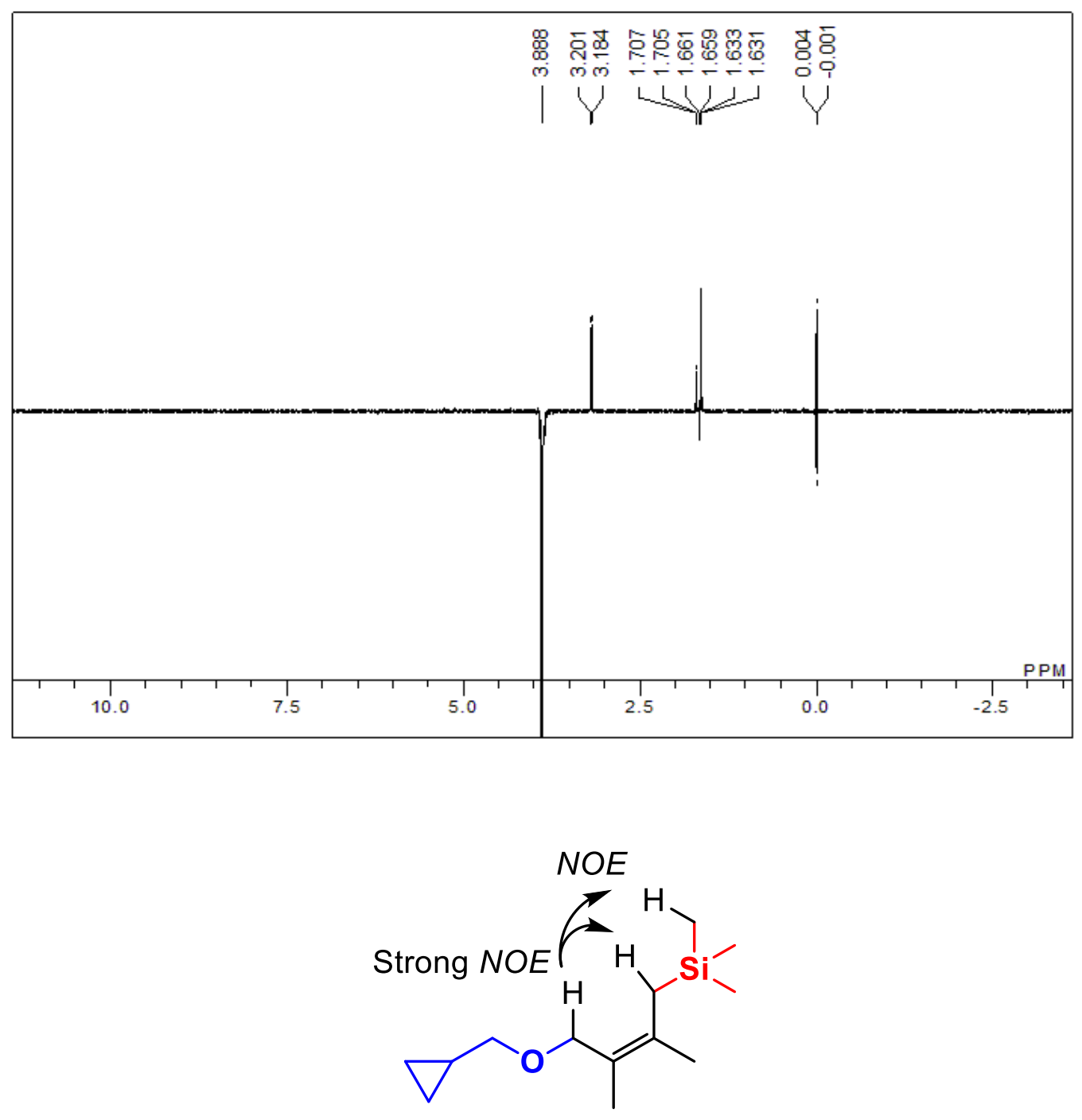

4q: Irradiation at the methylene proton-bearing ether group $(\delta=3.89)$ caused strong enhancement of the methylene proton $(\delta=1.63)$. 
4r ${ }^{1} \mathrm{H}$ NMR (400 MHz; $\left.\mathrm{CDCl}_{3}\right)$ :

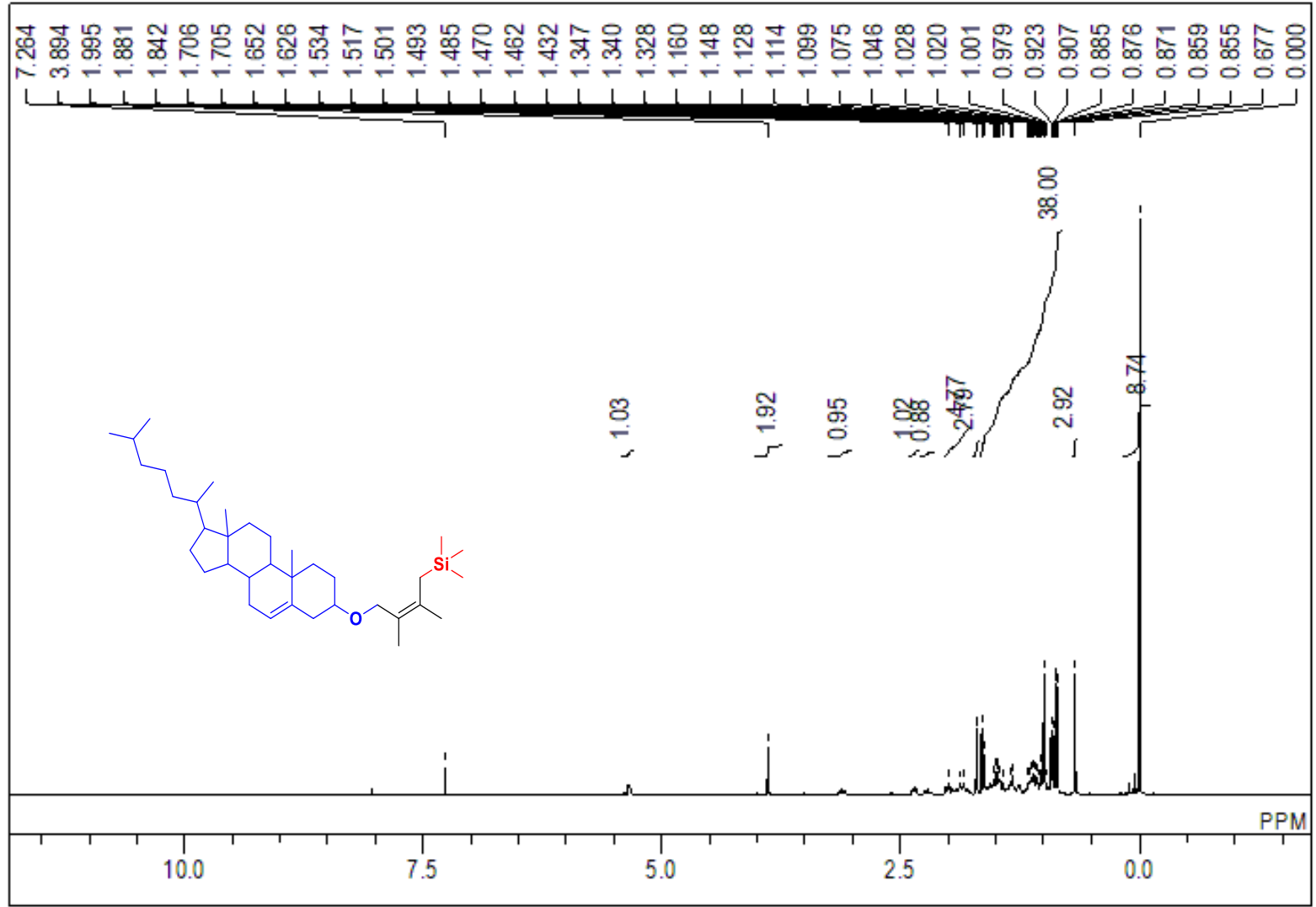

\section{4r ${ }^{13} \mathrm{C}$ NMR (100 MHz; $\left.\mathrm{CDCl}_{3}\right)$ :}

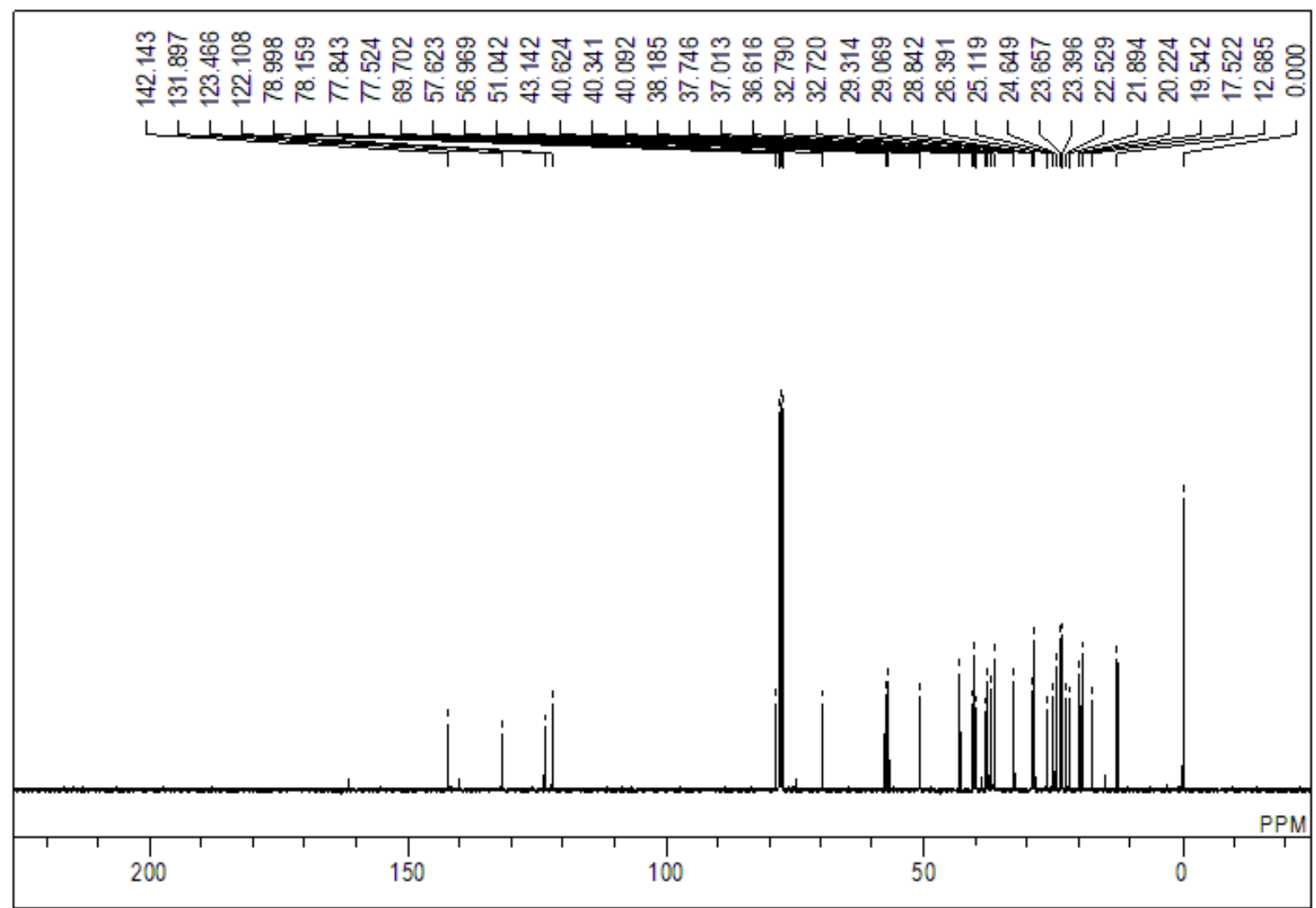


4r NOE (400 MHz; $\left.\mathrm{CDCl}_{3}\right)$ :
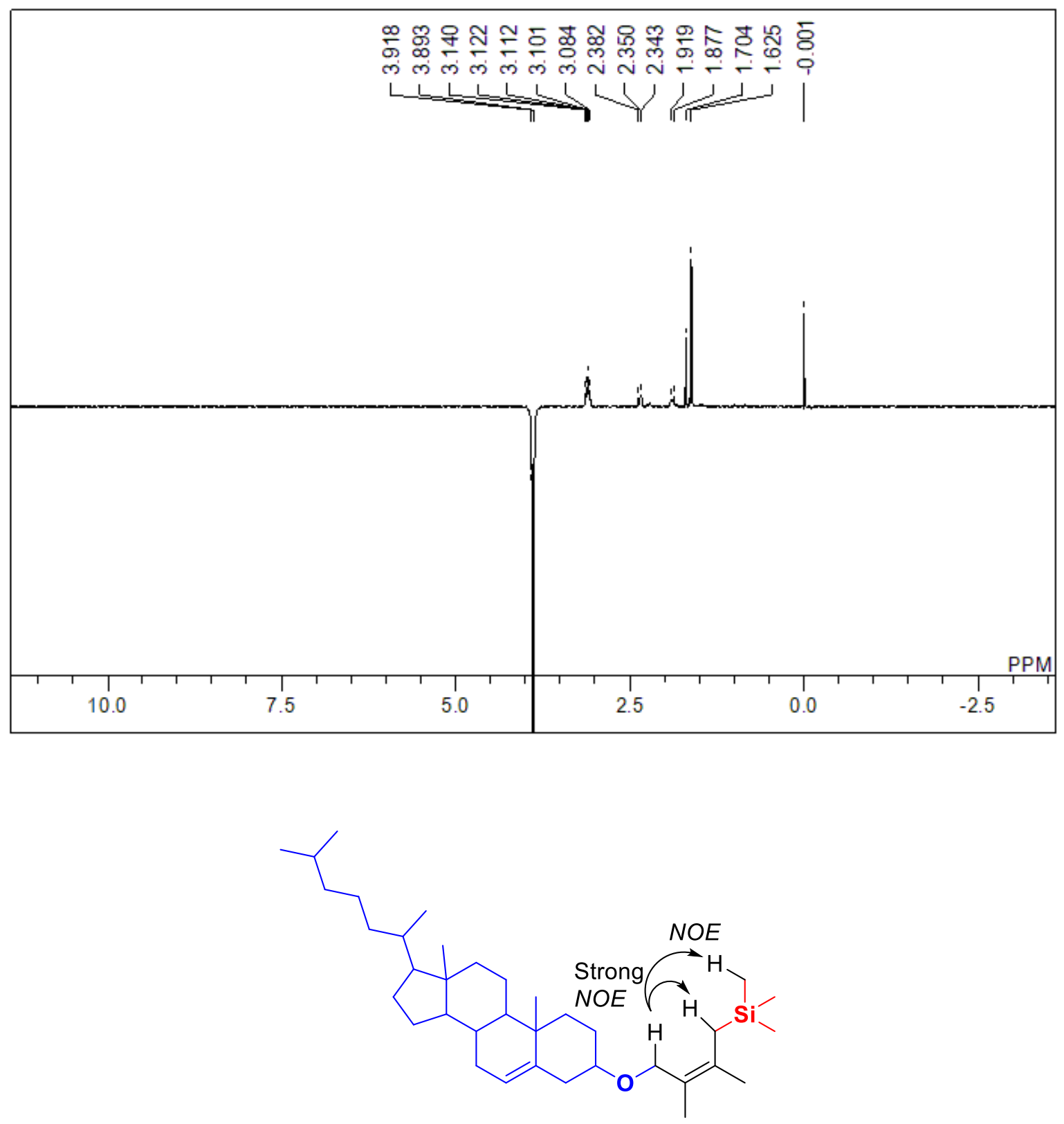

4r: Irradiation at the methylene proton-bearing ether group $(\delta=3.89)$ caused strong enhancement of the methylene proton $(\delta=1.63)$. 
4s ${ }^{1} \mathrm{H}$ NMR (400 MHz; $\mathrm{CDCl}_{3}$ ):

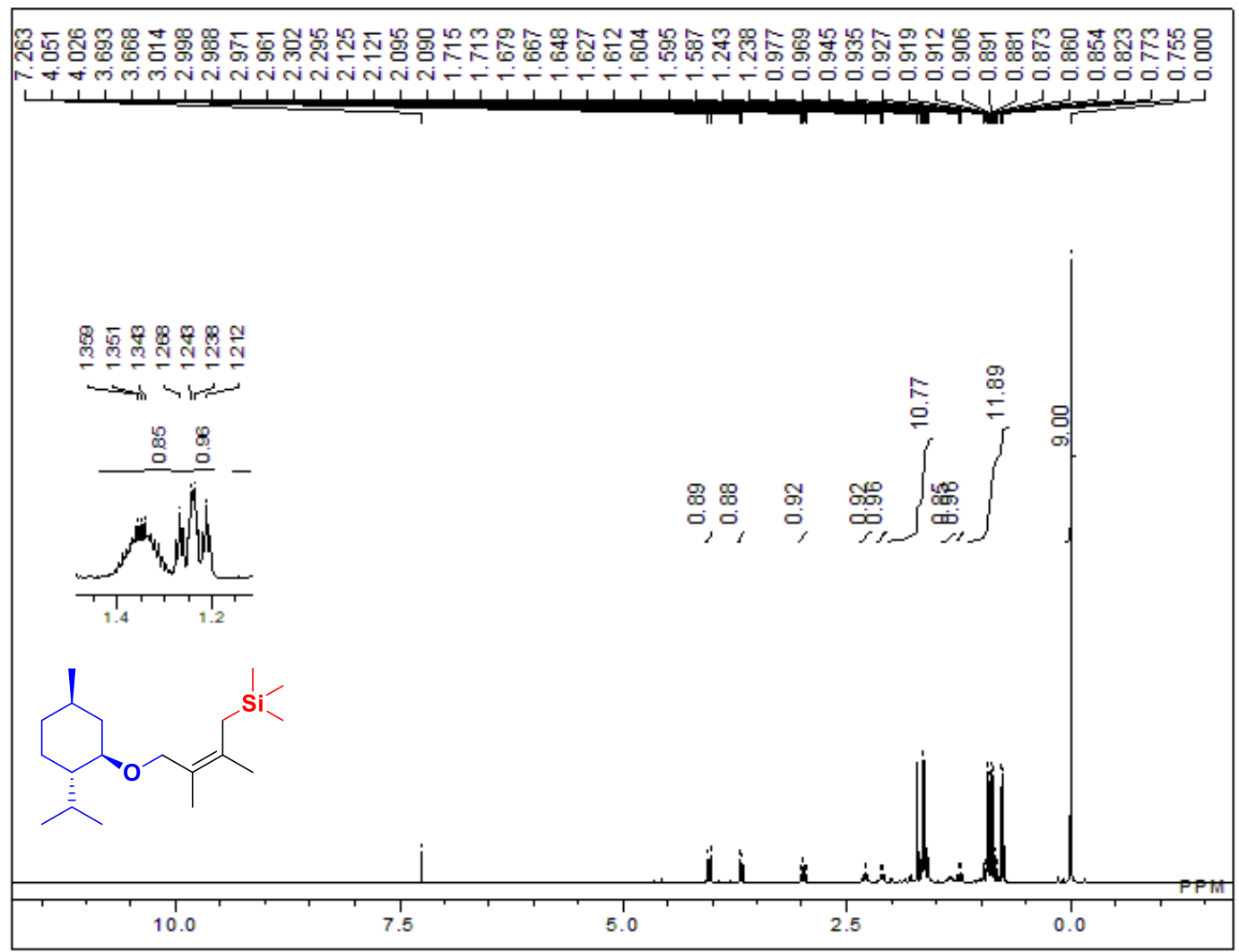

4s ${ }^{13} \mathrm{C}$ NMR (100 MHz; $\left.\mathrm{CDCl}_{3}\right)$ :

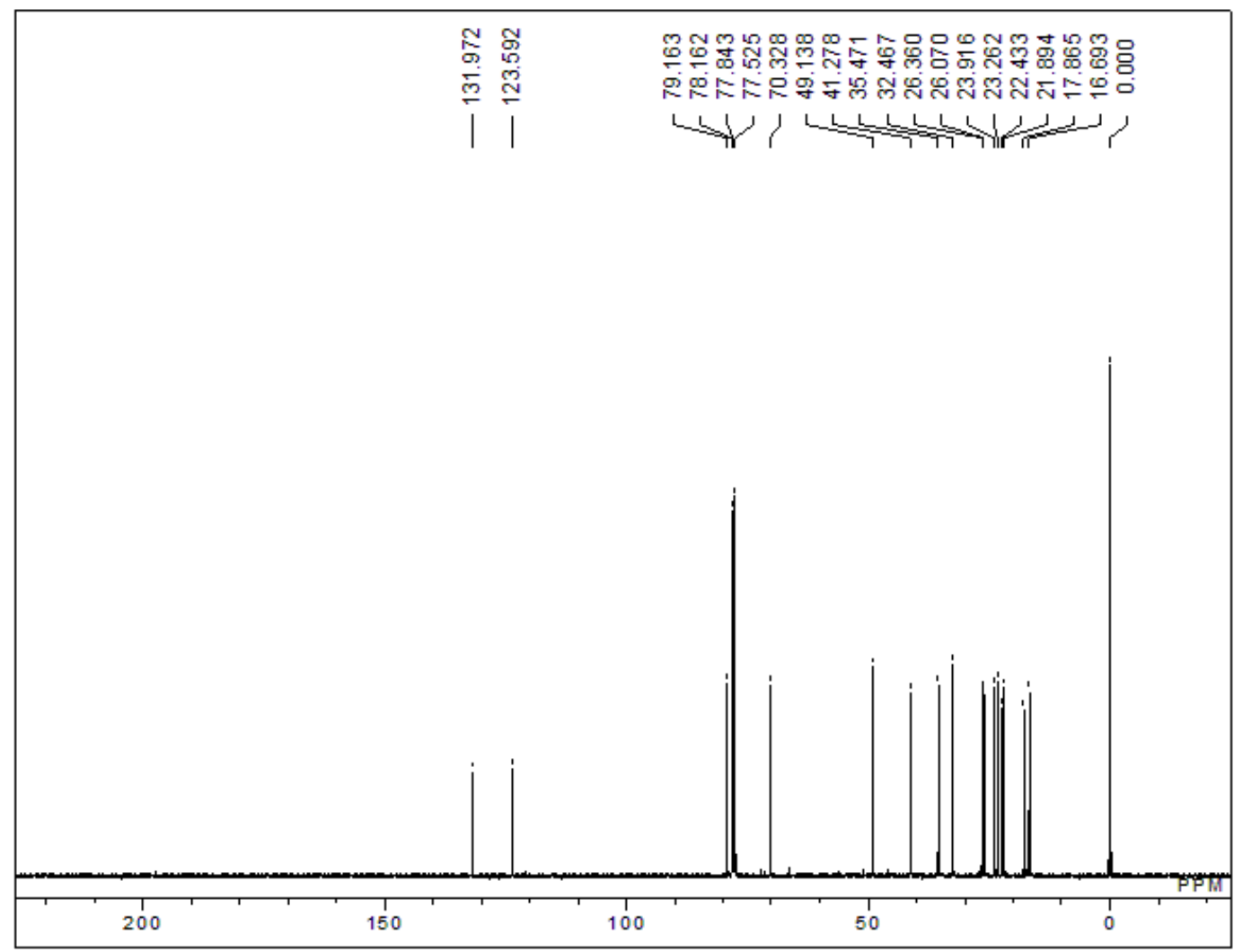


4s $\mathrm{NOE}$ (400 MHz; $\left.\mathrm{CDCl}_{3}\right)$ :
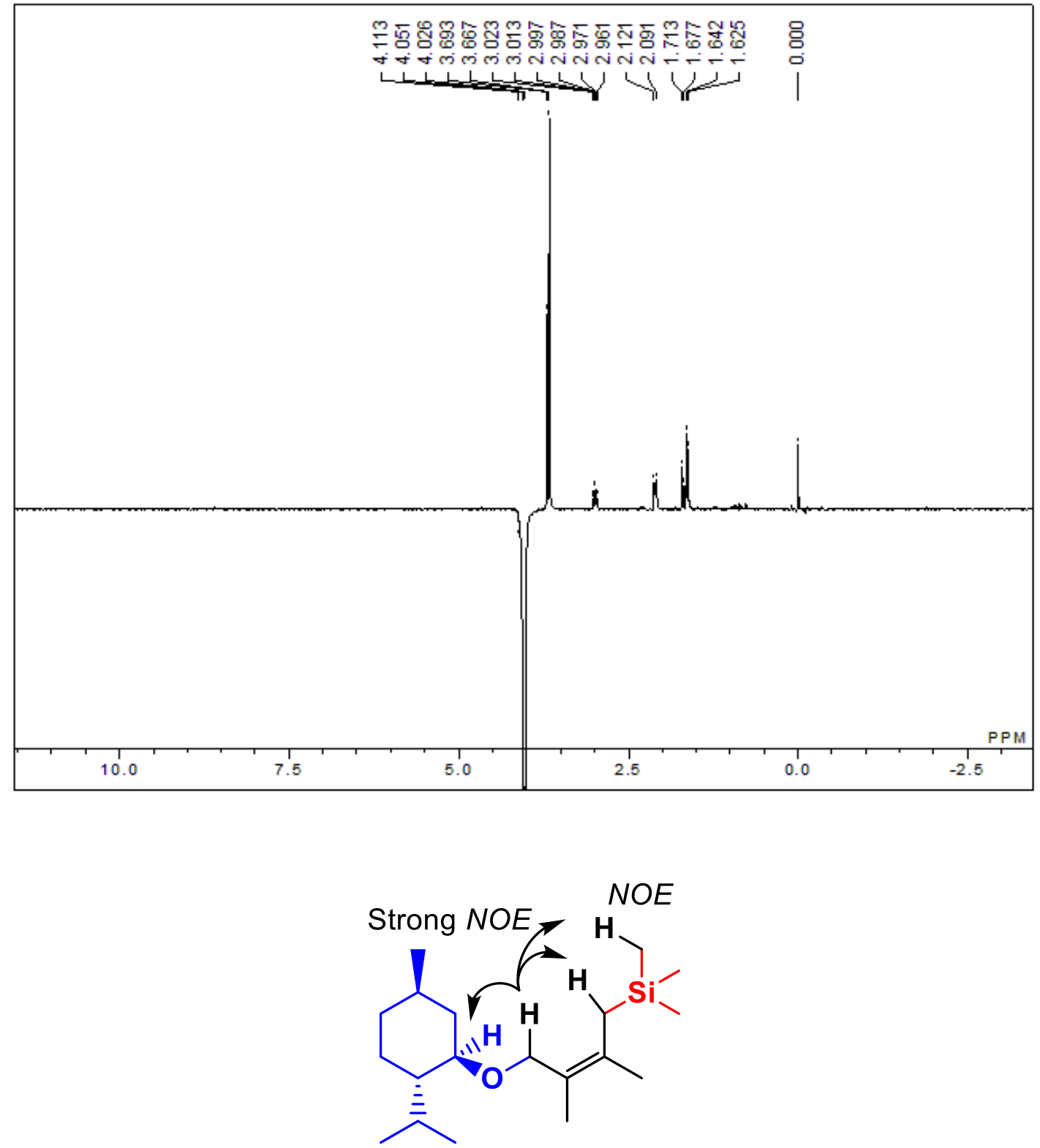

4s: Irradiation at the methylene proton-bearing ether group $(\delta=4.11)$ caused strong enhancement of the methylene proton $(\delta=1.63)$. 
4t ${ }^{1} \mathrm{H}$ NMR (400 MHz; $\mathrm{CDCl}_{3}$ ):

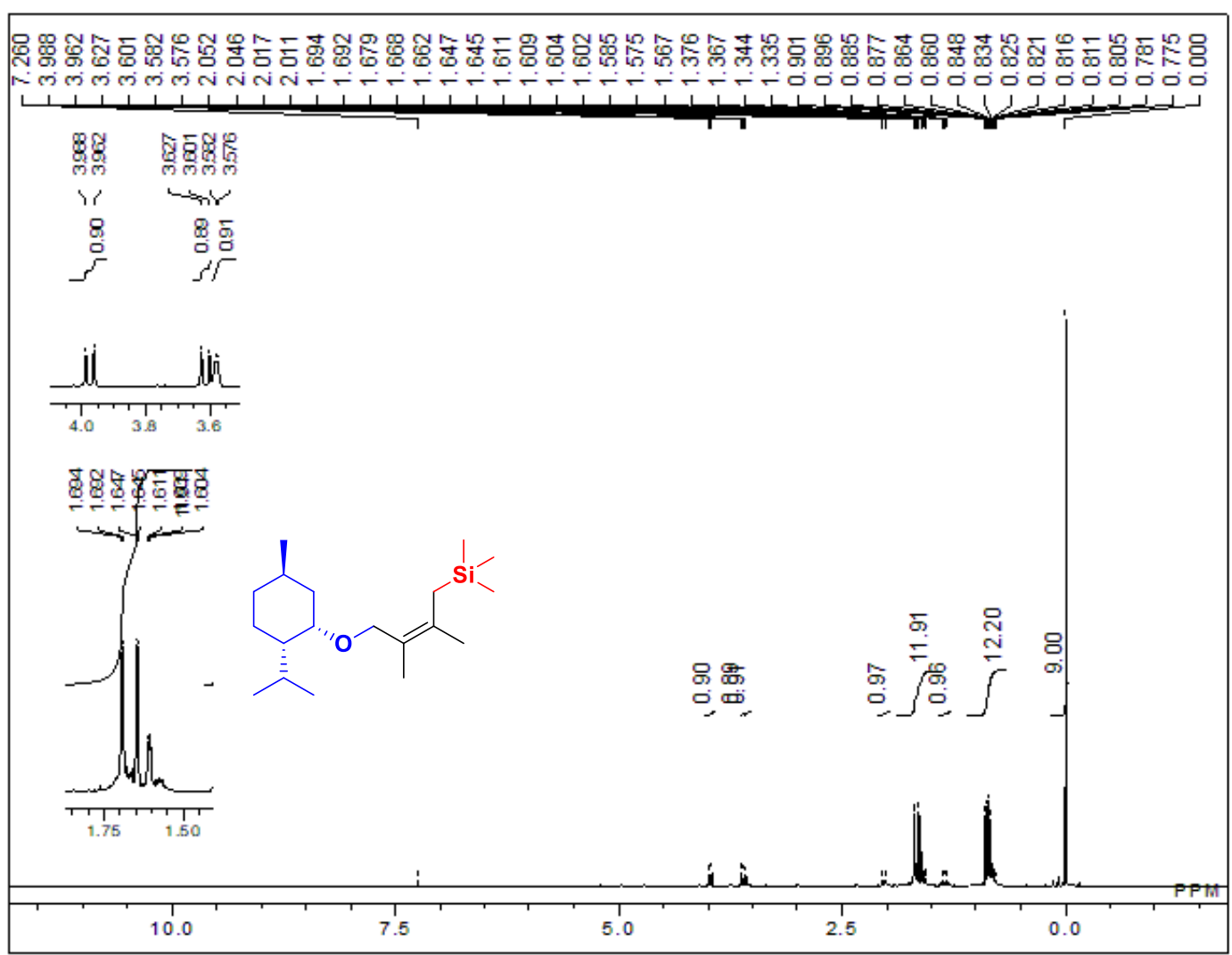

4t ${ }^{13} \mathrm{C}$ NMR (100 MHz; $\left.\mathrm{CDCl}_{3}\right)$ :

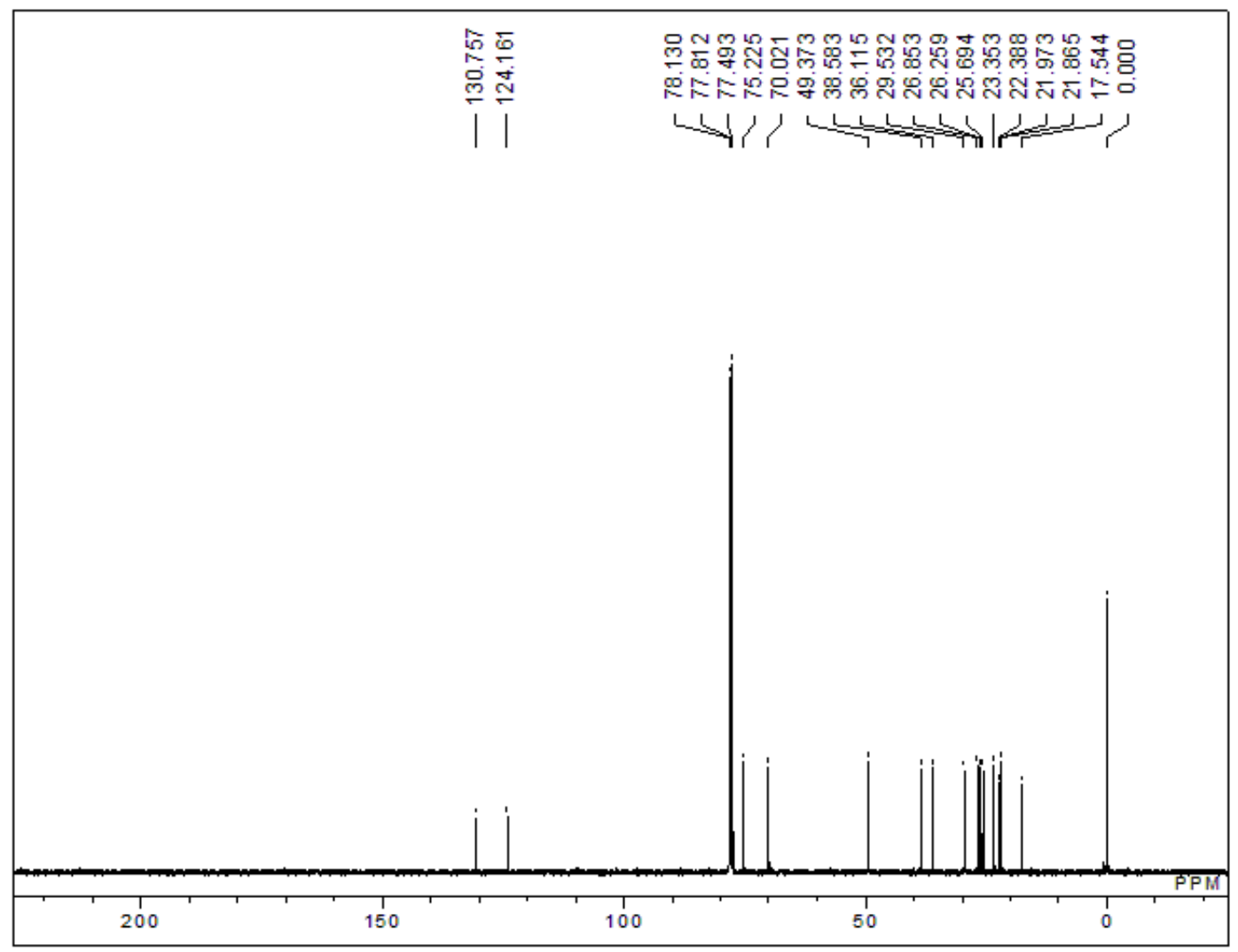


4t $\mathrm{NOE}\left(400 \mathrm{MHz} ; \mathrm{CDCl}_{3}\right)$ :
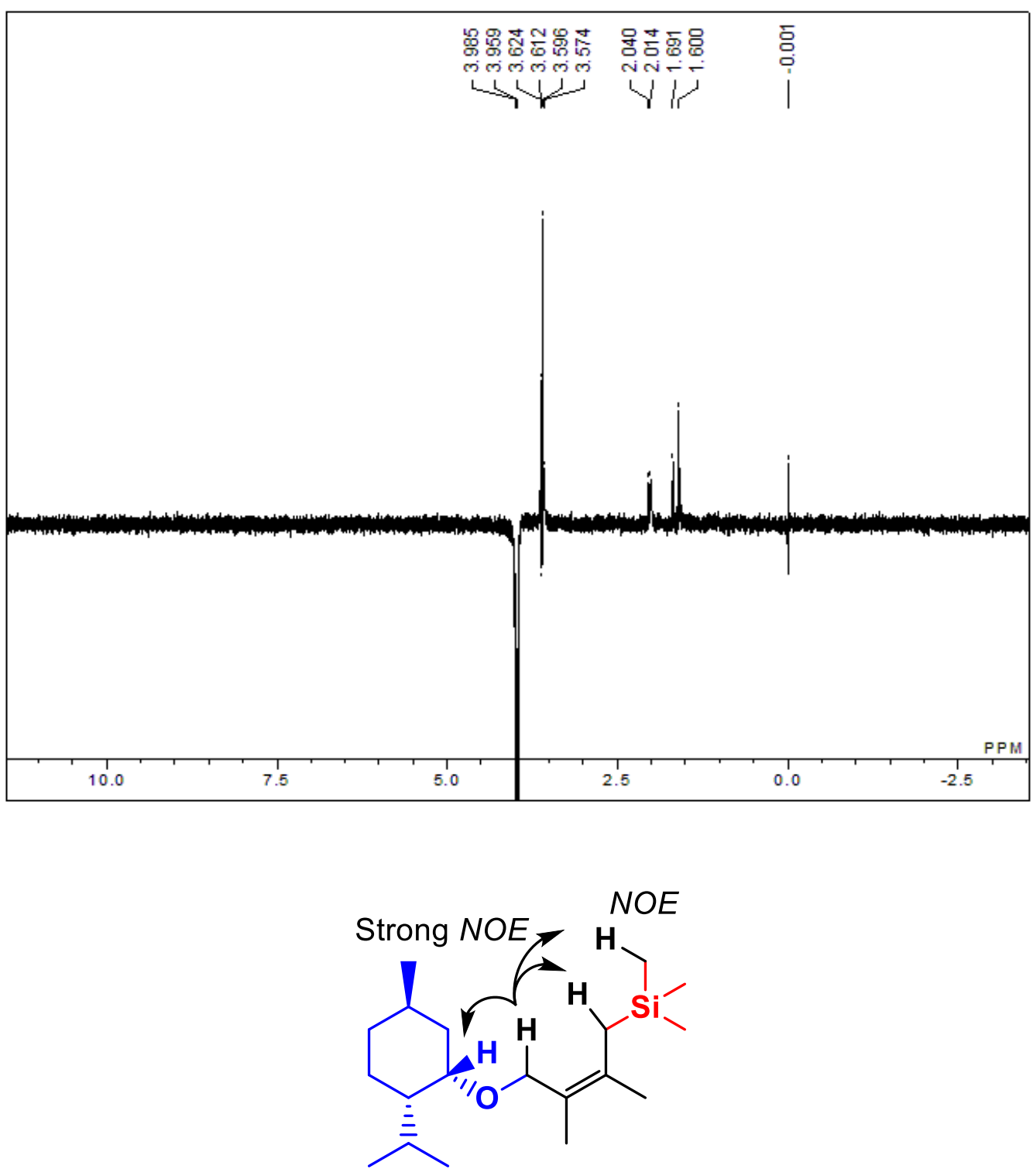

4t: Irradiation at the methylene proton-bearing ether group $(\delta=3.99)$ caused strong enhancement of the methylene proton $(\delta=1.60)$. 
4u ${ }^{1} \mathrm{H}$ NMR (400 MHz; $\left.\mathrm{CDCl}_{3}\right)$ :

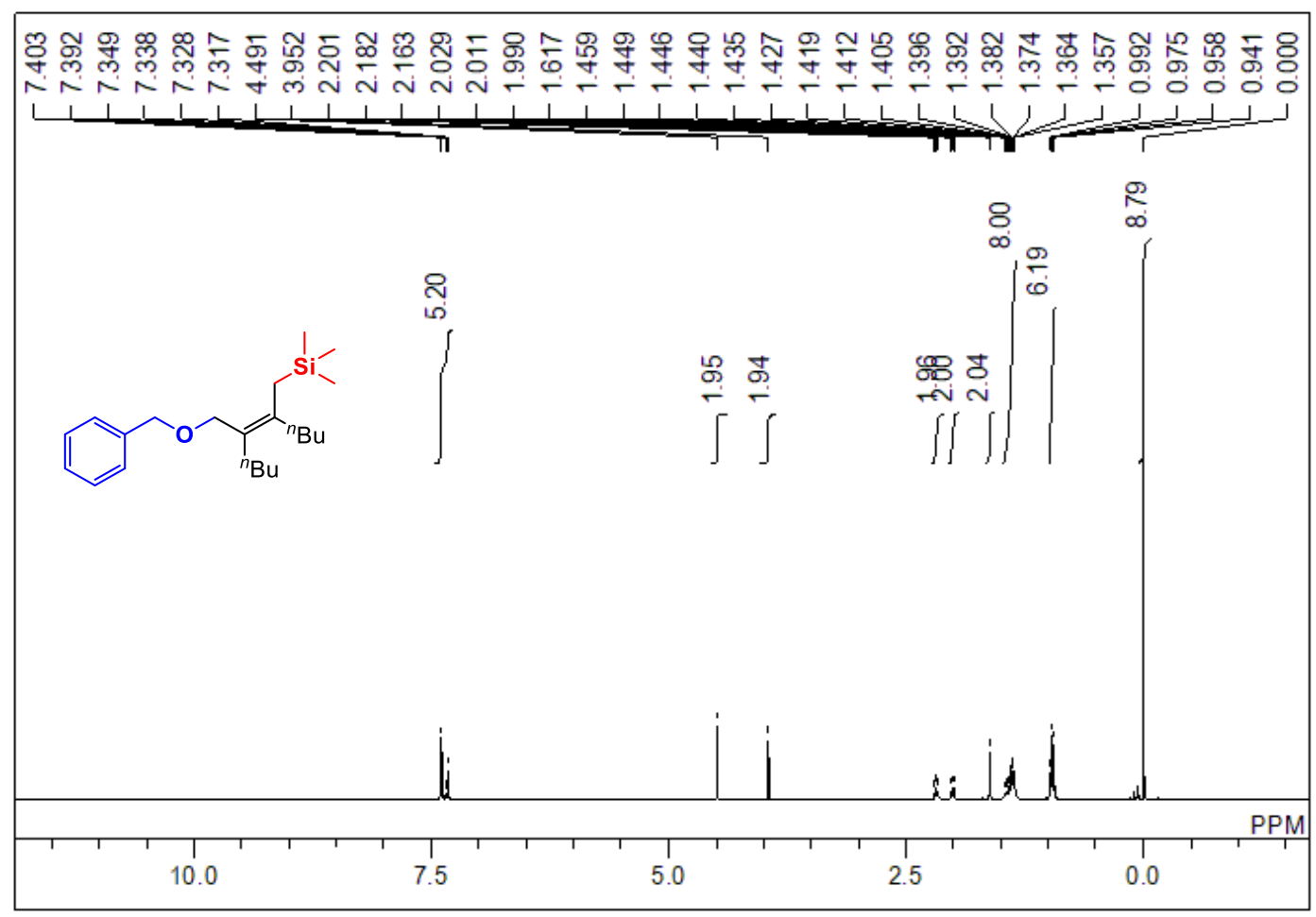

4u ${ }^{13} \mathrm{C}$ NMR (100 MHz; $\left.\mathrm{CDCl}_{3}\right)$ :

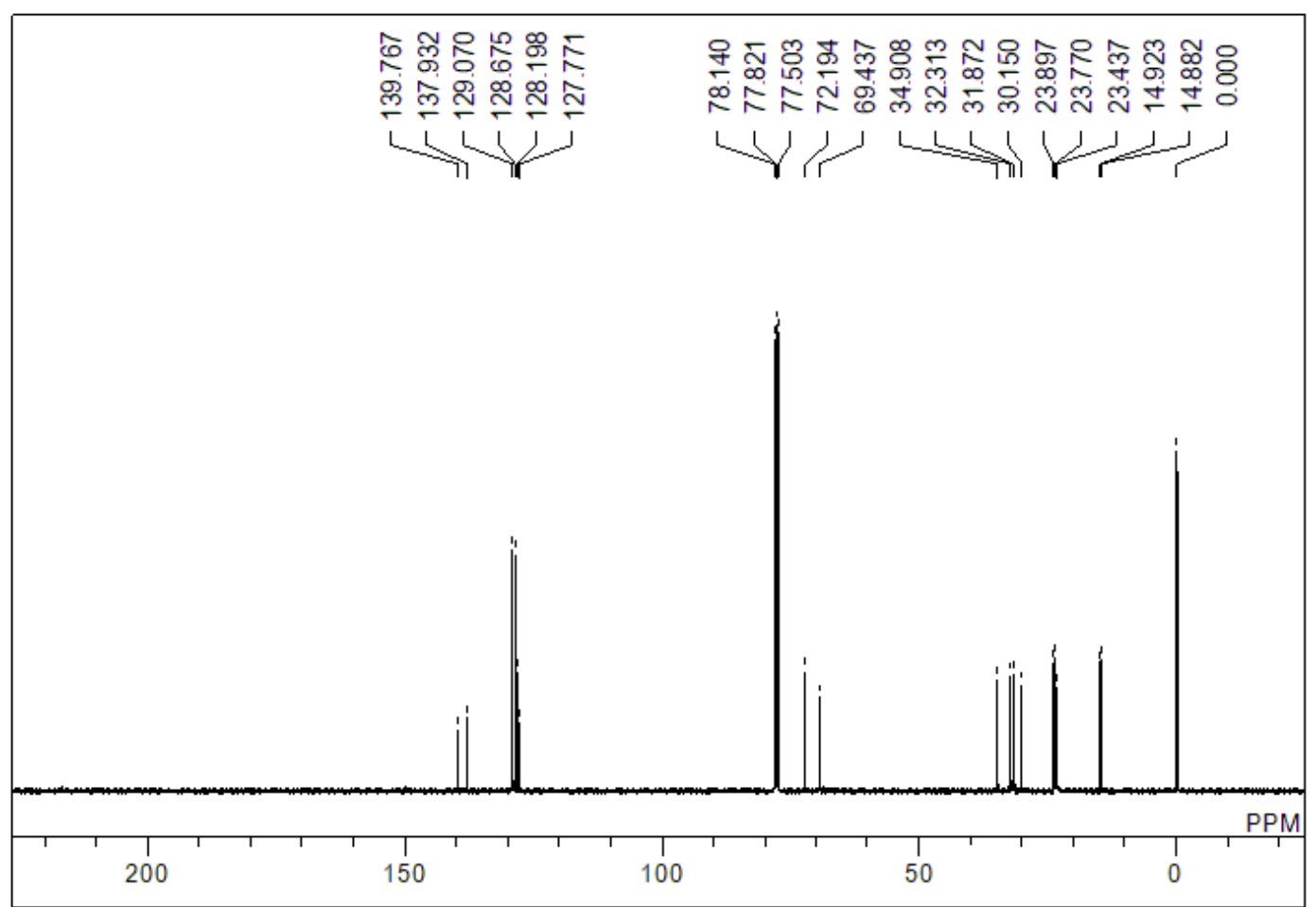


4u NOE (400 MHz; $\left.\mathrm{CDCl}_{3}\right)$ :
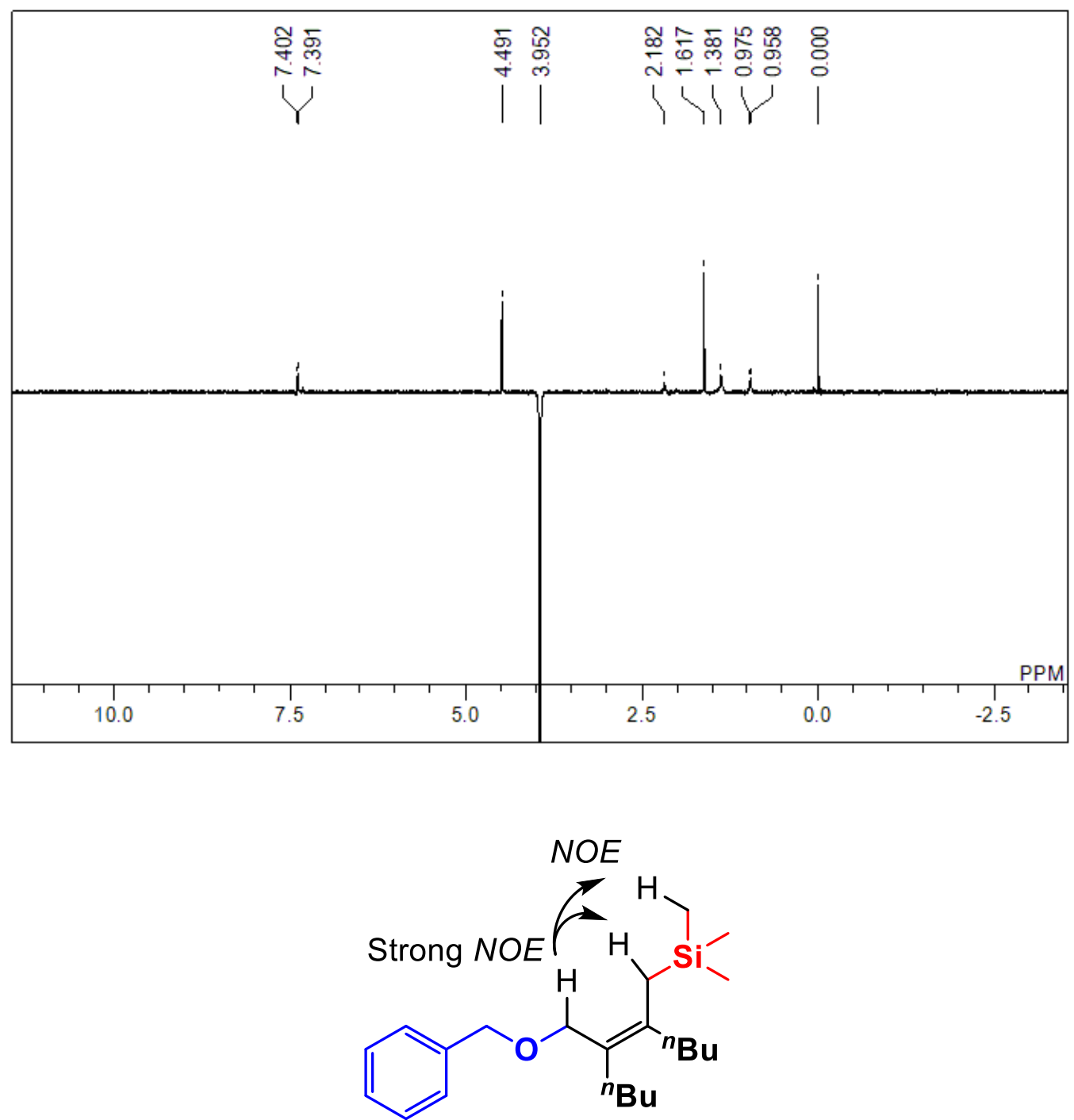

4u: Irradiation at the methylene proton-bearing ether group $(\delta=3.95)$ caused strong enhancement of the methylene proton $(\delta=1.62)$. 